Aus der Klinik für Mund-, Kiefer- und Gesichtschirurgie

(Prof. Dr. med. Dr. med. dent. H. Schliephake)

im Zentrum Zahn-, Mund- und Kieferheilkunde

der Medizinischen Fakultät der Universität Göttingen

\title{
Posttraumatische Reifung \\ und Lebensqualität bei Patienten \\ mit Plattenepithelkarzinomen im Kopf-Hals-Bereich \\ - Eine retrospektive Analyse -
}

\section{INAUGURAL-DISSERTATION}

zur Erlangung des Doktorgrades

für Zahnheilkunde

der Medizinischen Fakultät

der Georg-August-Universität zu Göttingen

vorgelegt von

Johanna Josephine Leonhard

aus Lauterbach

Göttingen 2018 
Dekan:

Referent/in:

Ko-Referent/in:

Drittreferent/in

Datum der mündlichen Prüfung:
Prof. Dr. rer. nat. H. K. Kroemer

PD Dr. Dr. Rudolf Gruber

Prof. Dr. von Nicole Steinbüchel-Rheinwall

Prof. Dr. Thomas Meyer

16.05.2019 
Hiermit erkläre ich, die Dissertation mit dem Titel " Posttraumatische Reifung und Lebensqualität bei Patienten mit Plattenepithelkarzinomen im Kopf-Hals-Bereich -Eine retrospektive Analyse -“ eigenständig angefertigt und keine anderen als die von mir angegebenen Quellen und Hilfsmittel verwendet zu haben.

Göttingen, den

(Unterschrift) 


\section{$\underline{\text { Inhaltsverzeichnis }}$}

$\begin{array}{lll}\text { Abbildungsverzeichnis } & \text { III }\end{array}$

Tabellenverzeichnis $\quad$ IV

Abkürzungsverzeichnis $\quad$ V

1 EINLEITUNG 1

$1.1 \quad$ Begriffserklärung und Definitionen 1

1.2 Psychologische Modelle 5

1.2.1 Ein multifaktorielles Rahmenmodell 7

1.2.2 Modell der Posttraumatischen Reifung 9

1.2.3 Das Janus-Modell 11

1.3 Posttraumatische Reifung 13

1.3.1 Bereiche der PTR 13

$\begin{array}{lll}\text { 1.3.2 Einflussfaktoren } & 14\end{array}$

$\begin{array}{lll}1.4 & \text { Kopf-Hals-Malignome } & 17\end{array}$

$\begin{array}{lll}\text { 1.4.1 Epidemiologie } & 17\end{array}$

$\begin{array}{lll}\text { 1.4.2 TNM-Klassifikation } & 18\end{array}$

$\begin{array}{lll}\text { 1.4.3 Standardtherapie } & 20\end{array}$

2 ZIELSETZUNG 22

3 PATIENTENGUT UND METHODIK 23

$\begin{array}{lll}3.1 & \text { Patientengut } 23\end{array}$

3.2 Studiencharakteristika 23

3.3 Datenerhebung 23

3.4 Fragebögen 25

3.4.1 Fragebogen zur Lebensqualität 25

3.4.2 Fragebogen zur Posttraumatischen Reifung 31

3.5 Statistische Auswertung 33

4 ERGEBNISSE $\quad 34$

4.1 Beschreibung der Stichprobe 34

4.2 Fragebogen zur Persönlichen Posttraumatischen Reifung 34

4.2.1 Einflussfaktor Zeit 34 
4.2.2 Persönliche Beziehungen 37

4.2.3 Neue Möglichkeiten 38

4.2.4 Persönliche Stärke 39

4.2.5 Wertschätzung des Lebens 40

4.2.6 Religiöse Veränderungen 41

4.2.7 Einflussfaktor Geschlecht 42

4.2.8 Einflussfaktor Alter 43

4.2.9 Einflussfaktor Familienstand 44

4.2.10 Einflussfaktor Religion 45

4.3 Fragebogen zur gesundheitsbezogenen Lebensqualität (LQ) 47

4.3.1 Einflussfaktor Zeit 47

4.3.2 Einflussfaktor Geschlecht 50

4.3.3 Einflussfaktor Alter 51

4.3.4 Einflussfaktor Familienstand 52

4.3.5 Einflussfaktor Religion 53

4.3.6 Detaillierte Untersuchung der physischen Funktion 54

4.3.7 Detaillierte Untersuchung der sozial-emotionalen Funktion 56

4.3.8 Einfluss der gewählten Therapie auf die Lebensqualität 58

4.3.9 Auswertung der Wichtigkeitsfragen 59

4.4 Zusammenhang zwischen Posttraumatischer Reifung und Lebensqualität 63

5 DISKUSSION

5.1 Ergebnisse zur Posttraumatischen Reifung 65

$\begin{array}{lll}5.2 & \text { Ergebnisse zur Lebensqualität } & 70\end{array}$

5.3 Zusammenhang zwischen Lebensqualität und Posttraumatischer Reifung 74

$\begin{array}{lll}5.4 & \text { Limitationen der Studie } & 75\end{array}$

$\begin{array}{lll}5.5 & \text { Schlussfolgerungen } & 76\end{array}$

6 ZUSAMMENFASSUNG

7 ANLAGE

$\begin{array}{lll}7.1 & \text { Tabellen } & 79\end{array}$

8 LITERATURVERZEICHNIS 


\section{Abbildungsverzeichnis:}

Abbildung 1: Multifaktorielles Rahmenmodell 7

Abbildung 2: Modell des Prozesses der Posttraumatischen Reifung 9

Abbildung 3: UW-QOL v4-Fragebogen 26

Abbildung 4: PPR-Fragebogen 32

Abbildung 5: Ergebnisse des PPR-Fragebogens 35

Abbildung 6: PPR-Subskala „Persönliche Beziehungen“(PPR1) 37

Abbildung 7: PPR-Subskala „Neue Möglichkeiten“ (PPR2) 38

Abbildung 8: PPR-Subskala „Persönliche Stärke“ (PPR3) 39

Abbildung 9: PPR-Subskala „Wertschätzung des Lebens“(PPR4) 40

Abbildung 10: PPR-Subskala „Religiöse Veränderungen“(PPR5) 41

Abbildung 11: Einfluss des Geschlechts auf die posttraumatische Entwicklung 42

Abbildung 12: Einfluss des Alters auf die posttraumatische Entwicklung 44

Abbildung 13: Einfluss des Familienstands auf die posttraumatische Entwicklung 45

Abbildung 14: Einfluss der Religion auf die posttraumatische Reifung 46

Abbildung 15: Physische und sozial-emotionale Funktion 48

Abbildung 16: Einflussfaktor Geschlecht $\quad 50$

Abbildung 17: Einflussfaktor Alter 51

Abbildung 18: Einflussfaktor Familienstand $\quad 52$

Abbildung 19: Einflussfaktor Religion 53

Abbildung 20: Physische Funktion im zeitlichen Verlauf 54

Abbildung 21: Sozial-emotionale Funktion im zeitlichen Verlauf 57

Abbildung 22: LQ / Tumorgröße, Operationsart und Radiotherapie 59

$\begin{array}{ll}\text { Abbildung 23: Wichtigkeitsfragen } & 60\end{array}$

$\begin{array}{ll}\text { Abbildung 24: Vergleich } & 61\end{array}$

Abbildung 25: Gesundheitsbezogene Lebensqualität $\quad 62$

Abbildung 26: Gesamte Lebensqualität $\quad 62$

$\begin{array}{ll}\text { Abbildung 27: Korrelogramm } & 64\end{array}$ 


\section{Tabellenverzeichnis:}

Tabelle 1: TNM-Klassifikation für Oropharynxkarzinome 19

Tabelle 2: Stadieneinteilung nach der TNM-Klassifikation 20

Tabelle 3: p-Werte für den Faktor „Geschlecht“ 43

Tabelle 4: p-Werte für den Faktor „Alter“ 44

Tabelle 5: p-Werte für den Faktor „Familienstand“ 45

Tabelle 6: p-Werte für den Faktor „Religion“ 46

Tabelle 7: p-Werte der physischen Funktion 49

Tabelle 8: p-Werte der sozial-emotionalen Funktion 49

Tabelle 9: p-Werte Kauen $\quad 55$

Tabelle 10: p-Werte Sprache, Schlucken $\quad 55$

Tabelle 11:p-Werte Geschmack

Tabelle 12: p-Werte Schulter $\quad 57$

Tabelle 13: p-Werte / Zusammenhang PTR und LQ 64

Tabelle 14: Korrelationskoeffizienten $\quad 64$

Tabelle 15: MW und SD der physischen Funktion nach Zeitpunkt 79

Tabelle 16: MW und SD der sozial-emotionalen Funktion nach Zeitpunkt 79 


\section{Abkürzungsverzeichnis}

ICD

LQ

MW

NM

PB

PPR

PPR1

PPR2

PPR3

PPR4

PPR5

PS

PTBS

PTG

PTR

PTSD

QOL

RV

SD

TNM

UICC

UW-QOL v4

WSL
International Statistical Classification of Diseases and

Related Health Problems

Lebensqualität

Mittelwert

Neue Möglichkeiten

Persönliche Beziehungen

Persönliche Posttraumatische Reifung

Persönliche Posttraumtische Reifung Subskala 1:

„Intensivierung persönlicher Beziehungen“

Persönliche Posttraumtische Reifung Subskala 2:

„Neue Möglichkeiten“

Persönliche Posttraumtische Reifung Subskala 3:

„Persönliche Stärke“

Persönliche Posttraumtische Reifung Subskala 4:

„Wertschätzung des Lebens“

Persönliche Posttraumtische Reifung Subskala 5:

„Religiöse Veränderungen“

Persönliche Stärke

Posttraumatische Belastungsstörung

Posttraumatic Growth

Posttraumatische Reifung

Posttraumatic Stress Disorder

Quality of Life

Religiöse Veränderungen

Standardabweichung

Klassifikation von malignen Tumoren

Union Internationale Contre le Cancer

University Washington - Quality of Life version 4

Wertschätzung des Lebens 


\section{EINLEITUNG}

Der Gedanke, dass das Durchleben eines psychischen Traumas für ein Individuum sowohl negative als auch positive Folgen zeitigen kann, ist Hunderte von Jahren alt und weit verbreitet (Tedeschi und Calhoun 2004).

Auch Shakespeare beschäftigte sich bereits mit diesem Thema und erkannte schon damals, dass Leiderfahrungen durchaus eine positive Komponente besitzen können: „...Sweet are the uses of adversity, which, like the toad, ugly and venomous, wears yet a precious jewel in his head..." (Shakespeare 2000, S.12).

Der Text verdeutlicht, dass im Leid ein tieferer Sinn verborgen sein kann, dessen Existenz beziehungsweise Wahrhaftigkeit zunächst unerkannt bleibt. Den Vergleich mit der Kröte wählt Shakespeare deshalb, weil in seiner Zeit der Irrglaube existierte, dass sich im Kopf von Kröten ein Edelstein befindet, der heilende Kräfte besitzt.

Meine persönliche Motivation für diese Arbeit ergibt sich durch ein Interesse an psychologischen Prozessen und den damit verbundenen Entwicklungen.

Ich denke, dass psychisches und physisches Wohlbefinden eng miteinander verknüpft sind und das eine das andere beeinflusst. Patienten mit Kopf-Hals-Malignomen leiden häufig unter einer schlechten Lebensqualität, daher sollten angewandte Therapien und die damit verbundenen Auswirkungen noch weiter untersucht werden. Ein besseres Verständnis der Patienten und ein stärkeres Augenmerk auf das psychische Wohlbefinden halte ich persönlich für sehr sinnvoll. Um den Einstieg in die Konzepte der Posttraumatischen Reifung zu vereinfachen, werden im folgenden Kapitel die Fachbegriffe erklärt.

\subsection{Begriffserklärung und Definitionen}

\section{Psychotraumatologie:}

In Deutschland wurde der Begriff erstmals 1991 in Zusammenhang mit der Gründung des Deutschen Instituts für Psychotraumatologie verwendet.

Parallel hierzu wurde Anfang der 1990er Jahre in Amerika der Begriff der psychotraumatology etabliert und vom Forschungsbereich der traumatology abgegrenzt (Maercker 2009). Während sich die Traumatologie ausschließlich mit den physischen Folgen nach einem Trauma beschäftigt, untersucht die Psychotraumatologie dagegen die posttraumatischen psychologischen Veränderungen. 
Trauma:

In der Klassifikation psychischer Störungen ICD-10 werden im Kapitel F 43 Reaktionen auf schwere Belastungen und Anpassungsstörungen beschrieben (ICD-10-GM 2017, S. 200). Ein Trauma wird als ,ein belastendes Ereignis oder eine Situation außergewöhnlicher Bedrohung oder katastrophenartigem Ausmaßes (kurz oder langhaltend), die bei fast jedem eine tiefe Verzweiflung hervorrufen würde“ beschrieben. Im Zentrum dieser Traumadefinition steht entweder eine Todeskonfrontation oder eine andere extreme Gefahrensituation. Im allgemeinen Sprachgebrauch wird der Begriff viel weiter gefasst.

Unter einem persönlichen Trauma werden zum Beispiel auch enttäuschte Erwartungen oder das Auseinanderbrechen einer Beziehung verstanden (ICD-10-GM 2017, S. 200).

Posttraumatische Belastungsstörung (Abk.: PTBS; engl.: Posttraumatic Stress Disorder, Abk.: PTSD):

Im ICD-(International Statistical Classification of Diseases and Related Health Problems)-System wird die PTBS in die Gruppe der ,Reaktionen auf schwere Belastungen und Anpassungsstörungen" eingeordnet und ist unter Punkt F $43.1 \mathrm{zu}$ finden (ICD-10-GM 2017, S. 200). Als verzögerte oder protrahierte Folgereaktion eines oder mehrerer traumatischer Ereignisse ist sie von der akuten Belastungsreaktion abzugrenzen. Eine PTBS ist durch das ungewollte Wiedererleben von Aspekten des Traumas charakterisiert, durch sogenannte Flashbacks sowie durch Schlafstörungen, emotionale Taubheit und Vermeidungsverhalten. Eine Vielzahl möglicher Traumata kommt als Ursache für die Ausbildung einer PTBS infrage. Untersuchungen haben gezeigt, dass sich eine PTBS besonders häufig infolge von Vergewaltigung, Misshandlung und sexuellem Missbrauch in der Kindheit sowie durch Kriegsteilnahme herausbildet (Maercker 2009).

Posttraumatische Reifung (Abk.: PTR; engl.: Posttraumatic Growth, Abk.: PTG):

Dieses Phänomen beschreibt das Erfahren einer subjektiv empfundenen positiven Veränderung infolge eines traumatischen Ereignisses. Nicht das Ereignis an sich, sondern die Art und Weise des Umgangs mit dem traumatischen Erlebnis sind für die PTR ursächlich (Tedeschi und Calhoun 2004). Bei der PTR handelt sich um eine nicht objektiv messbare positive Veränderung. Synonym dazu wird der Begriff des posttraumatischen Wachstums verwendet, welcher erstmals 1996 in der Literatur erschien (Tedeschi und Calhoun 1996). Verwandte Begriffe sind stressbedingtes Wachstum, wahrgenommener Nutzen oder konfliktbedingtes Wachstum. Die Pioniere im Forschungsfeld der PTR, Tedeschi und Cal- 
houn, beschreiben das Trauma als ein Ereignis mit seismischem Ausmaß, das die fundamentalen Bestandteile der Weltsicht und Lebensziele der Betroffenen erschüttert. Die übergeordneten Glaubens- und Wertsysteme werden infrage gestellt und die individuellen Fähigkeiten, mit Kummer und Leid umzugehen, werden massiv herausgefordert.

Im Rahmen der PTR hat der Ruminationsprozess, der von einer zunächst unwillkürlichen in eine bewusstere Form transformiert wird, eine zentrale Bedeutung. Bei der PTR handelt es sich um einen subjektiven Reifungsprozess, der durch das Ruminieren und die Bereitschaft zur interpersonalen Öffnung ausgelöst wird (Tedeschi und Calhoun 2004).

\section{Rumination:}

Rumination oder Ruminieren ist ein anderer Begriff für Wiederkäuen. Bei diesem kognitiven Verarbeitungsprozess wird das eigene Ich in einen neuen Zusammenhang hineingesetzt und die Dinge aus einem anderen Blickwinkel betrachtet. Der Vorgang des Ruminierens führt zu einer größeren Wertschätzung der alltäglichen Dinge sowie des eigenen Lebens. Umso mehr ein Individuum ruminiert, also das erlebte Trauma verinnerlicht und überdenkt, desto wahrscheinlicher ist ein posttraumatisches Wachstum (Calhoun et al. 2000). Viele Menschen berichten von einem gesteigerten Mitgefühl, verbesserten sozialen Beziehungen, einer erweiterten Selbstwahrnehmung und/oder einer neuen Lebensphilosophie sowie neuen Perspektiven (Connerty und Knott 2013). Dabei ist das Ausmaß des Ruminierens von großer Relevanz. Je intensiver die Intrusion ist, also das In-sich-Kehren und das Überdenken, desto stärker wird die PTR empfunden (Calhoun et al. 2000). Bei der Bewertung solcher Zusammenhänge sollte allerdings berücksichtigt werden, dass die Faktoren objektiv nicht messbar sind.

\section{Resilienz:}

Resilienz leitet sich vom lateinischen Begriff resilire $=$ abprallen ab und beschreibt die Fehlertoleranz eines Systems. Die Resilienzforschung wurde in den 1970er Jahren entwickelt. Zunächst beschäftigten sich die Wissenschaftler mit traumatisierten Kindern, die sich trotz widriger Lebensumstände im Sinne seelischer Gesundheit erfolgreich entwickelten. Bei der Resilienz handelt es sich nicht um eine stabile Persönlichkeitseigenschaft, sondern um dynamische, kontextspezifische, inter- und intraindividuelle variierend verlaufende Adaptionsprozesse (Fooken 2009). Das Konzept der Resilienz wird häufig im Kontext mit der PTR thematisiert. Teilweise werden die Begriffe Resilienz und PTR synonym verwendet. Fooken geht zum besseren Verständnis des Begriffs Resilienz auf seine metaphorischen Bedeutungsinhalte ein. Zunächst betrachtet er die Resilienz als Objektei- 
genschaft eines elastischen Körpers. Dieser reagiert auf mechanisches Einwirken mit einer vorübergehenden Deformation und mit der Rückkehr zur ursprünglichen Form. Man kann resilient auch mit fehlertolerant oder fehlerfreundlich beschreiben. Die Fähigkeit eines Stehaufmännchens ist damit vergleichbar, denn aus beliebiger Lage kann dieses immer wieder seine ursprüngliche Position einnehmen. Analog dazu handelt es sich bei einem psychischen Trauma um einen Störzustand, aus dem der Betroffene wieder in seinen Grundzustand zurückkehrt. Diese Resilienz macht eine Bewältigung aversiver Ereignisse oder Risikolagen wahrscheinlicher (Fooken 2009).

\section{Salutogenese:}

(lat. salus: Unverletztheit, Heil, Glück; griech. genesis: Entstehung) Aaron Antonovsky prägte den Ausdruck der Salutogenese (1987) als komplementären Begriff zur Pathogenese. In seiner Forschung widmete er sich der Fragestellung, welche Faktoren die Ausbildung von Traumafolgestörungen verhindern können. Laut Antonovsky führen aversive Lebensumstände dazu, dass Menschen ihre generalized resistance resources (GRR) mobilisieren, um stressige Situationen zu bewältigen (Antonovsky 1997). $\mathrm{Zu}$ den generalisierten Resistenz-Ressourcen gehören der individuelle Charakter, die Intelligenz, der soziale Rückhalt, das Gefühl der Kontrolle, der materielle Besitz, die kulturelle Stabilität und das Kohärenzgefühl. Beim Letzteren handelt es sich um ein andauerndes, aber dennoch dynamisches Gefühl des Vertrauens (Antonovsky 1997, Almedom 2005).

\section{Lebensqualität (Abk.: LQ; engl.: Quality of Life, Abk.: QOL):}

Bei dem Konstrukt Lebensqualität handelt es sich um ein mehrdimensionales Konzept, welches die subjektive Evaluierung von positiven und negativen Komponenten beinhaltet. Neben den physischen, psychologischen und sozialen Dimensionen spielen auch der materielle Lebensstandard und das spirituelle Wohlbefinden eine Rolle. Zur Erfassung der gesundheitsbezogenen Lebensqualität existieren diverse Fragebögen, die das Konstrukt Lebensqualität in unterschiedlichen Dimensionen erfassen. Der UW-QOL v4-Fragebogen (University Washington-Quality of Life v4-Fragebogen) wurde speziell auf die Anforderungen in Bezug auf die Kopf-Hals-Malignome angefertigt und soll Aufschluss über die Lebensqualität in Abhängigkeit von der angewandten Therapie geben. Die Übersetzung des Fragebogens ins Deutsche erfolgte durch Schliephake und Gruber. Übersetzungen des UW-QOL v4-Fragebogen sind auf der Internetseite www.headandneckcancer.co.uk zugänglich. Rogers et al. (2010) empfehlen eine Aufteilung in die beiden Subskalen 1.: phy- 
sische Funktion und 2.: sozial emotionale Funktion, um eine bessere Validierung zu ermöglichen.

\subsection{Psychologische Modelle}

Im Vorfeld der Konzeptualisierung der PTR kam es mit der Einführung des Konzepts der Salutogenese zu einem prinzipiellen Wandel in der Sichtweise der Medizin. Während die Pathogenese die Gesundheit als Normalzustand qualifiziert, hebt die Salutogenese die dichotome Trennung in gesund und krank auf. Der Mensch befindet sich ständig in einem dynamischen Prozess. Gesundheit ist kein stabiler Zustand, sondern muss immer wieder aktiv vom Organismus hergestellt werden. Der Verlust von Gesundheit wird nicht als pathogenetisch, sondern als ein natürlicher allgegenwärtiger Vorgang charakterisiert. Obwohl die Konzepte Resilienz, Salutogenese und PTR aus unterschiedlichen Forschungskontexten resultieren, weisen die Konstrukte eine gewisse Konvergenz auf.

Das Ziel der drei Forschungsansätze ist darauf ausgerichtet, herauszufinden, welche Eigenschaften bzw. Voraussetzungen gegeben sein müssen, um unter extrem aversiven Bedingungen zu bestehen oder im Falle der PTR sogar eine subjektiv empfundene Reifung zu erleben. Der Begriff Resilienz darf dennoch nicht synonym mit PTR verwendet werden. Vielmehr bildet die Resilienz die Grundvoraussetzung für den nachfolgenden Bewältigungsprozess (Fooken 2009). Seit Beginn der Konzeptualisierung der PTR gibt es viele Kritiker, die vor einer Verharmlosung der Thematik warnen. Die Einteilung der Folgen eines Traumas in positive und negative Ergebnisse (PTR bzw. PTBS) ist möglicherweise zu simpel. Es besteht dahin gehend Konsens, dass traumatische Ereignisse einen seismischen Effekt auslösen und das existente Weltbild des Betroffenen erschüttern können (Janoff-Bulman 2004, Cann et al. 2010). Es kann nicht ausgeschlossen werden, dass die beiden Varianten PTR und PTBS synchron in Erscheinung treten können oder dass aus dem Erleben einer PTBS eine PTR hervorgehen kann (Tedeschi und Calhoun 2004). Die Entstehung einer PTR ist noch nicht vollständig erforscht. Ebenso bleibt bis heute die Antwort auf die Frage offen, warum manche Menschen von einer subjektiv empfundenen Reifung berichten, während andere nach dem Erleben einer vergleichbaren traumatischen Situation unter einer PTBS leiden. Das Phänomen der PTR ist multifaktoriell bedingt (Linley und Joseph 2004).

In dem von Tedeschi und Calhoun verfassten Buch „Handbook of posttraumatic growth; research and practice“ aus dem Jahr 2006 werden neben der Vorstellung der theoretischen 
Modelle auch spezifische Kontexte, unter denen eine PTR auftreten kann, wie eine Krebserkrankung, thematisiert. Die Diagnose Krebs stellt für die meisten Menschen ein traumatisches Ereignis dar, das die Ungewissheit und Unkontrollierbarkeit des Lebens vergegenwärtigt (Wicks und Mitchell 2010). Aufgrund des individuell unterschiedlichen Umgangs mit der Erkrankung variieren die posttraumatischen Entwicklungen. Die Krebsdiagnose kann unter anderem auch Auslöser einer unerwarteten positiven persönlichen Veränderung sein (Connerty und Knott 2013). Das Auftreten positiver posttraumatischer Veränderungen bei einigen, aber nicht bei allen Betroffenen wirft die Frage nach den möglichen Einflussfaktoren für diese Entwicklung auf: Über welche Eigenschaften muss ein Mensch verfügen, um gestärkt aus einem traumatischen Ereignis, wie z. B. der Diagnose Krebs, hervorzugehen? Ein weiterer aktueller wissenschaftlicher Fokus im Bereich der Onkologie betrifft die Untersuchung der Lebensqualität. Zur Optimierung und Individualisierung von modernen Therapiekonzepten ist es sinnvoll, die prä- und postoperative Lebensqualität in Abhängigkeit von der gewählten Therapie zu überprüfen. Bei den Malignomen nehmen die Kopf-Hals-Malignome aufgrund ihrer Häufigkeit und ihrer schlechten Prognose eine besondere Position ein. Trotz zahlreicher Fortschritte in der Diagnostik und Therapie konnte die Fünf-Jahres-Überlebensrate in den letzten 20 Jahren nicht deutlich verbessert werden (Mohr et al. 1994, Lang et al. 2002, Rabbels et al. 2005, Maschmeyer et al. 2011). Eine chirurgische Therapie ist potenziell mit dramatischen ästhetischen und funktionellen Auswirkungen verbunden, die nur partiell durch eine Rekonstruktion gemindert werden können. Daher gehen diese Therapien fast immer mit einer verminderten Lebensqualität einher (Borggreven et al. 2007). Wie bereits auf S. 4 erwähnt, beschäftigt sich die Forschung mit der Abhängigkeit der Lebensqualität von der gewählten Therapie. Zur Messung der Lebensqualität stehen diverse Fragebögen zur Verfügung, welche den funktionellen Status sowie das physische, soziale und emotionale Wohlbefinden des Patienten erfassen sollen. Neben dem intra- und interpersonellen Vergleich der prä- und der postoperativen Verläufe können mögliche Einflussfaktoren, wie Alter, Geschlecht, Konfessionszugehörigkeit und Familienstand des Patienten, untersucht werden.

Im Rahmen von Patientenbefragungen können sowohl die Lebensqualität als auch eine eventuell auftretende PTR erfasst werden. Wissenschaftler vermuten, dass zwischen der Lebensqualität und dem Auftreten einer PTR ein Zusammenhang besteht. Möglicherweise ruminieren Patienten mit einer verschlechterten Lebensqualität in einem höheren Maße und erfahren daher eher eine PTR als Patienten mit einer besseren Lebensqualität (Tomich und Helgeson 2012). 


\subsubsection{Ein multifaktorielles Rahmenmodell}

In der Psychotraumatologie existieren unterschiedliche Modelle zur Genese einer PTBS. Die Modelle stimmen darin überein, dass intrapsychische und neurobiologische Prozesse im Zentrum stehen. Dies gilt auch für das multifaktorielle Rahmenmodell von Maercker, das auf Befunden aus der Epidemiologie und verschiedenen PTBS-Forschungsansätzen beruht. Das Modell (S. 7 Abbildung 1) beinhaltet zahlreiche Einflussfaktoren in unterschiedlichen Kategorien und mehrere mögliche Ergebnisse, darunter PTBS und PTR (Maercker 2009).

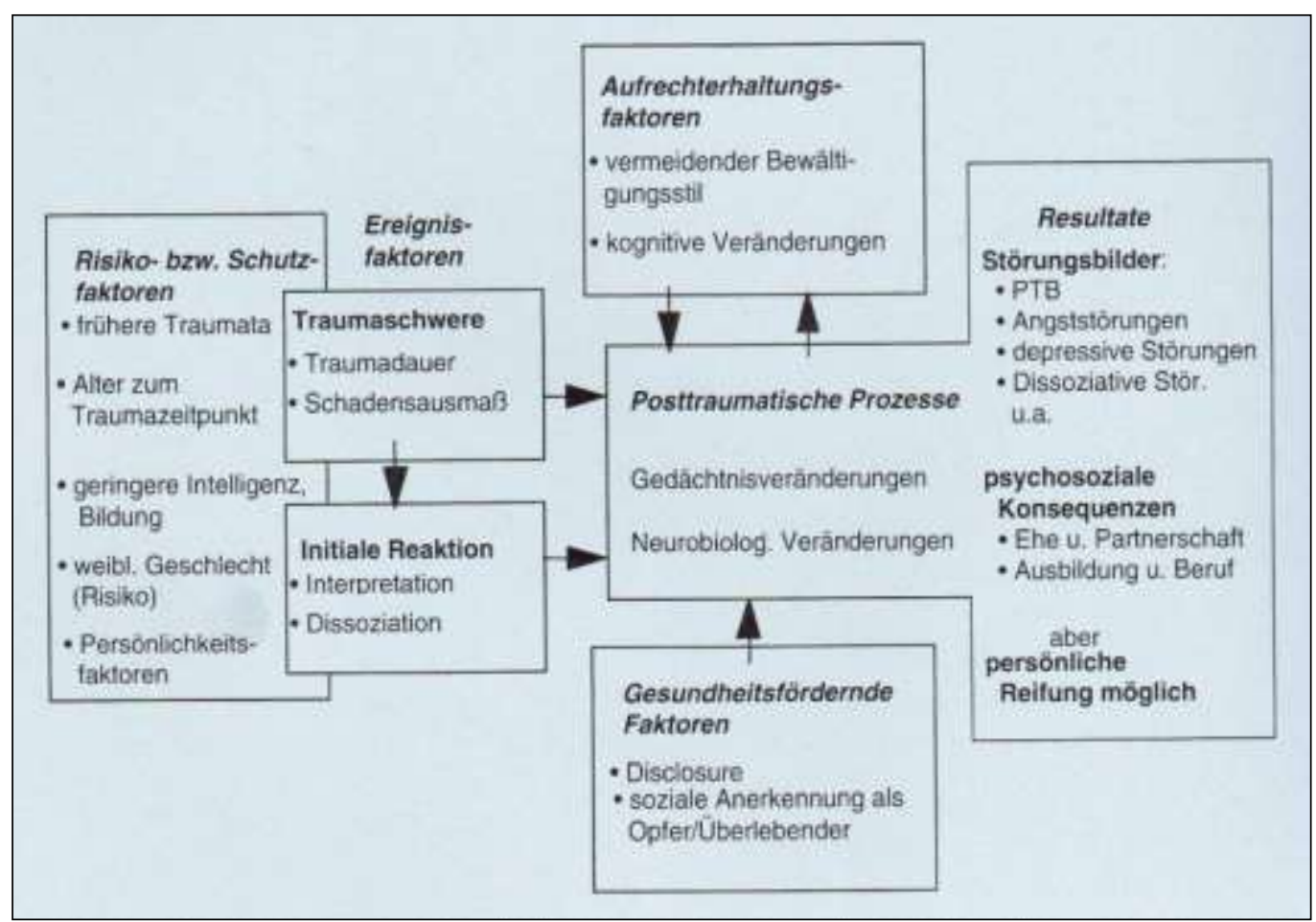

Abbildung 1: Multifaktorielles Rahmenmodell (Maercker 2009, S. 34). Mit freundlicher Erlaubnis von Prof. Maercker, Universität Zürich.

Die Anordnung des Modells lässt eine Einteilung in prä-, peri- und posttraumatische Einflussfaktoren erkennen. Zu den prätraumatischen Risiko- bzw. Schutzfaktoren werden die Persönlichkeitseigenschaften gezählt. Die bisherigen Untersuchungsergebnisse in diesem Bereich sind heterogen und lassen keine signifikanten Korrelationen zwischen spezifischen Persönlichkeitseigenschaften und traumatischem Erleben erkennen (Tempelaar et al. 1989, Calhoun et al. 2000, Tomich und Helgeson 2004, Widows et al. 2005).

Problematisch bei der Untersuchung ist die retrospektive Erhebung der Persönlichkeitseigenschaften vor Einwirkung des Traumas. Ereignisfaktoren, wie Traumaschwere und 
initiale Reaktion, werden als peritraumatische Einflüsse zusammengefasst. Zwischen Traumaschwere und dem Ausmaß der Folgen besteht eine Dosis-Wirkungs-Beziehung (Brewin et al. 2000). Mit Interpretation ist die Einschätzung der Situation gemeint, bei der ein Gefühl der Autonomie als ein positiver Einfluss gewertet wird. Dissoziation beschreibt ein Derealisationsphänomen und bedeutet, dass die betroffene Person durch eine Art Schutzwirkung das Ereignis nicht im vollen Ausmaß realisiert bzw. miterlebt. In diesem Bereich ist die Datenlage allerdings unzureichend.

Die Aufrechterhaltungsfaktoren und gesundheitsfördernden Faktoren gelten als wichtigste Einflusskomponenten. Kognitiv-emotionale Veränderungen führen zu einer anderen Einstellung des Traumaüberlebenden gegenüber seiner Umwelt und sich selbst. Den kognitiven, im Rahmen einer Traumabewältigung stattfindenden Verarbeitungsprozess bezeichnet man auch als Coping. Beim Coping handelt es sich um das Bemühen einer Person, mit der Situationsanforderung und den daraus resultierenden Emotionen umzugehen (Lazarus 2006). Die Rekonstruktion ist Teil des Bewältigungsprozesses und beschreibt den Wiederaufbau des Weltbilds (Janoff-Bulman 2004). Für das Wohlbefinden des Betroffenen ist nicht allein das Trauma wichtig, sondern auch die Art und Weise, wie sein Umfeld damit umgeht. Der Modus und das Ausmaß des Ruminierens beeinflussen das Ergebnis des Bewältigungsprozesses. Durch die Vergegenwärtigung des Erlebten bzw. dessen bewusste Verarbeitung kommt es zu einer veränderten Wertschätzung. Für Überlebende eines Traumas wird das Leben an sich schon deshalb wertvoller, weil es nicht mehr als selbstverständlich angesehen wird. Neben dem kognitiven Verarbeitungsprozess spielt die soziale Unterstützung (gesundheitsfördernde Faktoren) eine große Rolle. Faktoren, wie zwischenmenschliche Einbettung, die Möglichkeit, sich anderen mitzuteilen (Disclosure), und die soziale Anerkennung üben posttraumatisch Einfluss aus. Im Kapitel 4.1 werden die potenziellen Einflussfaktoren detailliert beschrieben. 


\subsubsection{Modell der Posttraumatischen Reifung}

Tedeschi und Calhoun entwickelten ein umfangreiches Modell für den Prozess der PTR.

Eine Variante des Schemas ist im „Handbook of posttraumatic growth; research and practice“ zu finden.

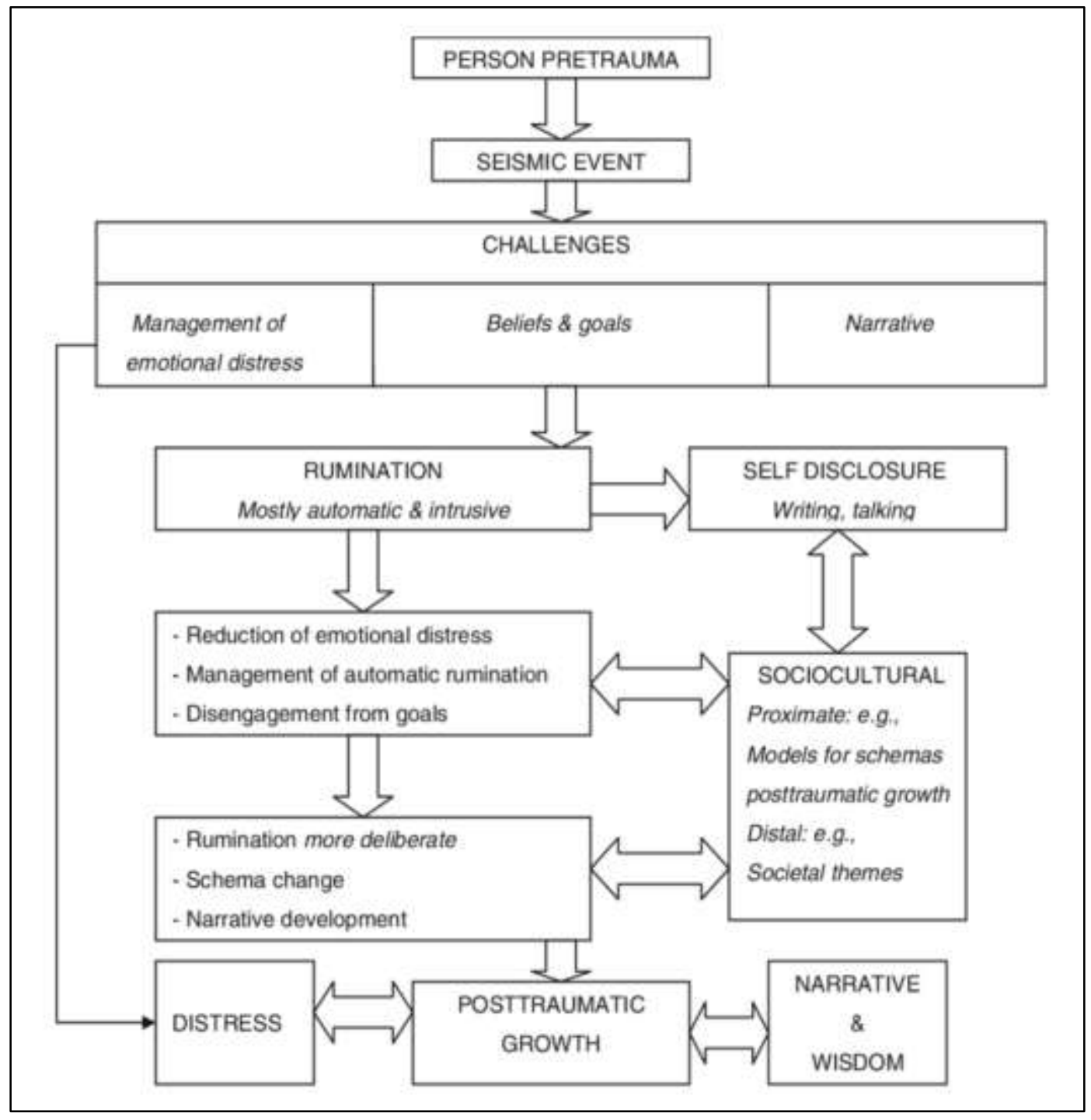

Abbildung 2: Modell des Prozesses der Posttraumatischen Reifung (Calhoun und Tedeschi 2006, S. 8). Mit freundlicher Erlaubnis von Richard G. Tedeschi und Lawrence G. Calhoun.

Wie auf dem Schema in Abbildung 2 zu entnehmen ist, beginnt der Prozess mit der Person vor dem Trauma (Person Pretrauma) und endet mit den positiven Veränderungen, welche sich aufgrund der traumatischen Ereignisse ergeben haben. Die Autoren gehen davon aus, dass nicht der Charakter des Ereignisses, sondern vielmehr die Zerstörung der bisher angenommenen Überzeugung die Transformation initiiert. Dabei muss berücksichtigt werden, dass der kulturelle Hintergrund einen Einfluss auf das angenommene Weltbild nimmt und 
somit auch die Wahrnehmung eines Ereignisses kulturell differiert (Calhoun und Tedeschi 2006).

Diese Unterschiede führen dazu, dass das gleiche Ereignis in unterschiedlicher Weise verarbeitet wird. Ein Ereignis, das bei manchen Menschen das bisherige Weltbild zerstört, kann von anderen Personen in das bisher angenommene Weltbild eingeordnet werden. Janoff-Bulman (2004) formuliert drei Hauptannahmen für das dominierende Weltbild:

- Die Welt ist gütig.

- Die Welt ist bedeutungsvoll.

- Das Selbst ist würdig.

Die meisten Menschen halten die Welt generell für einen positiven Ort, auch wenn sie in manchen Einzelsituationen denken, dass die Welt böse und unfair ist (Janoff-Bulman 2004). Da viele Menschen davon ausgehen, dass die Ereignisse des Lebens eine tiefere Bedeutung haben, versuchen sie zu verstehen, warum bestimmte Vorkommnisse passieren oder warum ein Individuum ein bestimmtes Schicksal erleidet.

Manche Menschen erwarten vom Schicksal Gerechtigkeit. Diese Sichtweise wird dann infrage gestellt, wenn ,gute Mitmenschen“ beispielsweise an Krebs erkranken. Ein solches Schicksal erscheint dann als ungerecht oder sinnlos, was das Weltbild nachhaltig erschüttern kann.

Emotionales Unglück nach einem Trauma kann aber auch dann resultieren, wenn das Geschehnis mit dem angenommenen Weltbild zu vereinen ist. Ein Beispiel ist das Akzeptieren des Todes eines engen Vertrauten als des natürlichen Laufes des Lebens. Emotionales Unglück und PTR schließen einander nicht aus. Das seismische Ereignis leitet den Prozess des Ruminierens ein, der in bestimmten Phasen abläuft und von einem automatischen in einen intrusiven und schließlich in einen bewussteren Prozess übergeht.

Zwischen dem Vorgang des Ruminierens und dem Ausmaß an seelischer Belastung besteht ein Zusammenhang (Calhoun und Tedeschi 2006).

Das automatische Ruminieren ist eng mit der seelischen Belastung und der Zerstörung des bisher angenommenen Weltbilds assoziiert. In der Phase des bewussten Ruminierens übernimmt der Betroffene einen aktiveren Part. Er versucht, Sinn in dem Erlebten zu finden und das erschütterte Weltbild neu aufzubauen. Tedeschi und Calhoun verweisen bei der Frage nach dem Nutzen der PTR auf die Veröffentlichung von Ronnie Janoff-Bulman 2004. Nach dessen Vorstellung entwickeln Personen im Rahmen der PTR eine innere Bereitschaft, die es ihnen ermöglicht, zukünftigen Ereignissen mit weniger Ängstlichkeit gegenüberzutreten, woraus eine geringere Traumatisierung resultiert. 
Janoff-Bulman beschreibt drei Aspekte der PTR (Janoff-Bulman 2004):

1.) Stärke durch Leid

2.) Psychologische Vorbereitung

3.) Existenzielle Reevaluation

Der erste Aspekt thematisiert den vielfach verwendeten Mutmachspruch: "Was uns nicht umbringt, macht uns stärker". Vergleichbar mit dem Muskelaufbau nach physischem Training soll die Bewältigung psychologischer Herausforderungen die persönliche Stärke entwickeln (Janoff-Bulman 2004).

Der zweite Aspekt beschreibt den Vorteil des erfolgreichen Bewältigens. Personen nach einem Trauma sind nicht nur auf mögliche weitere Traumata besser vorbereitet, sondern begegnen diesen mit einem geringeren $\mathrm{Ma} ß$ an Traumatisierung. Tedeschi und Calhoun beschreiben die Auswirkungen von Traumata metaphorisch mit den Folgen eines Erdbebens (Tedeschi und Calhoun 2004). Darauf aufbauend, beschreibt Janoff-Bulman die psychologische Vorbereitung auf weitere Traumata als das Resultat eines physischen Umbauprozesses nach der seismischen Erschütterung. Die zerstörten psychologischen Strukturen werden rekonstruiert und im Zuge des erneuten Aufbaus resistenter und bieten bei folgenden Erschütterungen einen verbesserten Schutz. Der dritte Aspekt beinhaltet die Wiederherstellung der eigenen Weltansicht. Nach dem Durchleben des Traumas versuchen die meisten Betroffenen, einen Sinn in dem Erlebten zu finden (Janoff-Bulman 2004). Mit der Unterstützung ihres Umfelds und kognitiven Bewältigungsmechanismen (Ruminieren) sind Traumaüberlebende dazu in der Lage, ihr Weltbild neu zu ordnen und/oder wiederherzustellen (Calhoun und Tedeschi 2006, Lepore und Revenson 2006).

\subsubsection{Das Janus-Modell}

Aktuell kann nicht abschließend beurteilt werden, ob es sich bei der PTR um eine langfristige positive persönliche Veränderung handelt oder lediglich um eine Form der defensiven Illusion. $\mathrm{Zu}$ dieser Thematik entwickelten Zoellner und Maercker (2006) das JanusModell. Das Modell ist nach dem römischen Gott Janus benannt, auch bekannt als der Gott des Anfangs und des Endes. Er wird mit einem doppelten Gesicht dargestellt und symbolisiert die Dualität aller Dinge und Ereignisse. Das Janus-Modell schlägt vor, dass die PTR sowohl aus einer konstruktiven als auch aus einer illusorischen Komponente besteht, die zeitlich aufeinander folgen. Danach herrscht im Zeitraum nach dem Trauma (proximaler Zeitpunkt) zunächst die illusorische Denkweise vor (Zoellner und Maercker 2006). 
Während dieser Phase überwiegt die selbsttäuschende Komponente, die wiederum mit einem hohen Maß an PTR verbunden ist. Zusätzlich wird diese Phase aber auch durch eine PTBS geprägt. Mit zunehmendem Abstand zum Trauma nehmen die konstruktiven Komponenten der PTR zu, wodurch der Einfluss der PTBS abnimmt. Die Wichtung der Komponenten ist individuell unterschiedlich. Manchen Menschen gelingt durch konstruktives und selbsttranszendiertes Verhalten eine positive psychologische Anpassung, andere verharren dagegen in der Selbsttäuschung. In diesen Fällen ist die subjektiv wahrgenommene PTR eher auf Wunschdenken und Selbstberuhigung zurückzuführen. Manche erklären ihr Schicksal mit: „Wenn es schon passieren musste, muss es doch zu irgendetwas gut sein.“ Die Betroffenen haben so die Möglichkeit, sich nicht mit den negativen Aspekten der Traumaerfahrung auseinandersetzen zu müssen. Dennoch wird auch diese Art der Traumaverarbeitung in einem gewissen Umfang als adaptiv und funktional palliativ und damit als alternativer Weg bewertet (Maercker 2009). Bei der Beurteilung von Bewältigungsprozessen ist die Erfassung des langfristigen Verlaufs der Entwicklungspfade wichtig. Eine tatsächlich stattgefundene persönliche Reifung kann dennoch von begrenzter Dauer sein. Im Laufe des Bewältigungsprozesses kann sich die Gewichtung der Komponenten (konstruktiv/illusorisch) immer wieder verschieben (Zoellner et al. 2008). Die PTR ist nicht zwangsläufig mit dem Wegfall des Auslösers des Traumas verbunden (Zoellner et al. 2008).

Obwohl sich die Begegnung mit einem Trauma bereinigend auf die eigene Sichtweise auswirken kann, bleibt beispielsweise die Auseinandersetzung mit einer lebensbedrohlichen Krankheit, wie Krebs, auch schmerzhaft. Bei einer Krebserkrankung treten neben den seelischen auch körperliche Schmerzen auf. Bedingt durch die Erkrankung und durch die folgenden Therapien, verschlechtert sich die gesundheitsbezogene Lebensqualität. Während Zoellner und Maercker (2006) die PTR eher als einen Teil des Bewältigungsprozesses qualifizieren, bewerten Tedeschi und Calhoun die PTR als mögliches Ergebnis eines Rekonfigurationsprozesses (Lepore und Revenson 2006). 


\subsection{Posttraumatische Reifung}

\subsubsection{Bereiche der PTR}

Leid- und Belastungserfahrungen können potenziell als Quelle für persönliches Wachstum dienen. Bei der PTR handelt es sich nicht um ein Zurückkehren in den prädiagnostischen Zustand, sondern um eine psychologische und emotionale Reifung, die das prätraumatische Niveau übersteigt (Brunet et al. 2010). Das persönliche Wachstum betrifft fünf Bereiche:

1. Wertschätzung des Lebens (Prioritäten)

2. Intensivierung persönlicher Beziehungen (Verbundenheitsgefühl)

3. Entdeckung neuer Möglichkeiten (stärkerer Veränderungswillen)

4. Bewusstwerden eigener Stärken

5. Religiös-spirituelle Veränderungen

(Tedeschi und Calhoun, 1996)

Teil der neuen Lebensphilosophie im Rahmen der PTR ist die veränderte Wahrnehmung dessen, was wirklich wichtig im Leben ist. Auslöser für die veränderte Sichtweise ist häufig eine lebensbedrohliche Erkrankung (Janoff-Bulman 2004). Janoff-Bulman bewertet das Trauma als Herausforderung für das Selbstbild, die Weltsicht und die Zukunftserwartungen der Betroffenen. Das Trauma bewirkt darüber hinaus ein verstärktes Gefühl der eigenen Verletzlichkeit. Die Erkenntnis, dass die Welt unsicher und unberechenbar ist, führt zu einer neuen Wertschätzung des Lebens (Janoff-Bulman 2004).

Das Überstehen des Traumas und das Gefühl, eine zweite Chance zu erhalten, können ein Anlass dafür sein, in seinem Leben neue Prioritäten zu setzen (Tedeschi und Calhoun 1996). Eine bekannte Folge der veränderten Prioritäten ist die verstärkte Wahrnehmung von „kleinen“ Dingen, wie das Lachen eines Kindes. Diejenigen Ziele und Objekte, die vor der Krise wichtig erschienen, verlieren nunmehr ihren Stellenwert, wohingegen andere an Bedeutung gewinnen (Calhoun und Tedeschi 2006, Zoellner et al. 2006).

Auch persönliche Beziehungen erfahren häufig eine Veränderung. Die Menschen, die ein Trauma erlitten haben, berichten von einer Zunahme subjektiv wahrgenommener Nähe und einer Stärkung zwischenmenschlicher Beziehungen (Calhoun und Tedeschi 2006, Lepore und Revenson 2006, Maercker 2009, Zoellner et al. 2006). Darüber hinaus berichten Menschen im Rahmen der PTR von einer ausgeprägten Empfindung der eigenen Stärke. Das Gefühl der Stärke ist oft mit einer vermehrt wahrgenommenen Verletzlichkeit verbunden (Tedeschi und Calhoun 2004). 
Ein weiterer Vorteil der PTR ist laut Janoff-Bulman die psychological preparedness, die eine Zunahme der Resilienz bewirkt. Die Betroffenen sind auf künftige Tragödien besser vorbereitet und können diese mit einer geringeren Traumatisierung überstehen (JanoffBulman 2004). Auch in den existenziellen, spirituellen und religiösen Bereichen wird eine PTR beobachtet. Auch Personen, die nicht religiös sind oder die sich selbst als Atheisten bezeichnen, können eine PTR in dieser Domäne erfahren (Maercker 2009).

\subsubsection{Einflussfaktoren}

Die Frage nach den Einflussfaktoren (Eigenschaften des Betroffenen, externe Faktoren) für eine PTR ist Gegenstand wissenschaftlicher Forschung. Bisher wurden folgende soziodemographische Daten als mögliche Einflussfaktoren diskutiert:

1. Alter, Geschlecht

2. Soziale Unterstützung, Religion

3. Bildungsstand, Einkommen

Weitere mögliche Einflüsse:

4. Resilienz

5. Lebensqualität

6. Ernsthaftigkeit der Erkrankung

Alter und Geschlecht gehören zu denjenigen Faktoren, die möglicherweise Einfluss auf das Ausmaß der PTR nehmen. Die Vergegenwärtigung der Endlichkeit des eigenen Lebens im hohen Alter könnte der Grund für eine geringere PTR sein (Linley und Joseph 2004).

Einige Studien bestätigen die reziproke Korrelation zwischen Alter und dem Ausmaß der PTR (Tempelaar et al. 1989, Klauer 1998, Lechner et al. 2003, Sears et al. 2003, Widows et al. 2005). Bower et al. (2005) nehmen an, dass eine plötzliche und verfrühte Konfrontation mit der Sterblichkeit einen großen Effekt auslöst. Dennoch provoziert die Diagnose Krebs nicht nur die Erkenntnis der eigenen Verletzlichkeit, sondern kann Betroffene auch zu einem Überdenken der eigenen Ansichten und Prioritäten inspirieren.

Die Tatsache, dass Frauen eher zu einer interpersonellen Öffnung und zur Annahme von sozialer Unterstützung bereit sind, wird als Ursache für die größere PTR bei Frauen angenommen (Tempelaar et al. 1989). Die Studienergebnisse zu dieser Thematik sind jedoch nicht homogen (Lechner et al. 2003, Linley und Joseph 2004, Widows et al. 2005). 
Ein anderer wichtiger Einflussfaktor ist das soziale Umfeld der Betroffenen. Unterstützung, Verständnis und Akzeptanz können die PTR fördern (Tedeschi und Calhoun 2004). Personen mit einem sicheren sozialen Umfeld und der Möglichkeit, sich anderen öffnen zu können, werden als resilienter eingestuft. Die soziale Unterstützung erleichtert den Bewältigungsprozess und verringert das emotionale Unwohlsein (Lepore und Revenson 2006). Die Möglichkeit, Gedanken und Ängste aussprechen zu können, eröffnet den Betroffenen die Chance, das Trauma abschließend zu verarbeiten (Lepore et al. 1996). Bei den Personen, die ihre Gedanken unterdrücken bzw. aufgrund des fehlenden sozialen Umfelds nicht die Möglichkeit haben, diese auszudrücken, verlängert sich der kognitive Verarbeitungsprozess und wird als leidvoller empfunden. Die Personen, die in einer Familie bzw. mit einem Partner leben, berichten häufig von wahrgenommener Sicherheit und Rückhalt. Die Studienlage zum Einfluss des sozialen Umfelds auf die PTR ist nicht eindeutig.

In einer Studie korrelierte das Ausmaß des sozialen Rückhalts negativ mit dem Auftreten positiver posttraumatischer Veränderungen (Schmidt et al. 2012). In einer anderen Studie mit Brustkrebspatienten übte die soziale Unterstützung dagegen einen positiven Einfluss aus. Dabei scheint das Sprechen über die Krankheit ein wichtiger Teil der Adaption zu sein (Cordova et al. 2001a).

Weitere wichtige Einflussgrößen für die PTR sind die Kultur- und Religionszugehörigkeit, da sie das Weltbild entscheidend prägen. In den meisten Religionen wird dem Umgang mit Schmerz bzw. Leid eine gewisse Sinnhaftigkeit zugeschrieben (Tedeschi und Calhoun 2004). Der Glaube oder eine philosophische Orientierung kann dazu beitragen, Erlebtes besser zu akzeptieren (Lepore et al. 1996). Studien weisen auf eine positive Assoziation zwischen Religiosität und PTR hin (Linley und Joseph 2004).

Dagegen ist die Datenlage zum Einfluss des Bildungsstands auf die Genese und das Ausmaß der PTR uneinheitlich. Es gibt sowohl Studien, die belegen, dass keine Relation zwischen Bildungsstand und PTR besteht (Lechner et al. 2003), als auch solche, die eine positive Korrelation zwischen Bildungsstand und PTR bescheinigen (Cordova et al. 2001b, Sears et al. 2003). In einer weiteren Studie korrelierte dagegen ein niedriger Bildungsstand mit einem hohen Maß an PTR (Widows et al. 2005). Helgeson et al. (2006) untersuchten den Zusammenhang zwischen soziodemografischen Faktoren und der PTR in einer Studie mit 364 Brustkrebspatientinnen. In dieser Studie berichteten Frauen mit niedrigem Bildungsstand und geringem Einkommen häufiger von einer PTR. 
Eine mögliche Erklärung für diesen Befund ist, dass Frauen mit einem niedrigen sozioökonomische Status routinemäßig mit schwierigen Lebensumständen umgehen müssen und daher über mehr Erfahrung dahin gehend verfügen, negativen Lebensumständen etwas Positives abzugewinnen (Tomich und Helgeson 2004).

Neben den soziodemografischen Parametern kann auch die Personalität ein möglicher Einflussfaktor auf die PTR sein.

Im Zusammenhang mit der Personalität wird häufig die Eigenschaft "Resilienz" (Kapitel 1.1) diskutiert, die als Grundvoraussetzung für die Bewätigung aversiver Lebensumstände gilt (Linley und Joseph 2004). Auch die Lebensqualität stellt einen möglichen Einflussfaktor auf die PTR dar. Patienten mit einer verschlechterten Lebensqualität ruminieren in einem höheren Maße und erfahren somit eher eine PTR als Patienten mit einer besseren Lebensqualität. Wissenschaftler nehmen an, dass ein größerer Leidensdruck mit einem höheren Maß an PTR korreliert (Tomich und Helgeson 2012).

Brustkrebspatientinnen mit einer hohen Lebensqualität berichteten entweder von einem hohen oder von einem niedrigen Maß an PTR, bei einer niedrigen Lebensqualität wurde die PTR dagegen überwiegend als moderat empfunden (Lechner et al. 2006). Die Studienergebnisse zu dieser Thematik sind jedoch nicht eindeutig (Tomich und Helgeson 2012, Helgeson et al. 2006).

Fromm et al. (1996) gehen davon aus, dass das Ausmaß der positiven Traumafolgen mit der Ernsthaftigkeit der Erkrankung korreliert. Eine schlechtere Diagnose macht es wahrscheinlicher, dass der Patient sein Überleben würdigt. Möglicherweise glaubt der Betroffene, aus einem bestimmten Grund überlebt zu haben, und sieht daher eine größere Bedeutung in seinem Schicksal.

Bower et al. (2005) stellen die Hypothese auf, dass die Wahrnehmung der eigenen Verletzlichkeit infolge einer Krebsdiagnose mit einer verstärkten Wahrnehmung der Bedeutung der eigenen Existenz einhergeht. Daher führen möglicherweise Faktoren, die das zerrüttende Erlebnis der Krebserfahrung verstärken, zwar zu einer erhöhten Verletzlichkeit, letztlich aber auch zu einer größeren Wertschätzung des Lebens und zu neuen Prioritäten (JanoffBulman 2004).

Im Folgenden werden die Malignome des hier untersuchten Patientenguts kurz dargestellt. 


\subsection{Kopf-Hals-Malignome}

\subsubsection{Epidemiologie}

$\mathrm{Zu}$ den Kopf-Hals-Malignomen zählen bösartige Tumoren der Mundhöhle, des Naso-, Oro- und Hypopharynx sowie des Larynx, der Nasennebenhöhlen und der Speicheldrüsen. Die malignen Tumoren der Kopf-Hals-Region rangieren nach internationalen Statistiken mit etwa 8\% auf dem sechsten Platz in der Häufigkeit aller Malignome (Wittekind et al. 2001). Weltweit werden nach Schätzungen ungefähr 500.000 neue Fälle pro Jahr diagnostiziert (Thurnher und Heiduschka 2011). Das Plattenepithelkarzinom ist mit 90\% der häufigste histologische Typ unter den Kopf-Hals-Malignomen, wobei die meisten dieser Tumoren im Bereich der Mundhöhle und des Oropharynx lokalisiert sind (Bose et al. 2013). Die öffentliche Wahrnehmung der Problematik ist gering, obwohl ein weltweiter Anstieg der Erkrankungshäufigkeit zu konstatieren ist. In Deutschland erkranken jährlich ca. 13.000 Menschen an Tumoren im Mundhöhlen- und Rachenbereich. Die Gesellschaft der epidemiologischen Krebsregister in Deutschland e.V. gibt für das Jahr 2009 eine Inzidenz von 19,5/100.000 Einwohnern für Männer und von 5,7/100.000 Einwohnern für Frauen an. Männer sind damit wesentlich häufiger betroffen als Frauen; bei Männern steht die Erkrankung an siebter Stelle aller bösartigen Tumoren (S3-Leitlinie Mundhöhlenkarzinom 2012). Das mittlere Erkrankungsalter beträgt bei Männern 60 Jahre und bei Frauen 63 Jahre. Die Mortalität lag im Jahr 2009 laut Krebsregister bei etwas weniger als 50\%.

Hauptrisikofaktoren für die Genese von Plattenepithelkarzinomen im Bereich der Mundhöhle ist ein chronischer Alkohol- oder Nikotinkonsum. Zwischen beiden Risikofaktoren besteht ein multiplikativer Zusammenhang, da Alkohol die Durchlässigkeit des Epithels für Nitrosamine und aromatische Kohlenwasserstoffe erhöht. Eine kontinuierliche Exposition mit diesen Substanzen führt zu Veränderungen der Mukosa im oberen Aerodigestivtrakt. Während viele im Tabak enthaltene Stoffe karzinogen sind und hier eine DosisWirkungs-Beziehung besteht, fungiert der Alkohol selbst nicht als Karzinogen. Alkohol und Tabak wirken also synergistisch (Reichert et al. 2002). Das Erkrankungsrisiko liegt bei gleichzeitigem chronischem Tabak- und Alkoholabusus bis zu einem Faktor 30 höher. Kautabak gehört ebenfalls zu den prädisponierenden Faktoren für die Entstehung eines Plattenepithelkarzinoms in der Mundhöhle (Altieri et al. 2002). Europaweit ist die Inzidenz sowohl bei Männern als auch bei Frauen in Frankreich am höchsten und in Griechenland am niedrigsten. Die Tatsache, dass es keine Unterschiede zwischen dem Tabak- und dem Nikotinkonsum in beiden Ländern gibt, deutet auf zusätzliche ätiologisch wirksame Fakto- 
ren hin (Wittekind et al. 2001). Der steigende Nikotinkonsum bei Frauen geht mit einer Zunahme der Inzidenz für Plattenepithelkarzinome im Kopf-Halsbereich bei Frauen einher. Früher betrug das Verhältnis zwischen Männern und Frauen etwa 7:1, während aktuell bereits jeder 3. bis 4. Patient mit einem Kopf-Hals-Malignom weiblich ist (Thurnher und Heiduschka 2011).

Neben den exogenen Noxen, wie Nikotin und Alkohol, beeinflussen auch individuelle Faktoren das Risiko für eine Tumorentstehung. In diesem Kontext spielen neben genetischen, immunologischen, hormonellen Faktoren auch alters- und geschlechtsspezifische Faktoren eine Rolle. Der schrittweise, durch genetische Veränderungen voran-getriebene Prozess bei der Tumorentstehung wird auch als vielstufige Karzinogenese bezeichnet (Reichert et al. 2002). Diese molekulare Mehrschrittpathogenese führt vom normalen Epithel über zunehmende Dysplasien, das Carcinoma in situ bis zum invasiven Karzinom und zur Metastasierung (Maschmeyer et al. 2011). Im Zuge dieser Entwicklung kommt es zu einer Akkumulation von genetischen Veränderungen. Zu den untergeordneten Risikofaktoren gehören berufsbedingte Noxen, wie der Umgang mit Farben, Lacken, Lösungsmitteln, Asbest und weiteren Stäuben (Wittekind et al. 2001).

\subsubsection{TNM-Klassifikation}

Zur Stadieneinteilung wird allgemein das TNM-System der UICC (Union Internationale Contre le Cancer) verwendet (Brierley et al. 2017). Diese verbindliche Klassifikation nach international gültigen Regeln gibt Auskunft über die Größe des Tumors, das Infiltrationsverhalten, die lokoregionalen Lymphknotenmetastasierungen und die möglichen Fernmetastasen (Brierley et al. 2017,S. 3-9, Ehrenfeld und Prein 2002). Die Abkürzung "TNM" steht für folgende Kriterien:

- Die T-Kategorie beschreibt die Ausdehnung des Primärtumors.

- Die N-Kategorie beschreibt den Befund der regionären Lymphknoten.

- Die M-Kategorie beschreibt den Befund der Fernmetastasen.

Wichtig ist hier die Differenzierung zwischen der klinischen (cTNM) und der pathologischen Klassifikation (pTNM) (Brierley et al. 2017, S. 4). 
Zusätzlich zur TNM-Einstufung werden in Befunden häufig weitere Faktoren angegeben:

1. Differenzierungsgrad (G1-G3)

2. Invasion in die abführenden Venen (V) oder Lymphgefäße (L)

3. R (Resttumor) bzw. r (Rezidiv)

4. Grad der Sicherheit $(\mathrm{C} 1-\mathrm{C} 5=$ Certainty-Index $)$

Das TNM-Schema ist tumor- und lokalisationsbezogen. Die Klassifikation für Oropharynxkarzinome ist in der folgenden Tabelle 1 aufgeführt.

Tabelle 1: TNM-Klassifikation für Oropharynxkarzinome (Brierley et al. 2017,S. 24)

\begin{tabular}{|c|c|}
\hline T-Stadium & \\
\hline $\mathrm{T} 1$ & $<2 \mathrm{~cm}$ \\
\hline $\mathrm{T} 2$ & $>2$ bis $4 \mathrm{~cm}$ \\
\hline $\mathrm{T} 3$ & $>4 \mathrm{~cm}$ \\
\hline T4a & $\begin{array}{l}\text { Knocheninfiltration, Beteiligung der intrinsischen } \\
\text { Zungenmuskulatur, Kieferhöhle, Gesichtshaut }\end{array}$ \\
\hline $\mathrm{T} 4 \mathrm{~b}$ & Kaumuskulatur, Pterygoid, Schädelbasis, A. carotis interna \\
\hline N-Stadium & \\
\hline N1 & ipsilateraler $>3 \mathrm{~cm}$ \\
\hline $\mathrm{N} 2$ & $\begin{array}{l}\text { a ipsilateraler LK zwischen } 3 \text { und } 6 \mathrm{~cm} \\
\text { b ipsilateraler unter } 6 \mathrm{~cm} \\
\text { c bilateraler, kontralateral unter } 6 \mathrm{~cm}\end{array}$ \\
\hline $\begin{array}{l}\text { N3a } \\
\text { N3b }\end{array}$ & $\begin{array}{l}>6 \mathrm{~cm} \text { ohne extranodale Ausbreitung } \\
\text { in einem oder mehreren Lymphknoten mit klinischer } \\
\text { extranodaler Ausbreitung }\end{array}$ \\
\hline M-Stadium & \\
\hline M0 & keine Fernmetastasen \\
\hline M1 & Fernmetastasen \\
\hline
\end{tabular}


Anhand der TNM-Klassifikation kann eine Stadieneinteilung vorgenommen werden, die Aussagen zur Prognose sowie zum Erfolg einer eventuellen Therapie zulässt (Tabelle 2).

Tabelle 2: Stadieneinteilung nach der TNM-Klassifikation (Brierley et al. 2017,S. 28)

\begin{tabular}{|l|l|l|l|}
\hline Stadium & T & N & M \\
\hline 0 & Tis & N0 & M0 \\
\hline I & T1 & N0 & M0 \\
\hline II & T2 & N0 & M0 \\
\hline III & T1, T2 & N1 & M0 \\
& T3 & N0, N1 & M0 \\
\hline IVa & T1, T2, T3 & N2 & M0 \\
& T4a & N0, N1, N2 & M0 \\
\hline IVb & Jedes T & N3 & M0 \\
& T4b & Jedes N & M0 \\
\hline IVc & Jedes T & Jedes N & M1 \\
\hline
\end{tabular}

\subsubsection{Standardtherapie}

Die fundamentale Therapiesäule bei Plattenepithelkarzinomen der Mundschleimhaut ist das chirurgische Vorgehen (Frerich 2010). Die S3 Leitlinie betont den Primat der chirurgischen Resektion und formuliert Empfehlungen zur Primärtumorentfernung und zur Lymphknotendissektion in Abhängigkeit vom Tumorstadium (S3-Leitlinie Mundhöhlenkarzinom 2012). Eine adjuvante Radiotherapie oder kombinierte Radiochemotherapie kommt in Abhängigkeit vom Stadium zum Einsatz. Neben der Stadieneinteilung ist die Beurteilung der individuellen Situation, wie des Alters und des Allgemeinzustands des Patienten, ausschlaggebend für die Wahl der Therapie. Bei der Entscheidung über die chirurgische Therapie sollte die postoperative Lebensqualität berücksichtigt werden. Aufgrund fehlender Evidenzen für die Überlegenheit einer bestimmten Therapieform werden lediglich Empfehlungen zur Therapiewahl ausgesprochen. Ziel der chirurgischen Therapie ist die Schaffung tumorfreier Resektionsränder (R0). Bei bösartigen Tumoren erfolgt die radikale Tumorentfernung mit einem auf die Tumorentität abgestimmten Sicherheitsabstand. Bei Mundhöhlenkarzinomen werden Sicherheitsabstände von 1-2 cm gefordert (Ehrenfeld und Prein 2002). Primärtumor der Größen T1 und T2 können in 80-90\% mit einer lokalen Exzision erfolgreich behandelt werden. Dagegen sollte bei fortgeschrittenen Karzinomen (T3, T4) ein multimodales Behandlungskonzept aus einer chirurgischen Therapie und einer Radiotherapie oder Radiochemotherapie gewählt werden (Lefebvre et al. 1994). 
Die kombinierte Radiochemotherapie führte im Vergleich zur Radiotherapie bei T3 und T4 Tumoren zu einer Verbesserung der Fünf-Jahres-Überlebensrate (Garden 2004). Die S3Leitlinie zur Diagnostik und Therapie von Mundhöhlenkarzinomen (2012) empfiehlt eine postoperative Radio- oder Radiochemo-therapie bei fortgeschrittenen Plattenepithelkarzinomen (T3 und T4), bei knappen oder positiven Resektionsrändern sowie bei einem Lymphknotenbefall. Integraler Bestandteil des Therapiekonzeptes bei Mundhöhlenkarzinomen ist die Lymphknotendissektion (S3-Leitlinie Mundhöhlenkarzinom 2012). Dabei wird zwischen der modifizierten radikalen und der selektiven Neck-Dissection unterschieden.

Laut S3-Leitlinie empfiehlt sich aus prophylaktischen Gründen bei Patienten mit cN0Halsbefunden (cNO beschreibt den klinisch unauffälligen Halsbefund; kein Verdacht auf regionär befallene Lymphknoten) eine elektive Neck-Dissection, da das Risiko von okkulten Metastasen besteht. Im Falle des Mundhöhlenkarzinoms ist eine Ausräumung der Level I-III zu empfehlen; nur selten ist das Level IV betroffen. Bei klinisch und radio-logisch auffälligem Halsbefund wird in der Regel eine modifizierte radikale Neck-Dissection (Abk.: MRND) im Sinne der Ausräumung von Level I-V vorgenommen (Frerich 2010).

Nach chirurgischer Entfernung des Primärtumors ist in den meisten Fällen eine rekonstruktive Maßnahme mit dem Ziel des Erhalts, der Wiederherstellung der Kau-, Sprech- und Schluckfunktion sowie der Gesichtsästhetik erforderlich. Zur Rekonstruktion kommen lokale Lappenplastiken, freie Haut-, Schleimhaut- oder Knochentransplantationen oder mikrovaskuläre Gewebetransfers infrage.

Ein wesentlicher Bestandteil der gesamten Therapie ist eine regelmäßige Tumornachsorge. Diese sollte im ersten Jahr postoperativ monatlich erfolgen, im zweiten Jahr alle zwei Monate und im dritten Jahr alle drei Monate. Danach ist eine halbjährige Untersuchung ausreichend. Die Lebensqualität der Patienten sollte in regelmäßigen Abständen erfragt werden. Nach dem fünften Jahr sollten die üblichen Früherkennungsmaßnahmen zur Anwendung kommen. Die Tumornachsorge ist deshalb von extremer Wichtigkeit, da es bei circa einem Fünftel der Patienten mit einem Mundhöhlenkarzinom zu einem lokalen Tumorrezidiv kommt. 


\section{ZIELSETZUNG}

Trotz der intensiven Forschungsbemühungen der letzten Jahre ist die PTR nicht vollständig ergründet.

Die retrospektive Analyse soll anhand eines Patientenkollektivs mit Plattenepithelkarzinomen im Kopf-Hals-Bereich durchgeführt werden. Dabei sollen die PTR und die Lebensqualität anhand etablierter Fragebögen bis zu einem Jahr nach dem Trauma bestimmt werden. Folgende Fragestellungen stehen im Fokus der Analyse:

- Verändern sich die wahrgenommene PTR und die Lebensqualität in dem Untersuchungszeitraum?

- Beeinflussen Patientencharakteristika, wie Alter, Geschlecht, Familienstand und Religiosität, die PTR und/oder die Lebensqualität?

- Besteht ein Zusammenhang zwischen der PTR und der Lebensqualität?

- Üben die Tumorklasse (pTNM-Klassifikation) und/oder die angewandten Therapien einen Einfluss auf die Lebensqualität aus? 


\section{PATIENTENGUT UND METHODIK}

\subsection{Patientengut}

Zur retrospektiven Auswertung wurden alle Patienten mit einem primären Plattenepithelkarzinom im Kopf-Hals-Bereich, die sich im Zeitraum zwischen Januar 2011 und Juni 2012 in der Mund-Kiefer-Gesichtschirurgie des Universitätsklinikum Göttingen in Therapie befanden, herangezogen.

Seit 2007 sind in dieser Abteilung die routinemäßige Evaluation der Lebensqualität und seit 2010 die Evaluation der PTR Bestandteile der Aufnahmeuntersuchung und der Verlaufsbeobachtung.

Die retrospektive Untersuchung wurde von der Ethikkommission der Universität Göttingen unter der Nummer 27/4/06 positiv bewertet.

\subsection{Studiencharakteristika}

Bei der Studie handelt es sich um eine retrospektive Analyse mit anonymisierten Daten. Die Erfassung der Lebensqualität und PTR erfolgte mithilfe standardisierter Fragebögen, die den Patienten routinemäßig während des Klinikaufenthalts und der Nachsorgeuntersuchungen ausgehändigt wurden. Die Teilnahme an dieser Standardbefragung im Rahmen der Tumortherapie war freiwillig. Die Patienten wurden über den Zweck der Befragung informiert.

Zum Ausschluss eines Patienten führte:

unvollständige Fragebögen

$>$ unregelmäßige Teilnahme

\subsection{Datenerhebung}

Einige Patienten waren physisch nicht dazu in der Lage, an der Befragung teilzunehmen, oder sie lehnten eine Einwilligung nach erfolgter Aufklärung ab. Ein Teil der Patienten willigte zunächst ein, an der Befragung teilzunehmen, füllte die benötigten Fragebögen allerdings nicht regelmäßig oder nur unvollständig aus. 
Aus den Akten der Patienten wurden folgende Daten generiert:

1. Alter (sieben Patienten $\leq 60$ Jahre, acht $>60$ Jahre)

2. Geschlecht (sechs Frauen, neun Männer)

3. Familienstand (sieben Patienten verheiratet, 8 nicht verheiratet)

4. Konfessionszugehörigkeit (fünf Patienten konfessionslos, 10 konfessionszugehörig)

5. Lokalisation des Primärtumors

6. pTNM-Klassifikation von 2010

7. Rekonstruktion

8. Adjuvante Therapie

Die Patienten wurden bei ihrer stationären Aufnahme über die routinemäßige Erfassung ihrer Daten sowie über deren anonymisierte Auswertung aufgeklärt. Teilnehmende Patienten füllten präoperativ einen Lebensqualitäts-Fragebogen aus.

Bei den monatlichen Nachsorgeuntersuchungen wurde die Lebensqualität der Patienten ebenfalls per Fragebogen erhoben. Zusätzlich füllten die Patienten postoperativ jeweils Fragebögen nach einem Monat, sechs Monaten und zwölf Monaten zur PTR aus. Insgesamt mussten also 15 Fragebögen vollständig und entsprechend den Zeitpunkten ausgefüllt werden. Die genaue Erläuterung der Fragebögen und des Vorgangs, wie diese ausgewertet wurden, ist aus dem folgenden Kapitel 3.4 zu entnehmen.

Aufgrund der genannten Ausschlusskriterien und der geringen Bereitschaft der Patienten zur Teilnahme an der Befragung und der hohen Anzahl an Fragebögen $(=15)$ reduzierte sich das Patientenkollektiv.

Von den 30 Tumorpatienten, die sich zunächst zum Ausfüllen der Bögen bereit erklärt hatten, füllten nur 15 Patienten alle Fragebögen vollständig aus. Insgesamt wurden während des Beobachtungszeitraums mehr als 60 Patienten behandelt. 


\subsection{Fragebögen}

\subsubsection{Fragebogen zur Lebensqualität}

Die Befragung der Patienten erfolgte mittels des UW-QOL v4-Fragebogens (S. 26-30 Abbildung 3). Hierbei handelt es sich um einen seit 1993 erhältlichen Fragebogen zur gesundheitsbezogenen Lebensqualität der Universität Washington (Rogers et al. 2010). Ein Vorteil dieses Fragebogens besteht darin, dass er in die beiden Subskalen: a) physische Funktion und b) sozial-emotionale Funktion unterteilt ist. Die Identifikation von Subskalen kann unter Umständen zu einer verbesserten Änderungssensibilität und einem höheren Genauigkeitsgrad führen (Rogers et al. 2010). Die aktuelle Version 4 des UW-QOL v4Fragebogens besteht aus zwölf Fragen mit jeweils 3-6 Antwortmöglichkeiten.

Die Fragen betreffen die Domänen Schmerz, Aussehen, Aktivität, Erholung, Schlucken, Kauen, Sprache, Schulter, Geschmack, Salivation, Angst und Stimmung. Zur besseren Validierung lässt sich eine Aufteilung in physische Funktion (Aussehen, Schlucken, Kauen, Sprache, Geschmack, Speichel) und sozial-emotionale Funktion (Schmerz, Aktivität, Erholung, Schulter, Stimmung, Angst) vornehmen. Diese Aufteilung wird von den Autoren deshalb als sinnhaft erachtet und ordnet z. B. die Schulter der sozial-emotionalen Funktion $\mathrm{zu}$, weil diese von den Patienten her vor allem Wichtigkeit hinsichtlich der Arbeit und Hobbies hat (Rogers et al. 2010).

Darüber hinaus sollen die Patienten bis zu drei Bereiche anzukreuzen, die für sie in der letzten Woche am bedeutendsten waren. Zusätzlich enthält der UW-QOL v4-Fragebogen drei weitere Fragen zur Lebensqualität. Die erste Frage soll herausfinden, ob und in welchem Maße sich die Lebensqualität im Verhältnis zum Zeitpunkt vor der Krebserkrankung verändert hat. Mit den anderen beiden Fragen werden die gesundheitsbezogene Lebensqualität und die Lebensqualität insgesamt herauskristallisiert. Eine Spalte für Freitext eröffnet dem Patienten die Möglichkeit, zusätzliche Punkte oder Anmerkungen hinzuzufügen. Der gesamte Fragebogen bezieht sich auf die momentane Gesundheit des Patienten und auf die Lebensqualität innerhalb der letzten sieben Tage.

Die Auswertung der Fragen 1-12 erfolgt über eine Skalierung von 0-100, um die Präsentation der Ergebnisse zu vereinfachen. Als Beispiel Frage 1: 0 bedeutet keine Schmerzen, 25 geringe Schmerzen, 50 mäßige Schmerzen, 75 starke Schmerzen (keine Schmerzmittel notwendig), 100 starke Schmerzen (nicht kontrollierbar mit Schmerzmitteln). 


\section{Patient}

Datum:

Datum der Operation:

\section{Fragebogen Lebensqualität}

Im folgenden Fragebogen werden Ihnen Fragen zu Ihrer Gesundheit und zur Lebensqualität gestellt.

Sie sollen dabei nur die letzte Woche in Betracht ziehen.

Zu jeder Frage steht Ihnen eine Auswahl an Antwortmöglichkeiten zur Verfügung. Bitte wählen Sie jeweils nur eine Antwortmöglichkeit aus.

Abbildung 3: UW-QOL v4-Fragebogen in deutscher Version. Übersetzung erfolgte durch Schliephake und Gruber. Quelle: www.headandneckcancer.co.uk 
1 Schmerz

$\Pi$ Ich habe keine Schmerzen.

$\Pi$ Ich habe geringe Schmerzen - benotige jedoch keine Schmerzmittel.

$\sqcap$ Ich habe mäige Schmerzen - benotige schwache Schmerzmittel (z. B. Paracetamol).

П Ich habe starke Schmerzen - benotige starke Schmerzmittel (z.B. Morphium).

$\Pi$ Ich habe starke Schmerzen - nicht kontrollierbar durch Schmerzmittel.

\section{Aussehen}

$\sqcap$ Es gibt keine Ánderung bzgl. meines Aussehens.

$\Pi$ Mein Aussehen hat sich geringfugig verandert.

$\sqcap$ Mein Aussehen stort mich, ich bleibe jedoch aktiv

$\sqcap$ Ich komme mir deutlich entstellt vor und beschrănke meine Aktivitaten wegen des Aussehens

$\sqcap$ Ich kann nicht mit Menschen zusammen sein wegen meines Aussehens.

\section{Aktivitat}

$\Pi$ Ich bin so aktiv wie immer

$\sqcap$ Es gibt Momente wenn ich nicht meinen alten Elan habe, aber nicht oft.

$\Pi$ Ich bin oft mûde und habe meine Aktivitàten reduziert, wenngleich ich immer noch ausgehe

$\Pi$ Ich gehe nicht aus weil ich nicht genug Kraft habe.

$\sqcap$ Ich bin gewöhnlich im Bett oder sitze im Stuhl und verlasse nicht die Wohnung.
4 Erholung
$\Pi$ Es gibt keine Einschrankungen bzgl. Erholung zu Hause oder anderswo.
$\Pi$ Es gibt einige Dinge, die ich nicht machen kann, dennoch gehe ich aus und genieße das Leben
$\sqcap$ Oftmals wunsche ich mir, ich konnte mehr ausgehen, aber ich schaffe es nicht.
$\sqcap$ Es gibt sehr starke Einschrankungen bzgl. Freizeitaktivitaten, meistens bleibe ich zu Hause
$\sqcap$ Ich kann nichts machen, was mir Spaß machen würde.
5 Schlucken
$\Pi$ Ich kann so gut schlucken wie immer
$\sqcap$ Ich kann bestimmte feste Nahrung nicht schlucken
$\sqcap$ Ich kann nur flüsige Nahrung schlucken.
$\Pi$ Ich kann nicht schlucken weil es den "falschen Weg geht" und ich mich verschlucke.

Abbildung 3: UW-QOL v4-Fragebogen in deutscher Version. Übersetzung erfolgte durch Schliephake und Gruber. Quelle: www.headandneckcancer.co.uk 
6 Kauen

$\sqcap$ Ich kann so gut kauen wie immer.

$\sqcap$ Ich kann weiche Kost zu mir nehmen, aber keine feste Nahrung kauen.

$\sqcap$ Ich kann nicht einmal weiche Kost zu mir nehmen.

7 Sprache

$\sqcap$ Meine Sprache ist die gleiche wie immer.

$\sqcap$ Ich habe Probleme bestimmte Wörter auszusprechen, werde aber am Telefon verstanden.

$\sqcap$ Nur meine Familie und Freunde können mich verstehen.

$\sqcap$ Ich werde nicht verstanden.

8 Schulter

$\sqcap \quad$ Ich habe keine Probleme mit meiner Schulter

$\sqcap$ Meine Schulter ist steif aber es hat keinen Einfluß auf meine Aktivität und Stärke.

$\sqcap$ Schmerz und Schwäche in meiner Schulter hat mich gezwungen, Beruf und Hobbies zu ändern

$\sqcap$ Ich kann nicht arbeiten oder meine Hobbies ausüben wegen Schmerzen in meiner Schulter.

9 Geschmack

$\sqcap$ Ich kann Nahrung normal schmecken.

$\sqcap$ Ich kann Nahrung meistens normal schmecken.

$\sqcap$ Ich kann nur bestimmte Nahrung schmecken.

$\sqcap$ Ich kann keine Nahrung schmecken.

10 Speichel

$\sqcap$ Mein Speichel hat eine normale Beschaffenheit

$\sqcap$ Ich habe weniger Speichel als normal, aber es ist ausreichend

$\sqcap$ Ich habe zu wenig Speichel.

$\sqcap \quad$ Ich habe keinen Speichel.

11 Stimmung

$\sqcap$ Meine Laune ist exzellent und unbeeinflußt durch meinen Tumor.

$\sqcap$ Meine Laune ist allgemein gut and nur gelegentlich beeinflußt durch meinen Tumor.

$\sqcap$ Ich bin weder in guter Laune noch depressiv wegen des Tumors.

$\sqcap$ Ich bin etwas depressiv wegen meines Tumors.

$\sqcap$ Ich bin sehr depressiv wegen meines Tumors.

Abbildung 3: UW-QOL v4-Fragebogen in deutscher Version. Übersetzung erfolgte durch Schliephake und Gruber. Quelle: www.headandneckcancer.co.uk 
12 Angst

$\sqcap$ Ich habe keine Angst wegen meines Tumors.

$\sqcap$ Ich habe ein wenig Angst wegen meines Tumors.

$\sqcap \quad$ Ich habe große Angst wegen meines Tumors.

$\sqcap$ Ich habe sehr große Angst wegen meines Tumors

13 Beschäftigung

$\sqcap \quad$ Ich arbeite Vollzeit.

$\sqcap$ Ich arbeite Teilzeit, habe aber eine feste Arbeit.

$\sqcap \quad$ Ich arbeite nur gelegentlich.

$\sqcap$ Ich bin im Ruhestand - der Ruhestand steht in keinem Zusammenhang mit der Tumorbehandlung

$\sqcap \quad$ Ich bin im Ruhestand wegen der Tumorbehandlung

$\sqcap$ Ich bin arbeitslos.

Welche der folgenden Punkte waren während der letzten Woche für Sie am wichtigsten?

Wählen Sie bis zu 3 Punkte!

\begin{tabular}{|c|c|c|}
\hline & 1 & Schmerz \\
\hline$\square$ & 2 & Aussehen \\
\hline$\square$ & 3 & Aktivität \\
\hline$\square$ & 4 & Erholung \\
\hline$\square$ & 5 & Schlucken \\
\hline$\square$ & 6 & Kauen \\
\hline$\square$ & 7 & Sprache \\
\hline$\square$ & 8 & Schulter \\
\hline & 9 & Geschmack \\
\hline$\square$ & 10 & Speichel \\
\hline$\square$ & 11. & Stimmung \\
\hline$\square$ & 12 & Angst \\
\hline & 13 & Beschäftigung \\
\hline
\end{tabular}

Abbildung 3: UW-QOL v4-Fragebogen in deutscher Version. Übersetzung erfolgte durch Schliephake und Gruber. Quelle: www.headandneckcancer.co.uk 


\section{Generelle Fragen:}

Wie geht es Innen jetzt verglichen mit den Monaten vor der Krebserkrankung? (Eine Antwort)
$\sqcap$ Viel besser
$\sqcap$ Etwas besser
$\sqcap$ Ungefähr das gleiche
$\sqcap$ Etwas schlechter
$\sqcap$ Viel schlechter

Wie beurteilen Sie ihre gesundheitsbezogene Lebensqualität während der letzten 7 Tage?
$\sqcap$ Hervorragend
$\sqcap$ Sehr gut
$\sqcap$ Gut
$\sqcap$ Zufriedenstellen
$\sqcap$ Schlecht
$\sqcap$ Sehr schlecht

Wie beurteilen Sie ihre gesamte Lebensqualität (Gesundheit, Familie, Beruf, Freunde) während der letzten 7 Tage?
$\sqcap$ Hervorragend
$\sqcap$ Sehr gut
$\sqcap$ Gut
$\sqcap$ Zufriedenstellend
$\sqcap$ Schlecht
$\sqcap$ Sehr schlecht

Bei Bedarf beschreiben Sie bitte weitere Punkte, die sie in Ihrer Lebensqualität beeinflussen und im Fragebogen nicht erwähnt wurden:

Abbildung 3: UW-QOL v4-Fragebogen in deutscher Version. Übersetzung erfolgte durch Schliephake und Gruber. Quelle: www.headandneckcancer.co.uk 


\subsubsection{Fragebogen zur Posttraumatischen Reifung}

Die PTR wird mithilfe des Fragebogens „Persönliche Posttraumatische Reifung“ (PPR) erfasst (S. 32 Abbildung 4). Hierbei handelt es sich um die deutsche Version des Selbstbeurteilungsbogens Posttraumatic Growth Inventory von Tedeschi und Calhoun. Der von Tedeschi und Calhoun (1996) entwickelte Fragebogen wurde 2001 in der deutschsprachigen Version präsentiert (Maercker und Langner 2001). Der PPR-Fragebogen enthält 21 Fragen mit jeweils sechs Antwortmöglichkeiten, anhand derer beurteilt wird, ob und in welchem Maße sich die Sichtweise des Patienten nach der Diagnose verändert hat. Der mehrdimensionale PPR-Fragebogen lässt sich in fünf Subskalen aufteilen (Maercker und Langner 2001): „Intensivierung persönlicher Beziehungen“ (Fragen 6, 8, 9, 15, 16, 20, 21), „Neue Möglichkeiten“ (Fragen 3, 7, 11, 14, 17), „Persönliche Stärke“ (Fragen 4, 10, 12 , 19), „Wertschätzung des Lebens“ (Fragen 1, 2, 3), „Religiöse Veränderungen“ (Fragen 5, 18). Maercker und Langner (2001) stellten fest, dass der PPR-Fragebogen anderen Fragebögen aufgrund seiner Mehrdimensionalität überlegen ist. Mithilfe der 21 Fragen können mehrere Inhaltsbereiche gemessen werden.

Bei den 21 Fragen stehen den Patienten jeweils sechs Antwortmöglichkeiten (,überhaupt nicht“, „,kaum“, „,ein wenig“, „ziemlich“, „stark“, „,sehr stark“) zur Verfügung. Bei der Auswertung werden für die Antworten Punkte zwischen 1 für ,überhaupt nicht“ und 6 für „sehr stark“ vergeben. Die maximal erreichbare Punktzahl beträgt demnach 126. Aufgrund der unterschiedlichen Anzahl der Fragen, die in die Subskalen eingehen, ergeben sich die folgenden maximalen Punktzahlen:

$\begin{array}{ll}\text { „Intensivierung persönlicher Beziehungen“ (PPR1) } & 42 \text { Punkte } \\ \text { „Neue Möglichkeiten“ (PPR2) } & 30 \text { Punkte } \\ \text { „Persönliche Stärke“ (PPR3) } & 24 \text { Punkte } \\ \text { „Wertschätzung des Lebens“ (PPR4) } & 18 \text { Punkte } \\ \text { „Religiöse Veränderungen“ (PPR5) } & 12 \text { Punkte }\end{array}$

Für den Vergleich der Subskalen untereinander werden die erreichten Punktzahlen durch die Anzahl der Fragen geteilt. 


\section{Posttraumatische Veränderungen (PPR) \\ Tedeschi \& Calhoun, 1996, dt. Maercker \& Langner, 2001}

Im Folgenden geht es noch einmal darum, welche Veränderungen sich in Ihrem Leben durch das am meisten belastende Ereignis ergeben haben und wie stark Sie diese einschätzen.

\begin{tabular}{|l|l|l|l|l|l|}
\hline $\begin{array}{l}\text { Über } \\
\text { haupt } \\
\text { nicht }\end{array}$ & kaum & $\begin{array}{l}\text { ein } \\
\text { wenig }\end{array}$ & $\begin{array}{l}\text { ziem- } \\
\text { lich }\end{array}$ & stark & $\begin{array}{l}\text { sehr } \\
\text { stark }\end{array}$ \\
\hline
\end{tabular}

1.Ich habe neue Vorstellungen darüber, was im Leben wichtig und vorrangig ist.

2.Ich habe ein neues Gefühl dafür, wie wertvoll mir mein Leben ist.

3.Ich entwickelte neue Interessen.

4.Ich entwickelte ein Gefühl des Selbstvertrauens.

5.Ich habe jetzt ein größeres Verständnis für religiöse und geistige Dinge.

6.Ich weiß jetzt, daß ich in schweren Zeiten auf andere Menschen zählen kann.

7. Ich beschritt einen neuen Weg in meinem Leben.

8.Ich entwickelte einen Sinn für die Verbundenheit mit anderen.

9.Ich will meine eigenen Gefühle mehr ausdrücken.

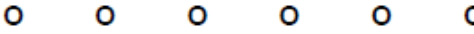

10.Ich weiß jetzt, daß ich mit Schwierigkeiten umgehen kann.

11.Ich fange mehr mit meinem Leben an.

$0 \quad 0 \quad 0 \quad 0 \quad 0 \quad 0$

12.Ich kann besser akzeptieren, daß ich auf die Entwicklung, die 0 viele Dinge nehmen, keinen Einfluß habe.

$\begin{array}{lllllll}\text { 13.Ich würdige jeden Tag. } & 0 & 0 & 0 & 0 & 0 & 0 \\ \text { 14.Möglichkeiten, die es sonst nicht gegeben hätte, sind jetzt für } & 0 & 0 & 0 & 0 & 0 & 0\end{array}$ mich verfügbar.

15.Ich habe Mitgefühl mit Anderen

16.Ich investiere jetzt mehr in meine Beziehungen.

$\begin{array}{llllll}0 & 0 & 0 & 0 & 0 & 0\end{array}$

$0 \quad 0000000$

$0 \quad 0 \quad 0 \quad 0 \quad 0 \quad 0$

17.Ich bin mehr dazu geneigt, Dinge zu verändern, die geändert werden müßten

18.Ich habe einen stärkeren religiösen oder spirituellen Glauben. $0 \quad \begin{array}{lllll}0 & 0 & 0 & 0\end{array}$

19.Ich entdeckte, daß ich stärker bin als ich dachte

20.Ich erfuhr eine Menge darüber, wie gut Menschen sind

0000000

21.Ich akzeptiere, daß ich andere brauche.

0000000

$0 \quad 0 \quad 0 \quad 0000$

Abbildung 4: PPR-Fragebogen. Tedeschi und Calhoun 1996, dt. Maercker und Langner 2001. Mit freundlicher Erlaubnis von Richard G. Tedeschi und Lawrence G. Calhoun. 


\subsection{Statistische Auswertung}

Für die Auswertung der retrospektiven Analyse wurden ausschließlich anonymisierte Daten verwendet. Zur statistischen Auswertung wurde zunächst eine Globalanalyse mit einer Repeated-measures-ANOVA und der PTR (alle Subskalen) als abhängiger Variable durchgeführt. Um herauszufinden, zu welchen Zeitpunkten der Subskalen ein signifikanter Verlauf zu erkennen ist, wurden anschließend paarweise Vergleiche der einzelnen Messzeitpunkte vorgenommen. Zur Untersuchung der möglichen Einflussfaktoren auf die PTR wurden weitere Tests durchgeführt. Bei den untersuchten Variablen handelt es sich um Geschlecht, Alter, Konfessionszugehörigkeit und Familienstand. Zur Analyse von zwei Gruppen (bspw. Geschlecht etc.) wurden t-Tests für unabhängige Stichproben verwendet (S. 42-45 Abbildung 11-13).

Darüber hinaus erfolgten eine deskriptive statistische Auswertung der prä- und der postoperativen Lebensqualität sowie die Darstellung der unter-schiedlichen Einflussgrößen auf die Lebensqualität. Die Auswertung erfolgte mit den Programmen SAS 9.4 (Cary, USA) und Statistica 10.0 (Hamburg, DE). Die Testergebnisse mit einem p-Wert $<0,05$ wurden als statistisch signifikant gewertet. 


\section{ERGEBNISSE}

Bestandteile der Studie waren zum einen die Auswertung der Fragebögen zur Persönlichen Posttraumatischen Reifung von Tedeschi und Calhoun und zum anderen die Evaluierung der UW-QOL v4-Fragebögen der Universität Washington. Zur Auswertung lagen Unterlagen von 30 Patienten mit einem primären Plattenepithelkarzinom im Kopf-Hals-Bereich, die im Zeitraum von Januar 2011 bis Juni 2012 in der Mund-Kiefer-Gesichtschirurgie des Universitätsklinikums Göttingen therapiert wurden, vor. Bei der Auswertung wurde herausgefunden, dass nicht alle Patienten die PPR- und UW-QOL v4-Fragebögen regelmäßig und vollständig ausgefüllt hatten. Aufgrund der fehlenden Compliance und der unvollständigen Fragebögen konnten lediglich 50\% der Daten $(n=15)$ ausgewertet werden.

\subsection{Beschreibung der Stichprobe}

Die Stichprobe ist nicht in allen Bereichen gleich balanciert. Es handelt sich um sechs Frauen und neun Männer. Der Altersbereich liegt zwischen 46 und 94 Jahren. Sechs der 15 Patienten sind im Alter von 60 Jahren und jünger und neun Patienten sind älter als 60 Jahre. Sieben der 15 Patienten sind verheiratet, sechs nicht verheiratet, ein Patient ist geschieden und einer verwitwet. Geschieden und verwitwet wurde in diesem Fall zu der Gruppe der Nichtverheirateten gezählt. Zehn Patienten sind konfessionszugehörig, fünf besitzen keine Konfession.

\subsection{Fragebogen zur Persönlichen Posttraumatischen Reifung}

\subsubsection{Einflussfaktor Zeit}

Zur Auswertung der Persönlichen Posttraumatischen Reifung standen pro Patient jeweils drei zu unterschiedlichen Zeitpunkten (30, 180 und 360 Tage postoperativ) ausgefüllte Fragebögen zur Verfügung.

Die Mittelwerte der PTR der 15 in die Studie eingeschlossenen Patienten in Abhängigkeit von der Zeit sind in der nachfolgenden Abbildung 5, S. 35 veranschaulicht. Dabei wurden die fünf Subskalen „Persönliche Beziehungen“, „Neue Möglichkeiten“, „Persönliche Stärke“, „Wertschätzung des Lebens“ und „Religiöse Veränderungen“ getrennt voneinander ausgewertet. Für einen Vergleich der Subskalen untereinander wurde das arithmetische 
Mittel deshalb verwendet, weil sich die Subskalen aus unterschiedlich vielen Fragen zusammensetzen.

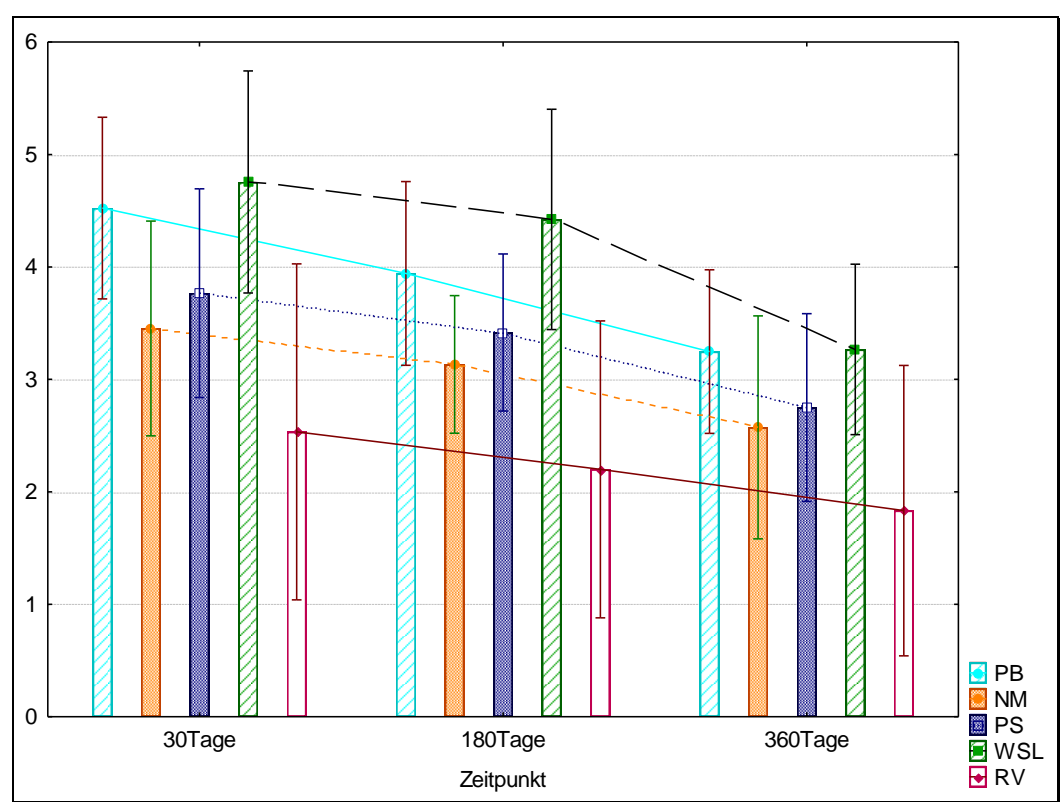

Abbildung 5: Ergebnisse des PPR-Fragebogens, getrennt nach den fünf Subskalen im zeitlichen Verlauf. Angegeben sind die Mittelwerte sowie die Standardabweichungen der 15 in die Studie eingeschlossenen Patienten zum Zeitpunkt 30, 180 und 360 Tage.

$\mathrm{PB}=$ Persönliche Beziehungen, $\mathrm{NM}=$ Neue Möglichkeiten, $\mathrm{PS}=$ Persönliche Stärke, WSL= Wertschätzung des Lebens, RV= Religiöse Veränderungen

Die Ergebnisse belegen, dass die Patienten im Durchschnitt kurz nach der Diagnose und der darauffolgenden Operation die PTR am stärksten empfanden. Die Mittelwerte aller Subskalen zeigten eine Abnahme im zeitlichen Verlauf, d. h. 30 Tage Wert > 180 Tage Wert und 180 Tage Wert > 360 Tage Wert. Dies konnte einheitlich bei allen Subskalen beobachtet werden. Nicht alle Subskalen wurden im gleichen Maße als verändert wahrgenommen. Im Bereich „Wertschätzung des Lebens“ $(M W=4,1)$ und „Persönliche Beziehungen“ $(M W=3,9)$ wurden die stärksten Veränderungen empfunden. „Persönliche Stärke“ $(M W=3,3)$ und „Neue Möglichkeiten“ $(M W=3,0)$ wurden ebenfalls als verstärkt wahrgenommen, allerdings weniger stark im Vergleich. Am niedrigsten wurden die Veränderungen im Bereich „Religiöse Veränderungen“ $(M W=2,2)$ bewertet. Der Kurvenverlauf für „Wertschätzung des Lebens“ zeigt über die drei Zeitpunkte insgesamt den stärksten Abfall. Zunächst ist eine mäßige Abnahme von 30 auf 180 Tage zu verzeichnen, wohingegen ein starker Abfall von 180 auf 360 Tage zu erkennen ist. Ähnliche Kurvenverläufe mit niedrigerem Ausgangswert zeigen „Neue Möglichkeiten“ und „Persönliche Stärke“. Der Kurvenverlauf für „Persönliche Beziehungen“ zeigt eine starke Abnahme von 30 
auf 360 Tage, welche aber über diesen Zeitraum gleichmäßiger ist. Den flachsten Kurvenverlauf zeigt die Subskala „Religiöse Veränderungen“ mit dem niedrigsten Mittelwert.

In den Abbildungen 6-10, S. 37-41 ist jede Subskala noch einmal in ihrem zeitlichen Verlauf dargestellt. Auch hier sind die Befragungszeitpunkte 30, 180 und 360 Tage. Zu jeder Subskala sind die Ergebnisse der paarweisen Vergleiche sowie der Globalanalyse aufgelistet. Die Globalanalyse ergab einen signifikanten Verlauf für 1. Persönliche Beziehungen, 2. Neue Möglichkeiten, 3. Persönliche Stärke, 4. Wertschätzung des Lebens, 5. Religiöse Veränderung $(\mathrm{p}<0,05)$. Die Ergebnisse der paarweisen Untersuchungen der Zeitpunkte 30/180 Tage, 180/360 Tage und 30/360 Tage sind bei den zugehörigen Subskalen zu finden. 


\subsubsection{Persönliche Beziehungen}

Der Verlauf in Abbildung 6 verdeutlicht die kontinuierliche Abnahme der PPR-Subskala „Persönliche Beziehungen“ im postoperativen Verlauf in einzelner Darstellung. Bei allen Patienten war der 360 Tage-Wert < 30 Tage-Wert. Untersucht wurde zunächst, ob eine globale Veränderung zwischen allen drei Zeitpunkten vorliegt (ANOVA mit Messwiederholungen). Bei einem signifikanten p-Wert $(<0,05)$ wurden anschließend paarweise Vergleiche der einzelnen Zeitpunkte durchgeführt. Für die Subskala „Persönliche Beziehungen“" resultierte ein globaler p-Wert <0,001. Die Unterschiede zwischen den restlichen Zeitpunkten ergaben ebenfalls ein signifikantes Ergebnis: 30/180 Tage ( $p=0,033), 30 / 360$ Tage $(\mathrm{p}<0,001), 180 / 360$ Tage $(\mathrm{p}<0,001)$.

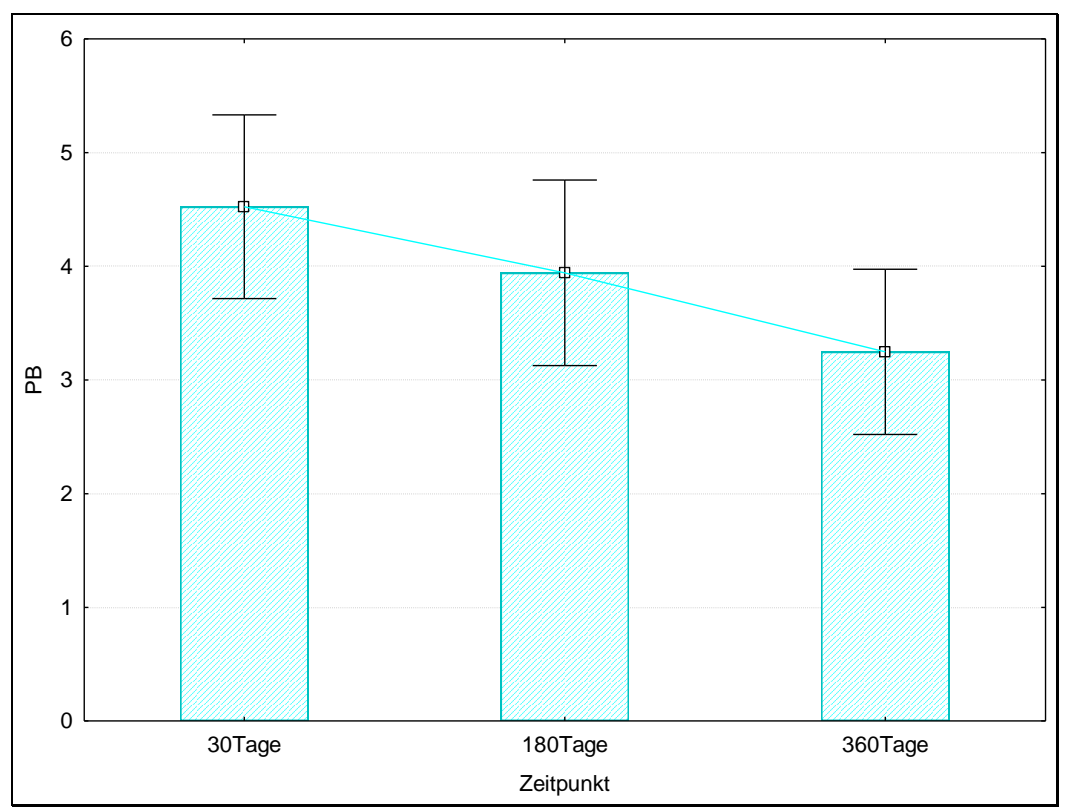

Abbildung 6: PPR-Subskala „Persönliche Beziehungen“ (PPR1) im zeitlichen Verlauf. Dargestellt sind die individuellen Messergebnisse (Mittelwert und Standardabweichung) von $n=15$ Patienten innerhalb dieser Subskala zum Zeitpunkt 30, 180 und 360 Tage.

30Tage: $M W=4,5, S D=1,6 / 180$ Tage: $M W=$ 3.9, $S D=1,6 / 360$ Tage: $M W=3,2, S D=1,4$ 


\subsubsection{Neue Möglichkeiten}

Abbildung 7 veranschaulicht die Abhängigkeit der „Neuen Möglichkeiten“ von der Zeit. Auch in dieser Subskala ist bei den Patienten ein kontinuierlicher Abfall der Beurteilung im postoperativen Verlauf zu verzeichnen. Alle Patienten bewerteten ihre neuen Möglichkeiten 360 Tage nach der Operation am schlechtesten. Die Globalanalyse ergab einen pWert $<0,001$. Die paarweisen Vergleiche wiesen allerdings nach, dass lediglich zwei der paarweisen Vergleiche signifikant sind. Diese sind die Vergleiche der Zeitpunkte von 30/360 Tage $(p<0,001)$ und 180/360 Tage $(p=0,077)$. Folgender Vergleich ergab kein signifikantes Ergebnis: 30/180 Tage $(\mathrm{p}=0,125)$.

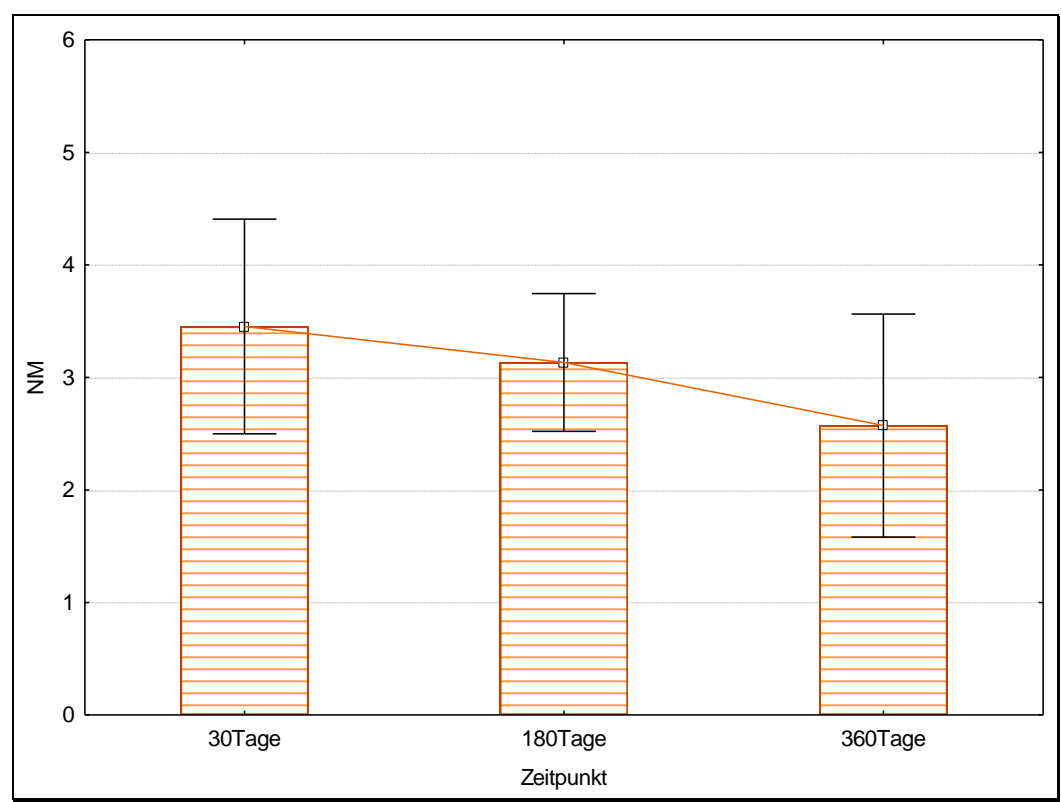

Abbildung 7: PPR-Subskala „Neue Möglichkeiten“ (PPR2) im zeitlichen Verlauf. Dargestellt sind die individuellen Messergebnisse (Mittelwert und Standardabweichung) von $\mathrm{n}=15$ Patienten zum Zeitpunkt 30, 180 und 360 Tage. 30 Tage: $M W=3,4, S D=$ 1,5 / 180 Tage: $M W=3.1, S D=1,2 / 360$ Tage: $M W=2,5, S D=2$ 


\subsubsection{Persönliche Stärke}

Abbildung 8 dokumentiert die Beurteilung der „Persönlichen Stärke“ in Abhängigkeit von der Zeit. Aus der Globalanalyse resultierte ein ebenfalls signifikantes Ergebnis mit einem p-Wert $<0,001$. Zwei der paarweisen Vergleiche ergaben ebenfalls einen p Wert $<0,05$. Diese sind der 30/360 Tage Zeitpunkt $(\mathrm{p}<0,001)$ und 180/360 Tage Zeitpunkt $(\mathrm{p}<0,001)$. Der Vergleich der Zeitpunkte 30/180 Tage ist nicht signifikant ( $\mathrm{p}=0,073)$.

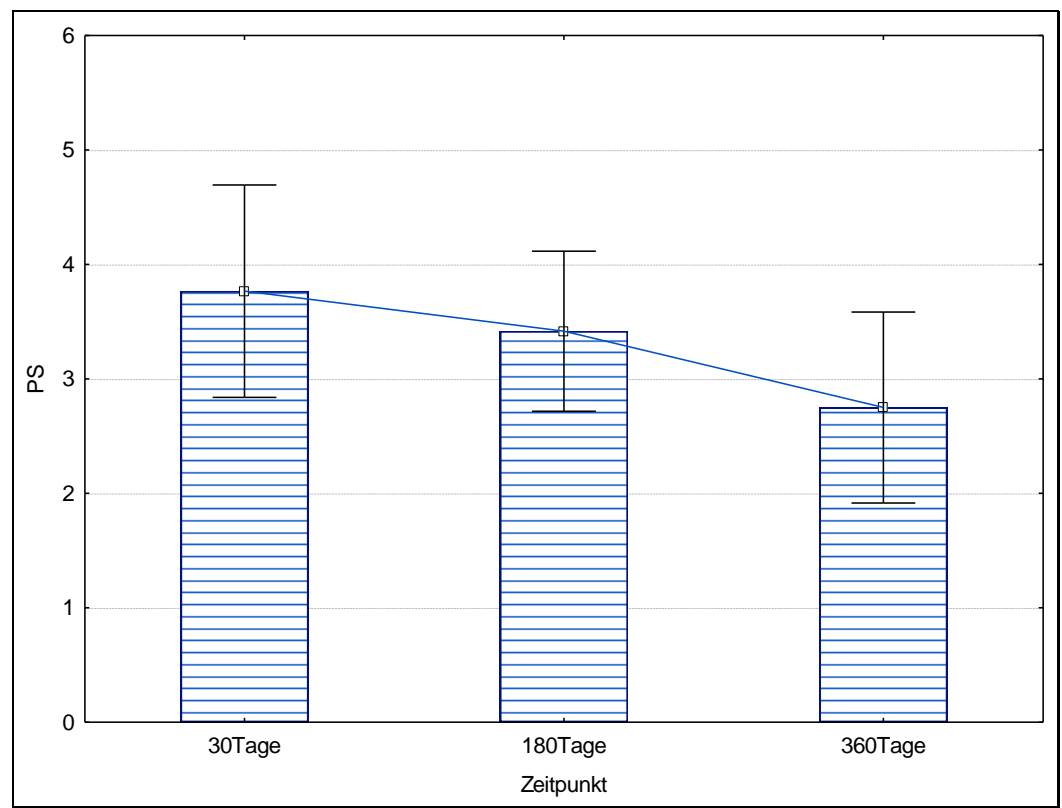

Abbildung 8: PPR-Subskala „Persönliche Stärke“ (PPR3) im zeitlichen Verlauf. Dargestellt sind die Mittelwerte und die Standardabweichungen von $n=15$ Patienten zum Zeitpunkt 30, 180 und 360 Tage.

30 Tage: $M W=3,8, S D=1,8 / 180$ Tage: $M W=3,4, S D=1,4$ / 360 Tage: $M W=2,8, S D=1,7$ 


\subsubsection{Wertschätzung des Lebens}

Abbildung 9 zeigt die Abhängigkeit der „Wertschätzung des Lebens“ im zeitlichen Verlauf. Der Kurvenverlauf verdeutlicht, wie auch in den Abbildungen 6-8, eine kontinuierliche Abnahme der erreichten Werte im zeitlichen Verlauf. Die Globalanalyse für „Wertschätzung des Lebens“ ist signifikant $(\mathrm{p}<0,001)$. Ebenso wie auch bei der Untersuchung von „Persönliche Stärke“, ergab der paarweise Vergleich der einzelnen Zeitpunkte nicht für alle drei Zeitpunkte einen $\mathrm{p}$-Wert <0,05. Der Kurvenverlauf veranschaulicht einen flacheren Verlauf vom Zeitpunkt 30/180-Tage, der p-Wert ist nicht signifikant ( $\mathrm{p}=0,202)$. Ein deutlich steilerer Abfall kann von dem 180-Tage-Wert auf den 360-Tage-Wert konstatiert werden $(p<0,001)$. Ebenfalls signifikant ist der Vergleich von 30/360 Tage $(p<0,001)$.

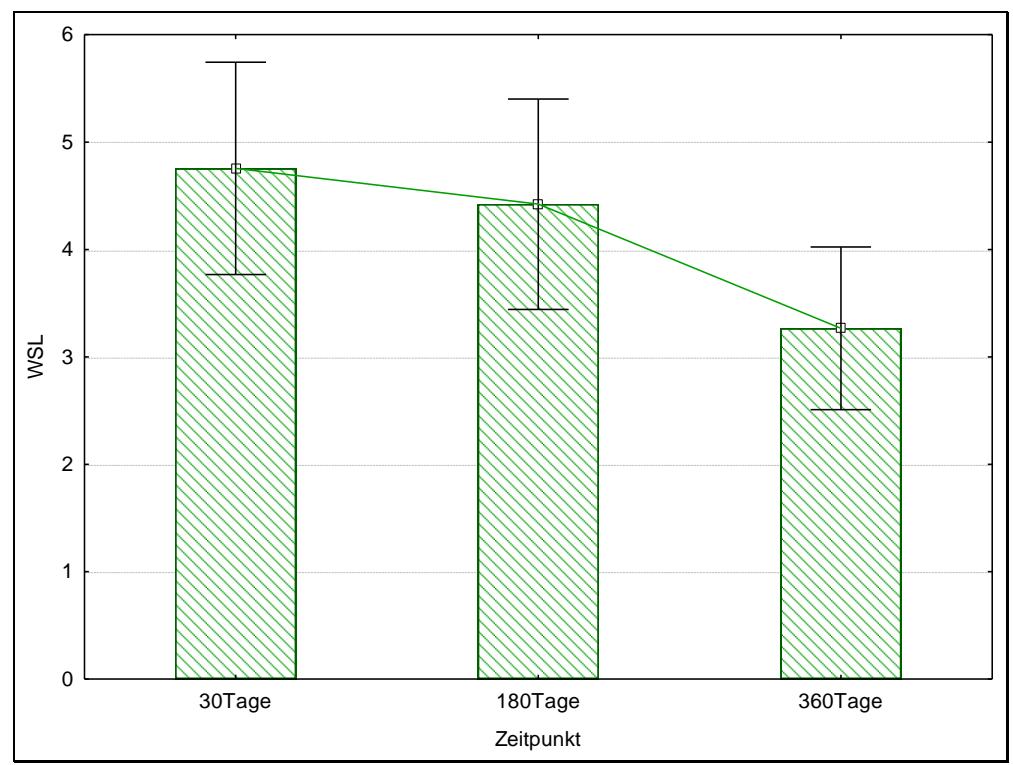

Abbildung 9: PPR-Subskala „Wertschätzung des Lebens“ (PPR4) im zeitlichen Verlauf von 30, 180 und 360 Tagen. Dargestellt sind die individuellen Messergebnisse von $\mathrm{n}=15$ Patienten. 30 Tage: $M W=4,8, S D=1,9 / 180$ Tage: $M W=4,4, S D=$ $2 / 360$ Tage: $M W=3,2, S D=1,5$ 


\subsubsection{Religiöse Veränderungen}

Abbildung 10 verdeutlicht die Bewertung der „Religiösen Veränderungen“ in Abhängigkeit von der Zeit.

Auch hier nimmt die wahrgenommene religiöse Veränderung in Abhängigkeit von der Zeit $\mathrm{ab}$, jedoch stellt sich ein wesentlich flacherer Kurvenverlauf dar. Die Globalanalyse für religiöse Veränderungen ist signifikant $(\mathrm{p}<0,05)$. Die paarweisen Vergleiche der unterschiedlichen Zeitpunkte ergab: 30/180 Tage $(p=0,136), 180 / 360$ Tage $(p=0,010), 30 / 360$ Tage ( $\mathrm{p}=0,003) .11$ der 15 Patienten empfanden eine religiöse Veränderung. Vier Patienten empfanden keinerlei religiöse Veränderungen über den gesamten Zeitraum, was dieser Darstellung allerdings nicht zu entnehmen ist.

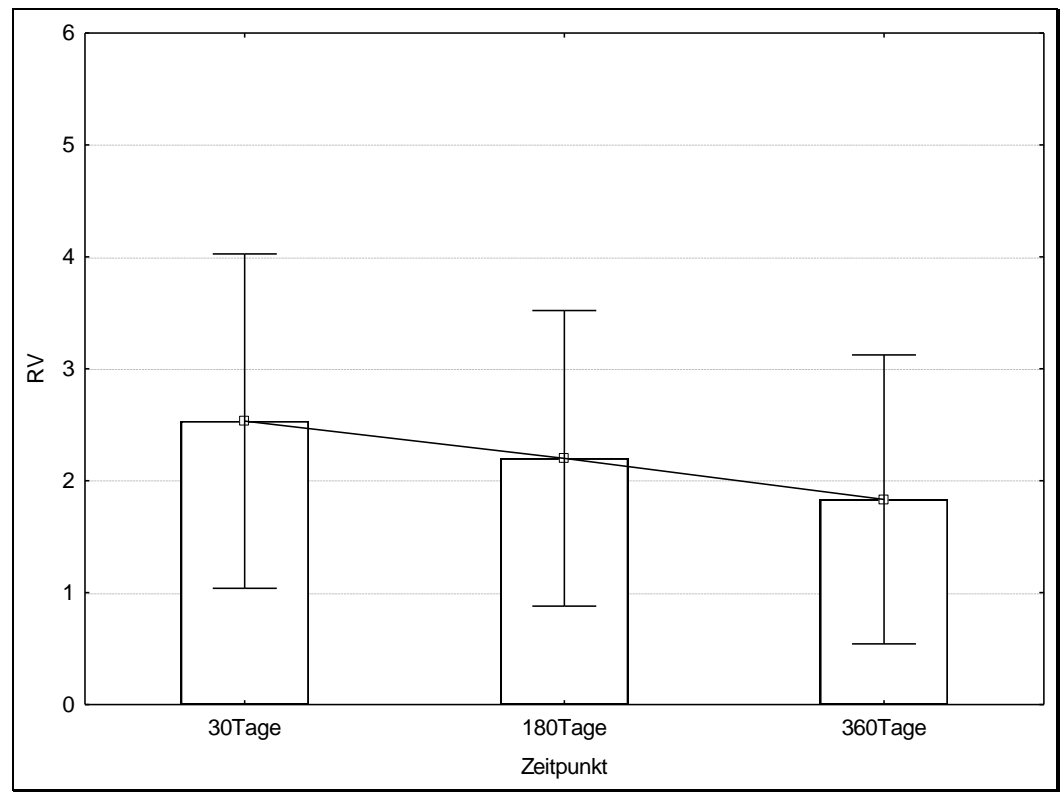

Abbildung 10: PPR-Subskala „Religiöse Veränderungen“ (PPR5) im zeitlichen Verlauf von 30, 180 und 360 Tagen. Dargestellt sind die individuellen Messergebnisse (Mittelwert und Standardabweichung) von $\mathrm{n}=15$ Patienten. 30 Tage: $M W=2,5, S D=$ 3 / 180 Tage: $M W=2,2, S D=2,6 / 360$ Tage: $M W=1,8, S D=$ 2,6 


\subsubsection{Einflussfaktor Geschlecht}

Wie bereits in Material und Methode beschrieben, wurden die möglichen Einflussfaktoren in T-Tests für unabhängige Stichproben auf einen möglichen Zusammenhang zur Posttraumatischen Reifung überprüft.

In der unten dargestellten Abbildung 11 wird der Zusammenhang zwischen PTR und Geschlecht aufgezeigt. Es konnte kein signifikanter Zusammenhang zwischen der Posttraumatischen Reifung und dem Geschlecht des Patienten eruiert werden.

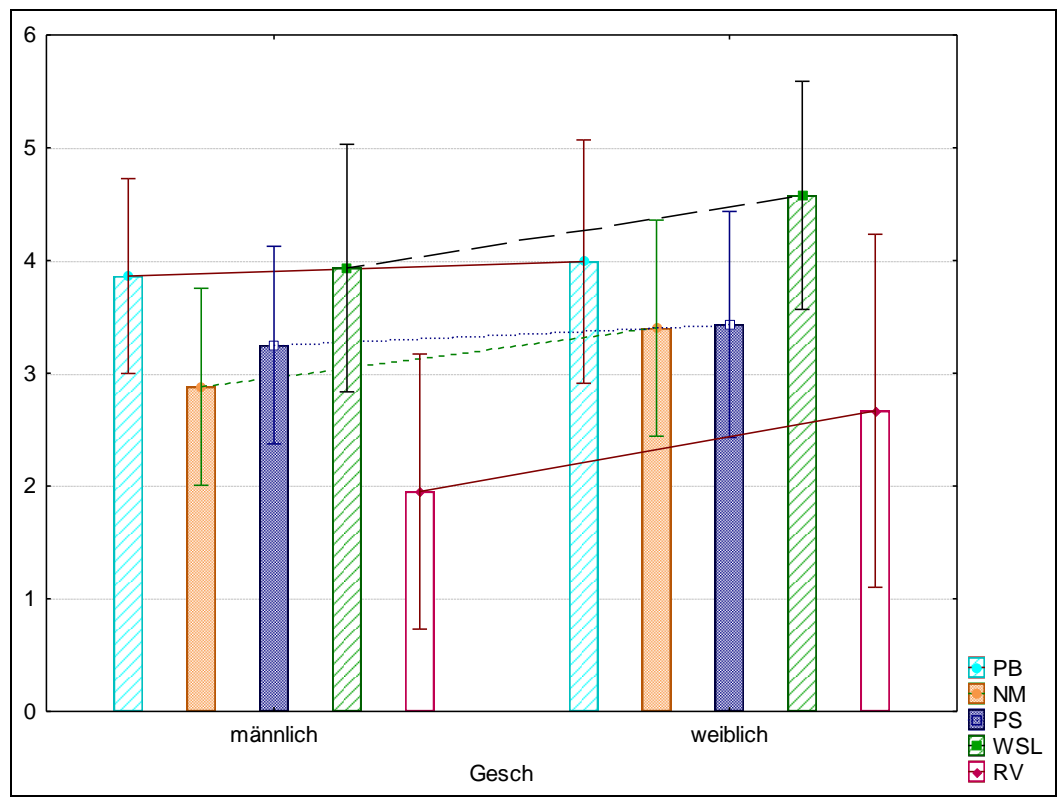

Abbildung 11: Einfluss des Geschlechts auf die posttraumatische Entwicklung. Dargestellt sind die Mittelwerte und die Standardabweichungen der fünf Subskalen des PPR-Fragebogens in Abhängigkeit vom Geschlecht des Patienten.

PB: männlich: $M W=3,9, S D=1,7$ / weiblich: $M W=4, S D=2,1$ NM: männlich: $M W=2,9, S D=1,7$ / weiblich: $M W=3,4, S D=1,9$ PS: männlich: $M W=3,2, S D=1,8$ / weiblich: $M W=3,4, S D=2$

WSL: männlich: $M W=3,9, S D=2,2$ / weiblich: $M W=4,5, S D=2$ RV: männlich: $M W=2, S D=2,3$ / weiblich: $M W=2,7, S D=3,1$ 
Wie in Abbildung 11, S. 42 zu erkennen ist, lagen die Mittelwerte bei den Frauen in den fünf Subskalen minimal höher. Den größten Unterschied zwischen den Mittelwerten der Frauen und Männer konnte bei „Religiöse Veränderung“ festgestellt werden. Trotzdem konnte durch die statistische Auswertung kein signifikanter Unterschied zwischen den männlichen und weiblichen Patienten im Hinblick auf die einzelnen Subskalen herausgefunden werden.

Der p-Wert für die Abhängigkeit von dem Geschlecht und der Wahrnehmung „Neuer Möglichkeiten“ liegt bei 0,07 und zeigt eine Tendenz dahin gehend, dass Frauen diesen Bereich stärker erleben als Männer. Die p-Werte sind der folgenden Tabelle 3 zu entnehmen.

Tabelle 3: p-Werte für den Faktor „Geschlecht“

\begin{tabular}{ll}
\hline Skala & p-Wert \\
\hline Persönliche Beziehungen & 0,667 \\
Neue Möglichkeiten & 0,075 \\
Persönliche Stärke & 0,063 \\
Wertschätzung des Lebens & 0,531 \\
Religiöse Veränderungen & 0,098 \\
\hline
\end{tabular}

\subsubsection{Einflussfaktor Alter}

Die Mittelwerte liegen in allen fünf Subskalen für Patienten im Alter unter 60 Jahre geringfügig höher. Jedoch zeigt nur die Skala „Neue Möglichkeiten“ mit einem p-Wert von 0,002, dass Patienten vor dem 60. Lebensjahr ihre „Neuen Möglichkeiten“ signifikant besser einschätzen als Patienten ab dem 60. Lebensjahr. Innerhalb der Untersuchungsgruppe $\mathrm{n}=15$ besteht somit ein signifikanter Zusammenhang zwischen Patienten $<60$ Jahre und der Wahrnehmung „Neuer Möglichkeiten“.

Bei allen anderen Subskalen lässt sich ebenfalls eine tendenzielle Abnahme der Mittelwerte in Abhängigkeit vom Alter erkennen. Patienten ab dem 60. Lebensjahr gaben im Durchschnitt geringe Werte an, was sich bei der statistischen Untersuchung allerdings als nicht statistisch signifikant herausstellte. 


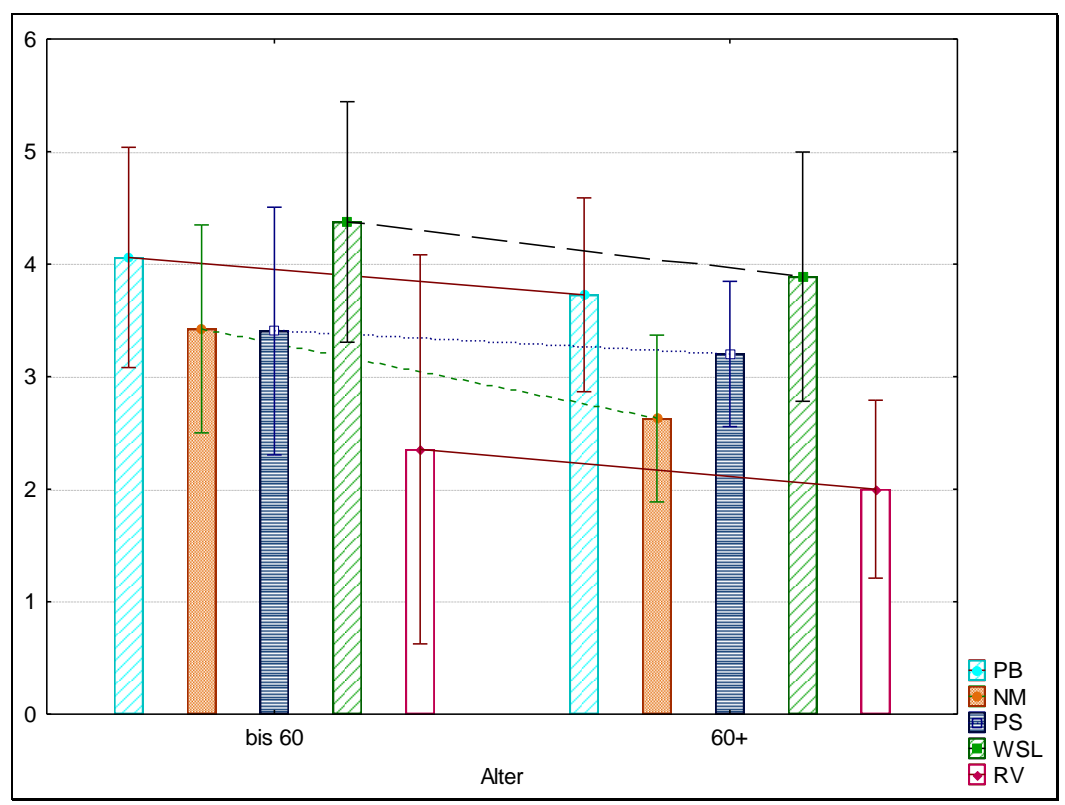

Abbildung 12: Einfluss des Alters auf die posttraumatische Entwicklung. Dargestellt sind die Mittelwerte und Standardabweichungen der Altersgruppen $<60$ Jahre und $\geq 60$ Jahre des PPRFragebogens, getrennt nach den Subskalen.

PB: bis 60: $M W=4, S D=2 / 60+: M W=3,8, S D=1,7$

NM: bis 60: $M W=3,4, S D=1,8 / 60+: M W=2,6, S D=1,4$

PS: bis 60: $M W=3,4, S D=2,2 / 60+: M W=3, S D=1$

WSL: bis 60: $M W=4,4, S D=2,1 / 60+: M W=3,9, S D=2,2$

$\mathrm{RV}$ : bis $60: M W=2,4, S D=3,4 / 60+: M W=2, S D=1,7$

Tabelle 4: p-Werte für den Faktor „Alter“

\begin{tabular}{ll}
\hline Skala & p-Wert \\
\hline Persönliche Beziehungen & 0,237 \\
Neue Möglichkeiten & $\mathbf{0 , 0 0 2}$ \\
Persönliche Stärke & 0,141 \\
Wertschätzung des Lebens & 0,461 \\
Religiöse Veränderungen & 0,393 \\
\hline
\end{tabular}

\subsubsection{Einflussfaktor Familienstand}

Der Einfluss des Familienstands auf die PTR ist nicht signifikant. Verheiratete Menschen bewerteten die Subskala „Persönliche Beziehungen“ nur geringfügig und tendenziell besser als Alleinstehende (S. 45 Abbildung 13).

Alleinstehende Personen bewerteten „Religiöse Veränderungen“ zwar etwas höher als verheiratete Menschen, aber auch hier ist der $p$-Wert nicht signifikant $(p=0,144)$. 
Die Ergebnisse für die Abhängigkeit vom Familienstand und von der posttraumatischen Reifung in fünf Subskalen sind somit insgesamt nicht signifikant. Die p-Werte können der Tabelle 5, S. 45 entnommen werden.

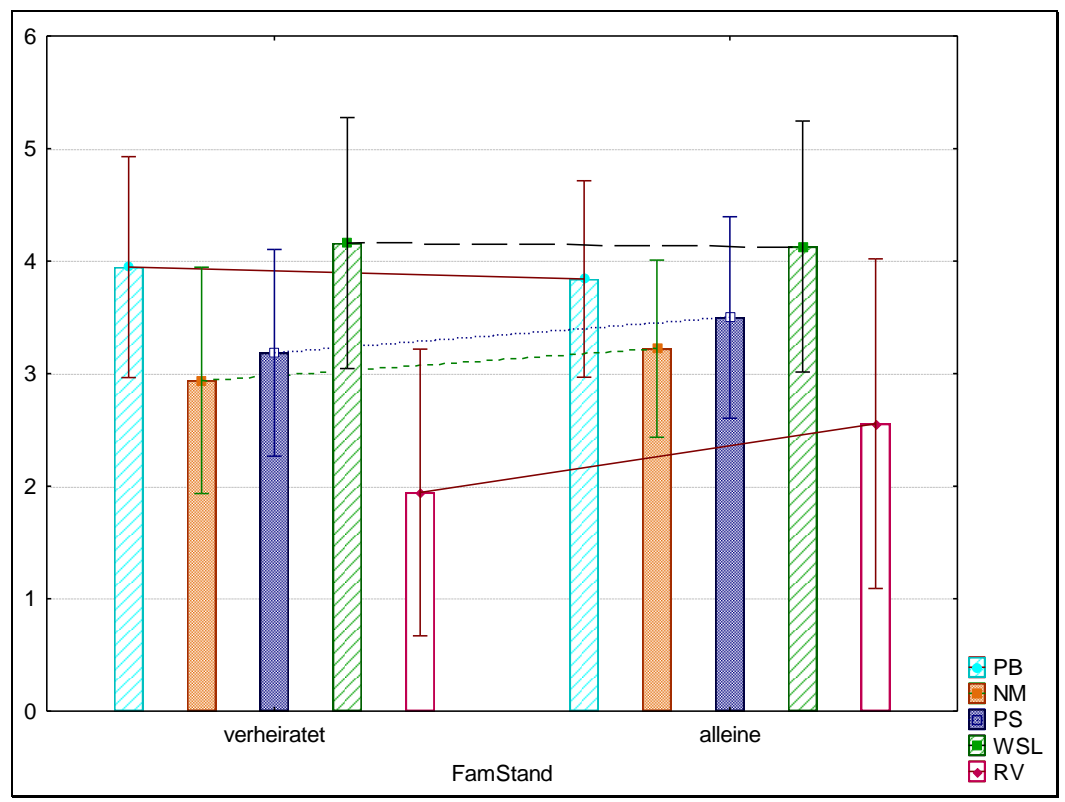

Abbildung 13: Einfluss des Familienstands auf die posttraumatische Entwicklung. Dargestellt sind die Mittelwerte und die Standardabweichungen der verheirateten und der alleinstehenden Patienten des PPR-Fragebogens, getrennt nach den fünf Subskalen.

PB: verheiratet: $M W=2,9, S D=1,9$ / alleine: $M W=3,8, S D=1,7$

NM: verheiratet: $M W=2,9, S D=2$ / alleine: $M W=3,2, S D=1,6$

PS: verheiratet: $M W=3,2, S D=1,8$ / alleine: $M W=3,3, S D=1,8$

WSL: verheiratet: $M W=4,2, S D=2,3$ / alleine: $M W=4,1, S D=2,2$

RV: verheiratet: $M W=1,9, S D=2,5 /$ alleine: $M W=2,6, S D=3$

Tabelle 5: p-Werte für den Faktor „Familienstand“

\begin{tabular}{ll}
\hline Skala & p-Wert \\
\hline Persönliche Beziehungen & 0,713 \\
Neue Möglichkeiten & 0,323 \\
Persönliche Stärke & 0,928 \\
Wertschätzung des Lebens & 0,262 \\
Religiöse Veränderungen & 0,144 \\
\hline
\end{tabular}

\subsubsection{Einflussfaktor Religion}

Diejenigen Patienten, die sich als Atheisten charakterisieren, erzielten in den ersten drei von fünf Subskalen etwas höhere Mittelwerte. Hervorzuheben ist der p-Wert aus der Skala „Persönliche Beziehungen“ mit 0,04. Dieser deutet auf einen signifikanten Zusammenhang 
zwischen der Intensivierung „Persönliche Beziehungen“ und der Religiosität hin. Nicht religiöse Patienten gaben signifikant höhere Werte in diesem Bereich an als religiöse Patienten. Religiöse Patienten gaben in der Subskala „Wertschätzung des Lebens“ und „Religiöse Veränderungen“ tendenziell höhere Werte an als nicht religiöse Patienten, was sich aber bei der statistischen Auswertung als nicht signifikant herausstellte.

Die p-Werte der anderen Subskalen sind der Tabelle 6 (s. unten) zu entnehmen und nicht signifikant.

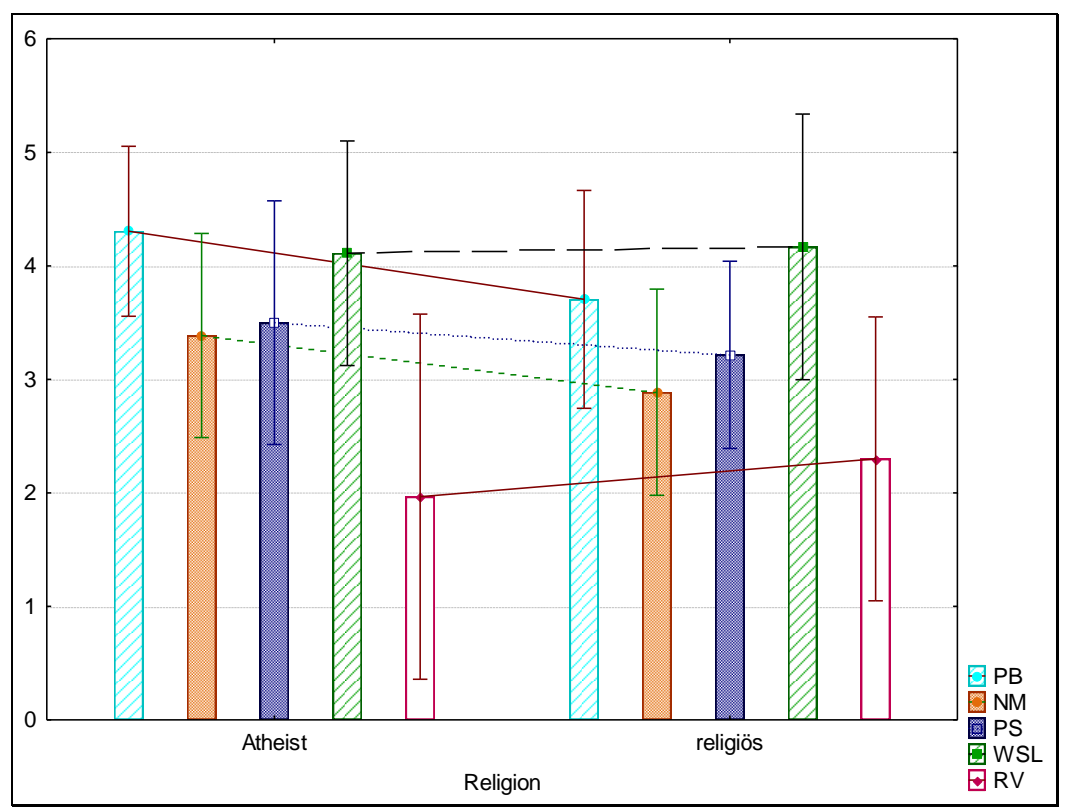

Abbildung 14: Einfluss der Religion auf die posttraumatische Entwicklung. Dargestellt sind die Mittelwerte und die Standardabweichungen von religiösen Menschen und Atheisten des PPR-Fragebogens, getrennt nach den fünf Subskalen.

PB: Atheist: $M W=4,3, S D=1,4$ / religiös: $M W=3,7, S D=2$

NM: Atheist: $M W=3,4, S D=1,8 /$ religiös: $M W=2,9, S D=$ 1,8

PS: Atheist: $M W=3,5, S D=2,1 /$ religiös: $M W=3,2, S D=1,6$

WSL: Atheist: $M W=4,1, S D=2$ / religiös: $M W=4,2, S D=2,3$

RV: Atheist: $M W=2, S D=3,3$ / religiös: $M W=2,3, S D=2,5$

Tabelle 6: p-Werte für den Faktor „Religion“

\begin{tabular}{ll}
\hline Skala & p-Wert \\
\hline Persönliche Beziehungen & $\mathbf{0 , 0 4 0}$ \\
Neue Möglichkeiten & 0,088 \\
Persönliche Stärke & 0,876 \\
Wertschätzung des Lebens & 0,332 \\
Religiöse Veränderungen & 0,448 \\
\hline
\end{tabular}




\subsection{Fragebogen zur gesundheitsbezogenen Lebensqualität (LQ)}

\subsubsection{Einflussfaktor Zeit}

Im folgenden Kapitel wird die Auswertung der gesundheitsbezogenen Lebensqualität vorgenommen. Zur Auswertung standen die UW-QOL v4-Fragebögen zur Verfügung (Kapitel 3.4.1). Neben der psychologischen Komponente, nämlich der posttraumatischen Reifung, soll die Auswertung der Lebensqualitätsfragebögen Aufschluss über die erlebte physische und sozial-emotionale Funktion vermitteln, welche sich postoperativ zum Teil in starkem Maße veränderte. Die physische und die sozial-emotionale Funktion repräsentieren gemeinsam die gesamte Lebensqualität.

Aus der Auswertung des UW-QOL v4-Fragebogens in Abhängigkeit von der Zeit resultierte, dass sich sowohl die physische als auch die sozial-emotionale Funktion (S. 48 Abbildung 15) bei allen Patienten postoperativ verschlechterten. Die meisten Patienten beurteilten ihre Lebensqualität im Unterschied zu dem Monat vor der Diagnose als etwas schlechter. Die gewählten dargestellten Zeitpunkte auf der x-Achse sind prä-op, post-op, 30, 90, 180, 270 und 360 Tage postoperativ. Der y-Achse sind die Werte der durchschnittlichen Lebensqualität zu entnehmen. Niedrige Werte bedeuten eine verbesserte, hohe Werte dagegen eine reduzierte Lebensqualität. Es konnten Werte zwischen 0-100 angegeben werden. Da keine Werte über 40 angegeben wurden, erfolgt eine dementsprechende Darstellung (S. 48 Abbildung 15).

Der Mittelwert für $\mathrm{n}=15$ weist eine minimal schlechtere sozial-emotionale Funktion (MW $=24,8, \mathrm{SD}=16,4)$ im Vergleich zur physischen Funktion nach $(\mathrm{MW}=22,5, \mathrm{SD}=16)$. 


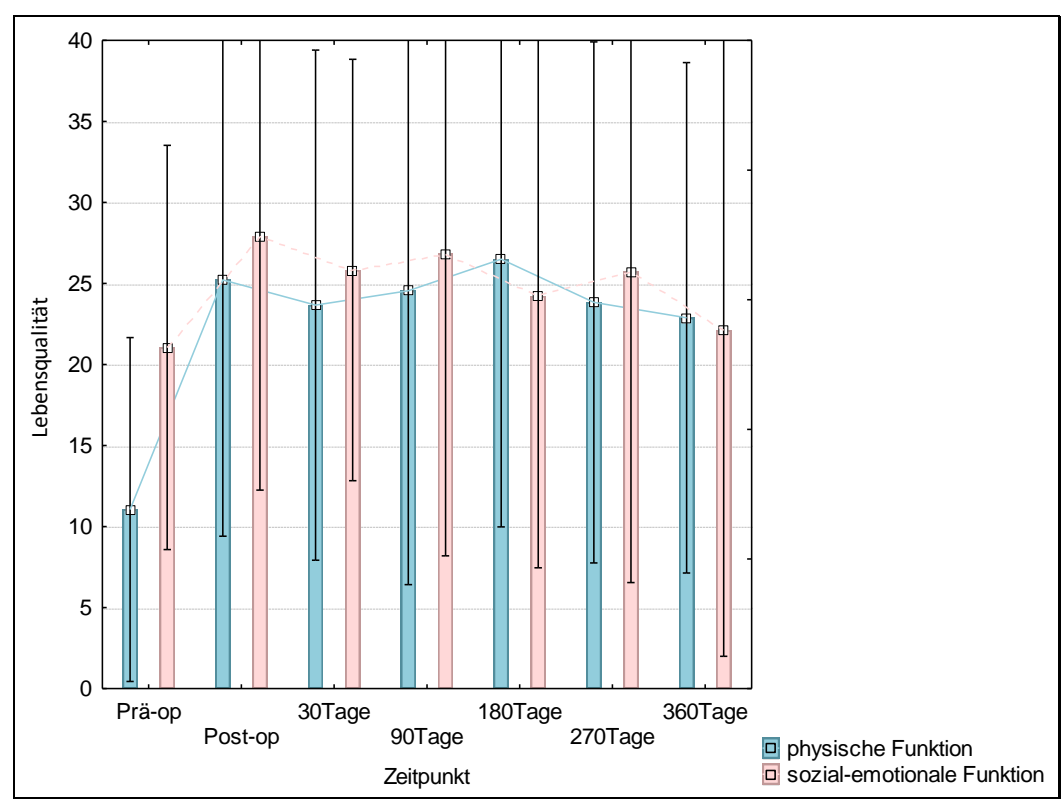

Abbildung 15: Physische und sozial-emotionale Funktion

Dargestellt sind die Mittelwerte und die Standardabweichungen von physischer und sozial-emotionaler Funktion des UW-QOL v4Fragebogens im zeitlichen Verlauf von prä-op bis 360 Tage postop. Hohe Werte in der Abbildung bedeuten eine schlechte, niedrige Werte dagegen eine gute Lebensqualität.

Physische Funktion: Prä-op: $M W=11, S D=10,6 /$ Post-op: $M W$ $=25,2, S D=15,8 / 30$ Tage: $M W=23,7, S D=15,7 / 90$ Tage: $M W=24,6, S D=18,1 / 180$ Tage: $M W=13, S D=16,5 / 270$ Tage: $M W=23,8, S D=16 / 360$ Tage: $M W=23,7, S D=15,7$ Sozial-emotionale Funktion: Prä-op: $M W=21, S D=12,5 /$ Postop: $M W=27,9, S D=15,6 / 30$ Tage: $M W=25,9, S D=13 / 90$ Tage: $M W=26,9, S D=18,6 / 180$ Tage: $M W=24,2, S D=16,8 /$ 270 Tage: $M W=25,7, S D=19,2 / 360$ Tage: $M W=25,8, S D=$ 13

Die Globalanalyse der Lebensqualität (ANOVA mit Messwiederholungen) ergab einen pWert $<0,05$ für die physische und einen p- Wert $>0,05$ für die sozial-emotionale Funktion. Ein statistisch signifikantes Ergebnis bei dem Vergleich der einzelnen Messzeitpunkte konnte somit nur bei der physischen Funktion festgestellt werden.

In den folgenden Tabellen 7 und 8 sind die Zeitpunktvergleiche mit den entsprechenden p-Werten aufgelistet. 
Tabelle 7: p-Werte der physischen Funktion

Die Zeitpunktvergleiche für die physische Funktion ergaben folgende p-Werte:

\begin{tabular}{|l|l|l|l|l|l|l|l|}
\hline & Prä-op & Post-op & $\begin{array}{l}30 \\
\text { Tage }\end{array}$ & $\begin{array}{l}90 \\
\text { Tage }\end{array}$ & $\begin{array}{l}180 \\
\text { Tage }\end{array}$ & $\begin{array}{l}270 \\
\text { Tage }\end{array}$ & $\begin{array}{l}360 \\
\text { Tage }\end{array}$ \\
\hline Prä-op & & & & & & & \\
\hline Post-op & $\mathbf{0 , 0 0 3 4}$ & & & & & & \\
\hline 30 Tage & $\mathbf{0 , 0 0 3 2}$ & 0,5696 & & & & & \\
\hline 90 Tage & $\mathbf{0 , 0 0 5 7}$ & 0,8587 & 0,7824 & & & & \\
\hline 180 Tage & $\mathbf{0 , 0 0 2 5}$ & 0,7865 & 0,3450 & 0,2910 & & & \\
\hline 270 Tage & $\mathbf{0 , 0 0 1 7}$ & 0,7207 & 0,9397 & 0,7718 & 0,1798 & & \\
\hline 360 Tage & $\mathbf{0 , 0 0 4 0}$ & 0,6121 & 0,8044 & 0,0618 & 0,1286 & 0,4712 & \\
\hline
\end{tabular}

Tabelle 8: p-Werte der sozial-emotionalen Funktion

Die Zeitpunktvergleiche für die sozial-emotionale Funktion ergaben folgende p-Werte:

\begin{tabular}{|l|l|l|l|l|l|l|l|}
\hline & Prä-op & Post-op & $\begin{array}{l}30 \\
\text { Tage }\end{array}$ & $\begin{array}{l}90 \\
\text { Tage }\end{array}$ & $\begin{array}{l}180 \\
\text { Tage }\end{array}$ & $\begin{array}{l}270 \\
\text { Tage }\end{array}$ & \begin{tabular}{l} 
Tage \\
\hline Prä-op
\end{tabular} \\
\hline Post-op & 0,1406 & & & & & & \\
\hline 30 Tage & 0,2188 & 0,3204 & & & & & \\
\hline 90 Tage & 0,2716 & 0,7391 & 0,6788 & & & & \\
\hline 180 Tage & 0,5344 & 0,2264 & 0,4582 & 0,2863 & & & \\
\hline 270 Tage & 0,4028 & 0,5860 & 0,9710 & 0,7468 & 0,5182 & & \\
\hline 360 Tage & 0,3810 & 0,2594 & 0,3180 & 0,3402 & 0,5717 & $\mathbf{0 , 0 4 8 6}$ & \\
\hline
\end{tabular}

Der Verlauf der beiden Graphen in Abbildung 15 (S. 48) zeigt einen deutlichen Anstieg des Graphen von prä- auf postoperativ, was eine Verschlechterung der Lebensqualität bedeutet.

Der Mittelwert bei der Untersuchung der physischen Funktion führte zu einem Anstieg von 11 Punkten präoperativ auf 25 Punkte postoperativ, woraus eine deutliche Verschlechterung der physischen Lebensqualität resultiert. Dieser Anstieg ist signifikant ( $p=0,003)$. Die Patienten empfanden ihre Lebensqualität nach der Operation als bedeutend schlechter. 
Bei der Untersuchung der sozial-emotionalen Funktion ergab sich ebenfalls ein Anstieg von präoperativ (21 Punkte) zu postoperativ (28 Punkte), ein Ergebnis, das allerdings nicht signifikant ist $(\mathrm{p}=0,140)$ (Globalanalyse $\mathrm{p}>0,05)$.

Beim Vergleich der Zeitpunkte präoperativ und 360 Tage postoperativ ergab die physische Funktion einen p-Wert von 0,004 und die sozial-emotionale Funktion von 0,381.

Zunächst wird eine differenzierte Auswertung der Lebensqualität in Abhängigkeit von den möglichen Einflussfaktoren vorgenommen, wie Geschlecht, Alter, Familienstand und Religion der Patienten.

In den darauffolgenden Kapiteln 4.3.6 und 4.3.7 werden die physische und die sozialemotionale Funktion jeweils einzeln in ihrem Verlauf mit den entsprechenden Unterfunktionen dargestellt und ausgewertet.

\subsubsection{Einflussfaktor Geschlecht}

Bei der Überprüfung der möglichen Abhängigkeitsfaktoren wurden die gleichen Charakteristika wie bei der Posttraumatischen Reifung ausgewählt. In den Abbildungen 16-19 (S. 50-53) sind die Ergebnisse dieser Untersuchung veranschaulicht.

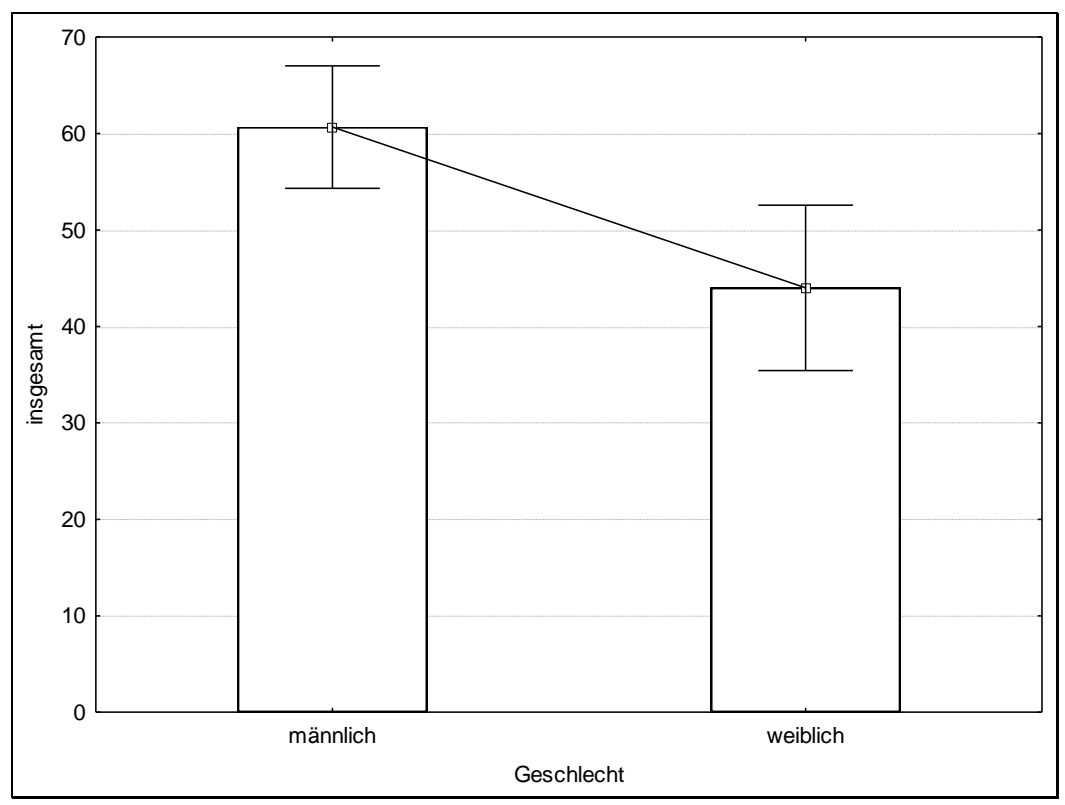

Abbildung 16: Einflussfaktor Geschlecht

Dargestellt sind die Mittelwerte und die Standardabweichungen der geschlechtergetrennten Auswertung des UW-QOL v4-Fragebogens zur Lebensqualität insgesamt über den gesamten Zeitraum. Männlich: $M=60,7, S D=6,2 /$ Weiblich: $M=44, S D=8$

Aus der statistischen Auswertung ist zu entnehmen, dass Männer im Gegensatz zu Frauen eine tendenziell schlechtere Lebensqualität angeben $(p=0,06)$. Wenn dieses Faktum auch 
nicht als signifikant zu bewerten ist, so lässt sich dennoch eine deutliche Tendenz erkennen (Abbildung 16).

\subsubsection{Einflussfaktor Alter}

Bei der Auswertung des Einflussfaktors Alter wird evident, dass die Mittelwerte bei den älteren Patienten ( $\geq 60$ Jahre) nur eine geringfügig schlechtere Lebensqualität ergaben als bei den jüngeren Patienten (< 60 Jahre).

Die statistische Auswertung wies jedoch keinen signifikanten Unterschied zwischen der Lebensqualität und den beiden Altersgruppen nach ( $\mathrm{p}=0,261)$ (Abbildung 17).

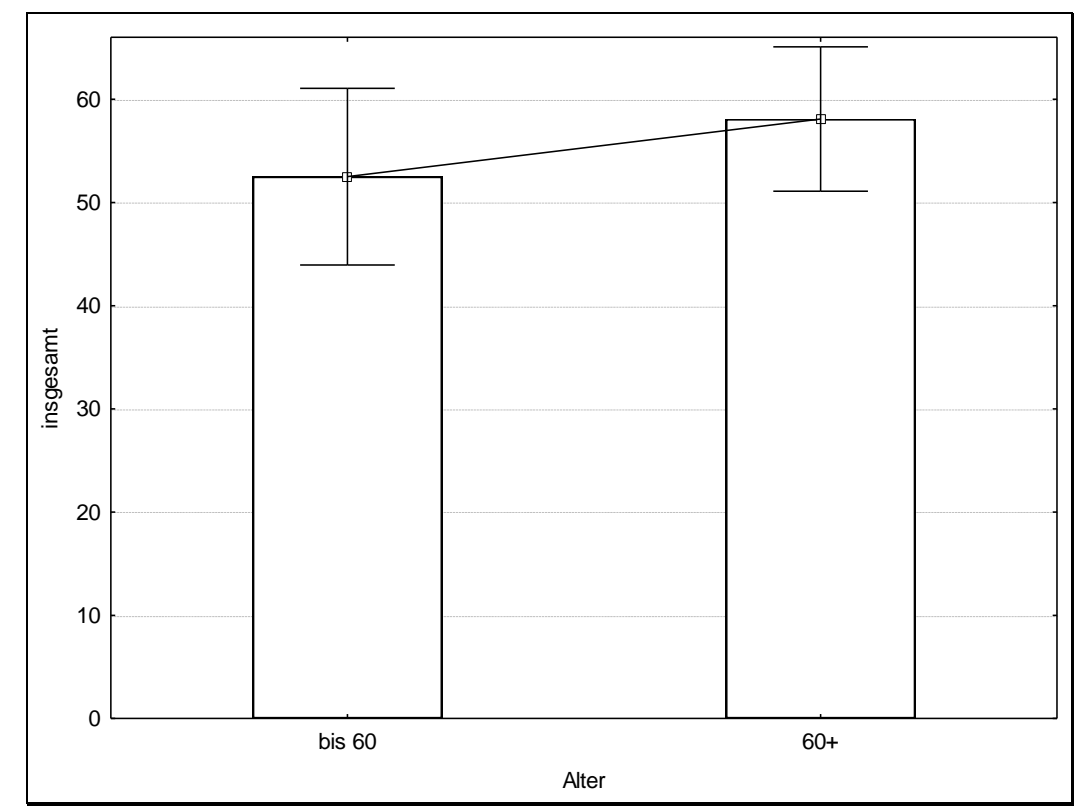

Abbildung 17: Einflussfaktor Alter

Dargestellt sind die Mittelwerte und die Standardabweichungen der Lebensqualität insgesamt (UW-QOL v4-Fragebogen) über den gesamten Zeitraum in Abhängigkeit von den Altersgruppen $<60$ Jahre und $\geq 60$ Jahre. Bis 60: $M W=52,5, S D=17,1 / 60+: M W=$ $58, S D=6,7$ 


\subsubsection{Einflussfaktor Familienstand}

Alleinstehende Patienten bewerteten ihre Lebensqualität im Durchschnitt in geringfügigem Umfang schlechter. Die Auswertung des UW-QOL v4-Fragebogens nach Familienstand ergab jedoch keine signifikanten Unterschiede zwischen verheirateten und alleinstehenden Patienten ( $\mathrm{p}=0,113)$ (Abbildung 18).

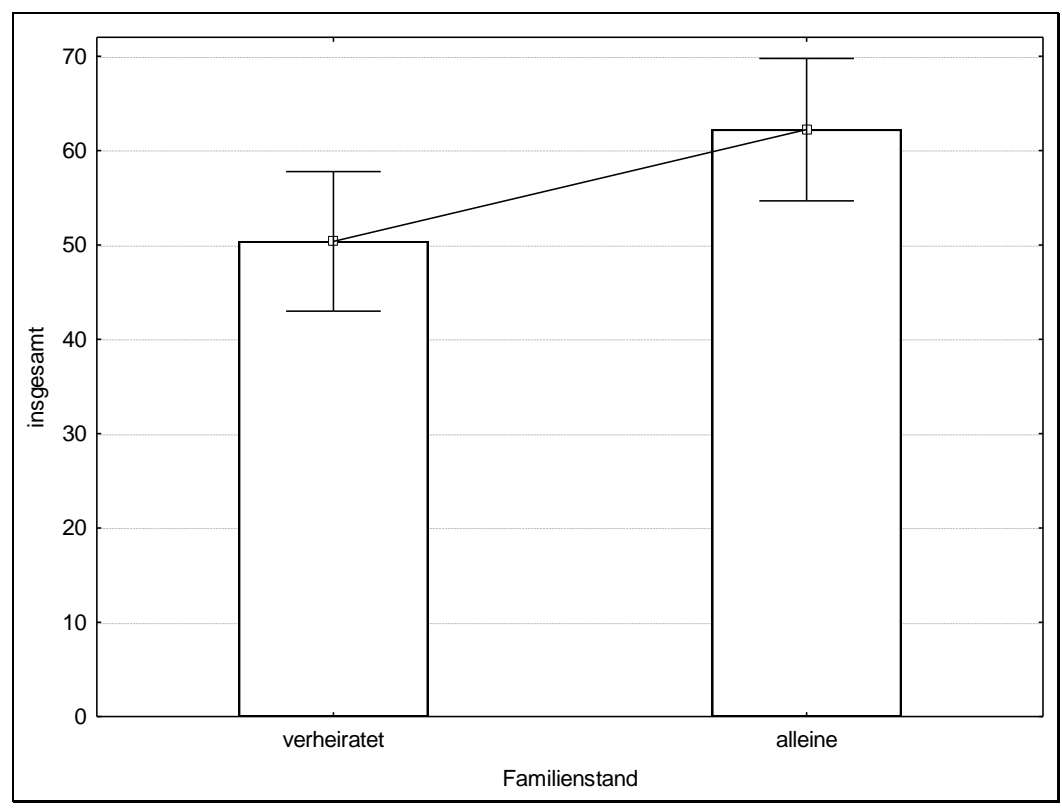

Abbildung 18: Einflussfaktor Familienstand

Dargestellt sind die Mittelwerte und die Standardabweichungen der Lebensqualität insgesamt (UW-QOL v4-Fragebogen) über den gesamten Zeitraum in Abhängigkeit vom Familienstand. Verheiratet: $M W=50,4, S D=7,2$ / Alleine: $M W=62,2, S D=7,2$ 


\subsubsection{Einflussfaktor Religion}

Bei der Auswertung des UW-QOL v4-Fragebogens nach Zugehörigkeit zu einer Religion oder hinsichtlich der Angabe konfessionslos ist kein signifikanter Unterschied zwischen den Gruppen $(p=0,908)$ festzustellen.

In der vorliegenden Untersuchung wiesen die konfessionszugehörigen Patienten eine vergleichbare Lebensqualität auf wie die Atheisten (Abbildung 19).

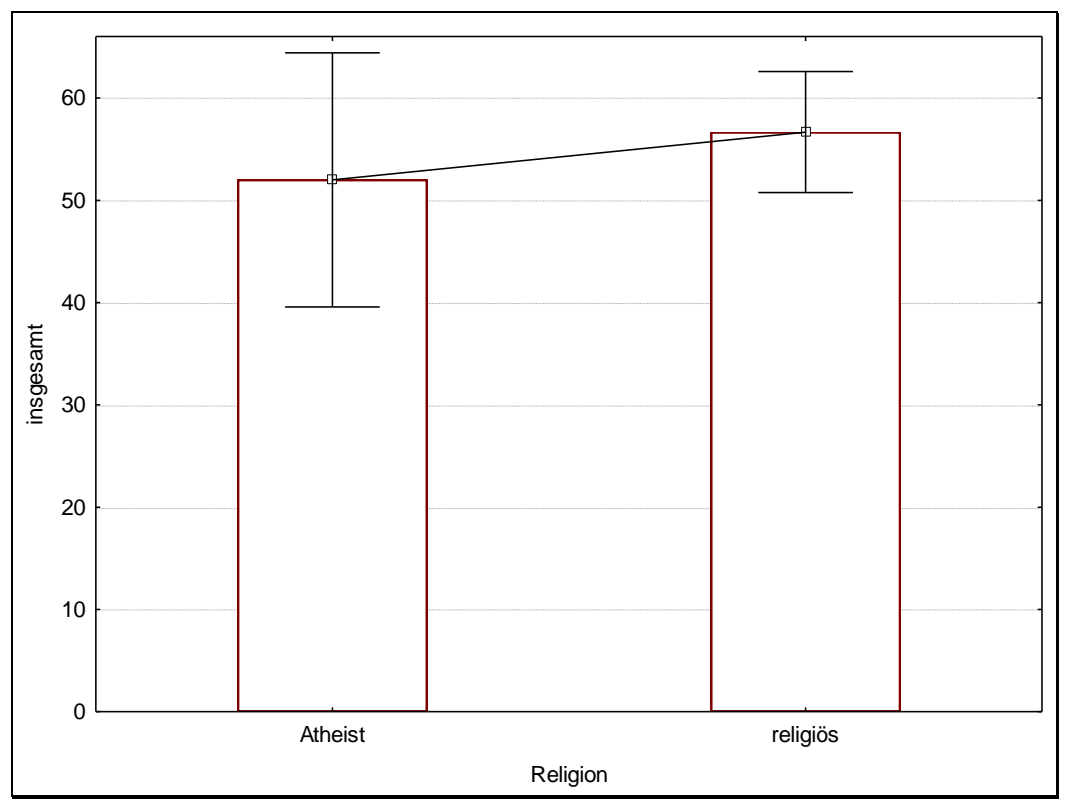

Abbildung 19: Einflussfaktor Religion

Dargestellt sind die Mittelwerte und die Standardabweichungen der Lebensqualität (UW-QOL v4-Fragebogen) über den gesamten Zeitraum in Abhängigkeit von Konfessionszugehörigkeit. Atheist: $M W=52, S D=11,6 /$ Religiös: $M W=56,7, S D=5,9$ 


\subsubsection{Detaillierte Untersuchung der physischen Funktion}

Eine differenziertere Evaluation der Lebensqualität wird durch die getrennte Darstellung der Verläufe für die physische und die sozial-emotionale Funktion des UW-QOL v4Fragebogens ermöglicht. Der Bereich physische Funktion setzt sich aus den Fragen zu Aussehen, Schlucken, Kauen, Sprache, Geschmack und Speichel zusammen. Wie bereits oben detailliert beschrieben, konnte man ein signifikantes Ergebnis für die Globalanalyse der physischen Funktion $(\mathrm{p}<0,05)$ erkennen.

Es resultierten folgende p-Werte bei der Globalanalyse der Subfunktionen: Aussehen $(0,318)$, Schlucken $(\mathbf{0 , 0 4 5})$, Kauen $(\mathbf{0 , 0 0 5})$, Sprache $(\mathbf{0 , 0 3 0})$, Geschmack $(\mathbf{0 , 0 3 2})$, Speichel (0,352). In der folgenden Abbildung 20 sind nun die einzelnen Subfunktionen der physischen Funktion in Abhängigkeit von der Zeit dargestellt. Die Bewertung der physischen Funktion (y-Achse) ist im zeitlichen Verlauf von präoperativ bis 360 Tage postoperativ veranschaulicht (x-Achse). Eine Steigung der Kurve kennzeichnet eine Verschlechterung der physischen Lebensqualität. Einheitlich im Verlauf der sechs Kurvenverläufe ist der deutliche Anstieg der Mittwerte von prä- auf postoperativ zu konstatieren, der sich im Anstieg der Kurve widerspiegelt. Im Bereich der physischen Funktion zeigt Kauen die höchsten Mittelwerte im zeitlichen Verlauf (Abbildung 20). Auffällig ist der starke Anstieg der Funktion Kauen von prä- zu postoperativer Lebensqualität (von 16 auf 43).

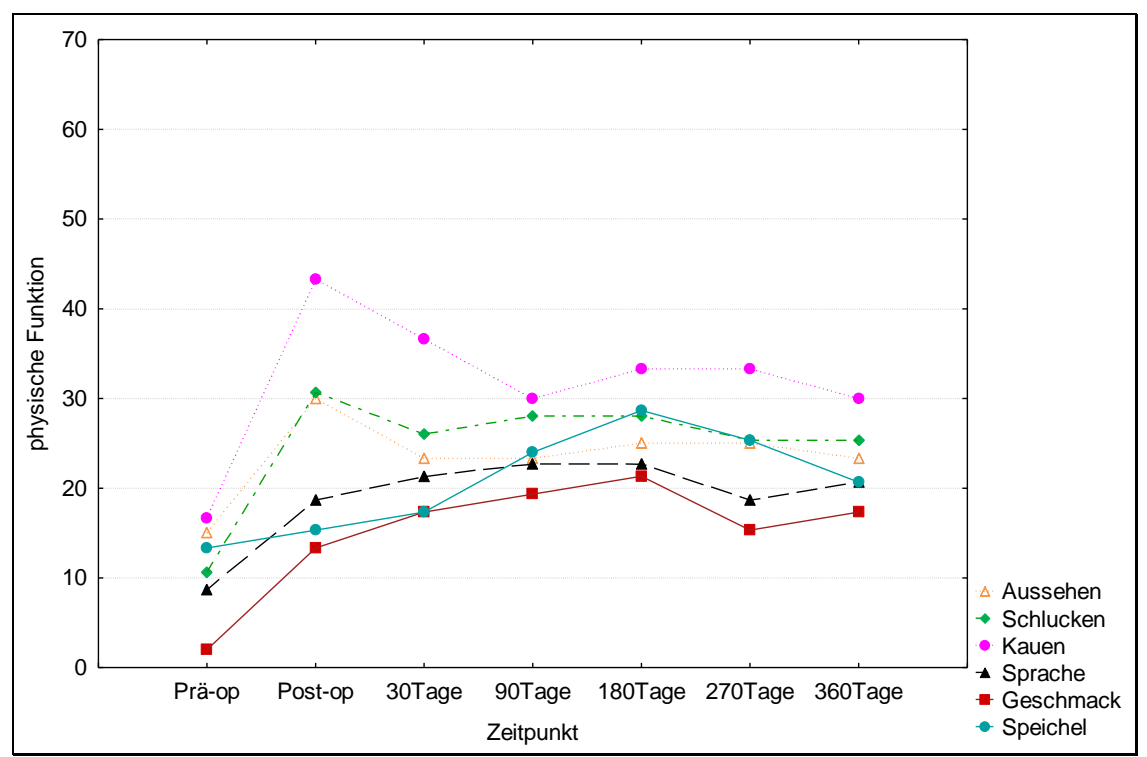

Abbildung 20: Physische Funktion im zeitlichen Verlauf

Dargestellt sind die zeitlichen Verläufe der Mittelwerte von Aussehen, Schlucken, Kauen, Sprache, Geschmack und Speichel (physische Subfunktionen des UW-QOL v4-Fragebogens) über einen Zeitraum von prä-op bis 360 Tage post-op.

Hohe Werte in der Abbildung bedeuten eine schlechte, niedrige Werte dagegen eine gute Lebensqualität. Mittelwerte und Standardabweichungen sind in Tabelle 15 (S 80) zu finden. 
In den folgenden Tabellen 9-11 sind die einzelnen Zeitpunktvergleiche für die Funktionen Kauen, Schlucken und Geschmack aufgelistet. Die Funktionen Aussehen und Speichel sind deshalb nicht aufgeführt, da die Globalanalyse einen $p$ Wert >0,05 ergab.

Tabelle 9: p-Werte Kauen

Die Zeitpunktvergleiche für Kauen ergaben folgende p-Werte:

\begin{tabular}{|l|l|l|l|l|l|l|l|}
\hline & Prä-op & Post-op & $\begin{array}{l}30 \\
\text { Tage }\end{array}$ & $\begin{array}{l}90 \\
\text { Tage }\end{array}$ & $\begin{array}{l}180 \\
\text { Tage }\end{array}$ & $\begin{array}{l}270 \\
\text { Tage }\end{array}$ & $\begin{array}{l}360 \\
\text { Tage }\end{array}$ \\
\hline Prä-op & & & & & & & \\
\hline Post-op & $<\mathbf{0 , 0 0 1}$ & & & & & & \\
\hline 30 Tage & $<\mathbf{0 , 0 0 1}$ & 0,1626 & & & & & \\
\hline 90 Tage & $\mathbf{0 , 0 3 6 6}$ & $\mathbf{0 , 0 4 0 3}$ & 0,3341 & & & & \\
\hline 180 Tage & $\mathbf{0 , 0 1 3 9}$ & 0,0824 & 0,5811 & 0,3384 & & & \\
\hline 270 Tage & $\mathbf{0 , 0 1 3 9}$ & 0,0824 & 0,5811 & 0,3384 & 1,0000 & & \\
\hline 360 Tage & 0.0993 & 0,1035 & 0,3341 & 1,0000 & 0,3331 & 0,5811 & \\
\hline
\end{tabular}

Tabelle 10: p-Werte Sprache, Schlucken

Die Zeitpunktvergleiche für Sprache und Schlucken ergaben folgende p-Werte:

\begin{tabular}{|l|l|l|l|l|l|l|l|}
\hline & Prä-op & Post-op & $\begin{array}{l}30 \\
\text { Tage }\end{array}$ & $\begin{array}{l}90 \\
\text { Tage }\end{array}$ & $\begin{array}{l}180 \\
\text { Tage }\end{array}$ & $\begin{array}{l}270 \\
\text { Tage }\end{array}$ & \begin{tabular}{l} 
Tage \\
\hline Prä-op
\end{tabular} \\
\hline Post-op & $\mathbf{0 , 0 3 5 9}$ & & & & & & \\
\hline 30 Tage & $\mathbf{0 , 0 3 4 2}$ & 0,5530 & & & & & \\
\hline 90 Tage & $\mathbf{0 , 0 1 8 5}$ & 0,7262 & 0,3343 & & & & \\
\hline 180 Tage & $\mathbf{0 , 0 1 8 5}$ & 0,7562 & 0,7102 & 1,0000 & & & \\
\hline 270 Tage & $\mathbf{0 , 0 1 7 1}$ & 0,5351 & 0,8849 & 0,5117 & 0,6165 & & \\
\hline 360 Tage & $\mathbf{0 , 0 1 7 1}$ & 0,5647 & 0,9077 & 0,6165 & 0,5117 & 1,0000 & \\
\hline
\end{tabular}


Tabelle 11: p-Werte Geschmack

Die Zeitpunktvergleiche für Geschmack ergaben folgende p-Werte:

\begin{tabular}{|l|l|l|l|l|l|l|l|}
\hline & Prä-op & Post-op & $\begin{array}{l}30 \\
\text { Tage }\end{array}$ & $\begin{array}{l}90 \\
\text { Tage }\end{array}$ & $\begin{array}{l}180 \\
\text { Tage }\end{array}$ & $\begin{array}{l}270 \\
\text { Tage }\end{array}$ & \begin{tabular}{l} 
Tage \\
\hline Prä-op
\end{tabular} \\
\hline Post-op & $\mathbf{0 , 0 3 4 2}$ & & & & & & \\
\hline 30 Tage & $\mathbf{0 , 0 3 4 2}$ & 0,1643 & & & & & \\
\hline 90 Tage & $\mathbf{0 , 0 2 9 2}$ & 0,1887 & 0,6702 & & & & \\
\hline 180 Tage & $\mathbf{0 , 0 1 6 1}$ & 0,1038 & 0,4332 & 0,6702 & & & \\
\hline 270 Tage & 0,3343 & 0,3343 & 0,5816 & 0,3343 & 0,7262 & & \\
\hline 360 Tage & $\mathbf{0 , 0 5 0 0}$ & 0,5501 & 1,0000 & 0,7451 & 0,4149 & 0,6554 & \\
\hline
\end{tabular}

In Tabelle 15 (S. 79) sind die Mittelwerte und Standardabweichungen für die sechs Subfunktionen der physischen Funktion zu drei Zeitpunkten aufgeführt. Die Reihenfolge der Subskalen von links nach rechts entspricht gleichzeitig der Reihenfolge der absteigenden Mittelwerte. Das Kauen wurde, wie oben bereits erwähnt, am schlechtesten bewertet. Es folgen Schlucken, Aussehen, Speichel, Sprache und Geschmack.

\subsubsection{Detaillierte Untersuchung der sozial-emotionalen Funktion}

In der folgenden Abbildung sind die Subfunktionen Schmerz, Aktivität, Erholung, Schulter, Stimmung und Angst veranschaulicht. Die Mittelwerte sind über den Zeitraum präoperativ bis 360 Tage postoperativ dargestellt. Es sind deutliche Unterschiede hinsichtlich der Höhe der Mittelwerte zwischen den Subfunktionen zu erkennen.

Die Globalanalysen ergaben folgende p-Werte: Schmerz $(0,438)$, Aktivität $(0,287)$,

Erholung (0,726), Schulter (0,004), Stimmung (0,755), Angst $(0,636)$. 


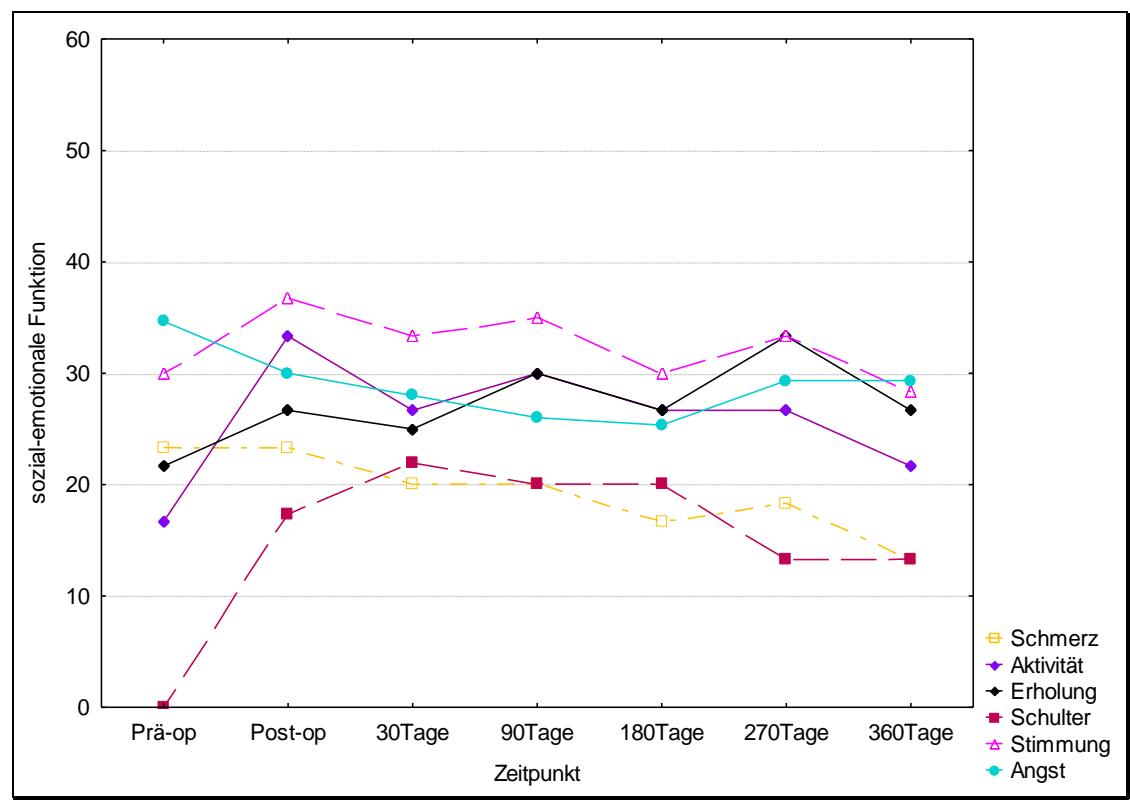

Abbildung 21: Sozial-emotionale Funktion im zeitlichen Verlauf

Zeitliche Verläufe der Mittelwerte der Bereiche Schmerz, Aktivität, Schulter, Stimmung, Angst und Erholung (sozial-emotionale Subfunktionen des UW-QOL v4-Fragebogens) über einen Zeitraum von prä-op bis 360 Tage post-op. Die Mittelwerte und Standardabweichungen sind in Tabelle 16 (S. 79) zu finden.

In Tabelle 12 sind die einzelnen Zeitpunktvergleiche für die Funktion Schulter dargestellt. Die Funktionen Schmerz, Aktivität, Erholung, Stimmung und Angst sind deshalb nicht aufgeführt, da aus der Globalanalyse ein $\mathrm{p}$ Wert $>0,05$ resultierte.

Tabelle 12: p-Werte Schulter

Die Zeitpunktvergleiche für Schulter ergaben folgende $\mathrm{p}$-Werte:

\begin{tabular}{|l|l|l|l|l|l|l|l|}
\hline & Prä-op & Post-op & $\begin{array}{l}30 \\
\text { Tage }\end{array}$ & $\begin{array}{l}90 \\
\text { Tage }\end{array}$ & $\begin{array}{l}180 \\
\text { Tage }\end{array}$ & $\begin{array}{l}270 \\
\text { Tage }\end{array}$ & $\begin{array}{l}360 \\
\text { Tage }\end{array}$ \\
\hline Prä-op & & & & & & & \\
\hline Post-op & $\mathbf{0 , 0 0 9 5}$ & & & & & & \\
\hline 30 Tage & $\mathbf{0 , 0 0 3 1}$ & 0,3263 & & & & & \\
\hline 90 Tage & $\mathbf{0 , 0 0 7 8}$ & 0,6122 & 0,3330 & & & & \\
\hline 180 Tage & $\mathbf{0 , 0 0 7 8}$ & 0,6122 & 0,3330 & 1,0000 & & & \\
\hline 270 Tage & $\mathbf{0 , 0 4 3 6}$ & 0,3321 & $\mathbf{0 , 0 2 8 1}$ & 0,0676 & 0,0676 & & \\
\hline 360 Tage & $\mathbf{0 , 0 4 3 6}$ & 0,3321 & $\mathbf{0 , 0 2 8 1}$ & 0,0676 & 0,0676 & 1,0000 & \\
\hline
\end{tabular}

Die Abbildung 21 (gleiche Seite) veranschaulicht den Kurvenverlauf für die Subfunktionen der sozial-emotionalen Funktion. Ein Anstieg der Mittelwerte, der auf eine verschlechterte 
Lebensqualität hinweist, ist vom Zeitpunkt prä- zu postoperativ erkennbar (Stimmung, Aktivität, Erholung, Schulter).

Die Aspekte Angst und Stimmung wurden von den Patienten mit der höchsten Punktzahl und damit am schlechtesten bewertet. Postoperativ beurteilten die Patienten ihre Stimmung schlechter (37 Punkte) als präoperativ (30 Punkte), was an dem Anstieg der Kurve zu erkennen ist. Die Angst unterliegt allerdings keinem Anstieg von prä- zu postoperativ, sondern sie wird bereits vom Zeitpunkt der Diagnose an als verschlechtert empfunden (35 Punkte). Sie zeigt bei postoperativ einen Abfall im Kurvenverlauf und unterliegt dann bis 360 Tage post-op minimalen Schwankungen. Ähnlich, wenn auch mit deutlich niedrigeren Mittelwerten, stellt sich der Verlauf für die Subfunktion Schmerz dar. Hier ist im Gegensatz zur Angst aber kein Anstieg von 270 auf 360 Tage zu beobachten. Besonders auffällig ist der Kurvenverlauf hinsichtlich der Ergebnisse von Aktivität und Schulter.

Die Veränderung der Subfunktion Schulter ist durch die Operationsmethode zu erklären, bei der Halslymphknoten entfernt werden. In beiden Fällen ist ein sehr deutlicher Anstieg von prä- zu postoperativ erkennbar. Fokussiert man den Verlauf dieser beiden Kurven, dann sind minimale Schwankungen über den Zeitraum von 30 bis 360 Tagen zu erkennen. 360 Tage postoperativ sinken die beiden Kurvenverläufe wieder.

Wie bereits oben erwähnt und in der Tabelle 12 mit den einzelnen Zeitpunktvergleichen aufgeführt, erbrachte lediglich die Funktion Schulter bei der Globalanalyse ein signifikantes Ergebnis $(\mathrm{p}=0,004)$

\subsubsection{Einfluss der gewählten Therapie auf die Lebensqualität}

Die Patienten der Untersuchungsgruppe befanden sich bei der Erstdiagnose in den Stadien I bis IV. Die Gruppierung zur Auswertung erfolgte, wie in Material und Methode beschrieben, in T1-T2, T3-T4 und hinsichtlich der Art der angewandten Therapie.

$\mathrm{Zu}$ differenzieren war eine freie von einer lokalen Lappenrekonstruktion. Bei zwei Patienten wurde keine Rekonstruktion, sondern nur ein primärer Wundverschluss vorgenommen. Weiterhin wurde untersucht, inwieweit die adjuvante Radiotherapie einen Einfluss ausübte. Aufgrund der geringen Fallzahl n=15 wurde hier auf eine statistische Auswertung verzichtet und die Ergebnisse nur als Zusammenfassung dargestellt.

Patienten mit T1-T2-Tumoren, freien Lappen und keiner Radiotherapie waren durchschnittlich durch die schlechtesten Werte im Bereich der Lebensqualität gekennzeichnet. Patienten (T1-T2), die eine Rekonstruktion mit lokalem Lappen erhielten, gaben im Vergleich niedrigere Werte im Lebensqualität-Fragebogen an, was einer besseren Lebensqualität entspricht. 
Bei den T1-T2-Tumoren wurde die sozial-emotionale Funktion, verglichen mit der physischen Funktion, unabhängig von der Art der Therapie als schlechter bewertet. Bei den Patienten ohne Lappenrekonstruktion und Radiotherapie konnte kein großer Unterschied zwischen der physischen und sozial-emotionalen Therapie konstatiert werden. Patienten mit T3-T4-Tumoren bewerteten ihre physische Funktion im Gegensatz zu den anderen Gruppen als schlechter. Dagegen ist bei den anderen drei Untersuchungsgruppen erkennbar, dass die sozial-emotionale Funktion als minimal schlechter beurteilt wurde.

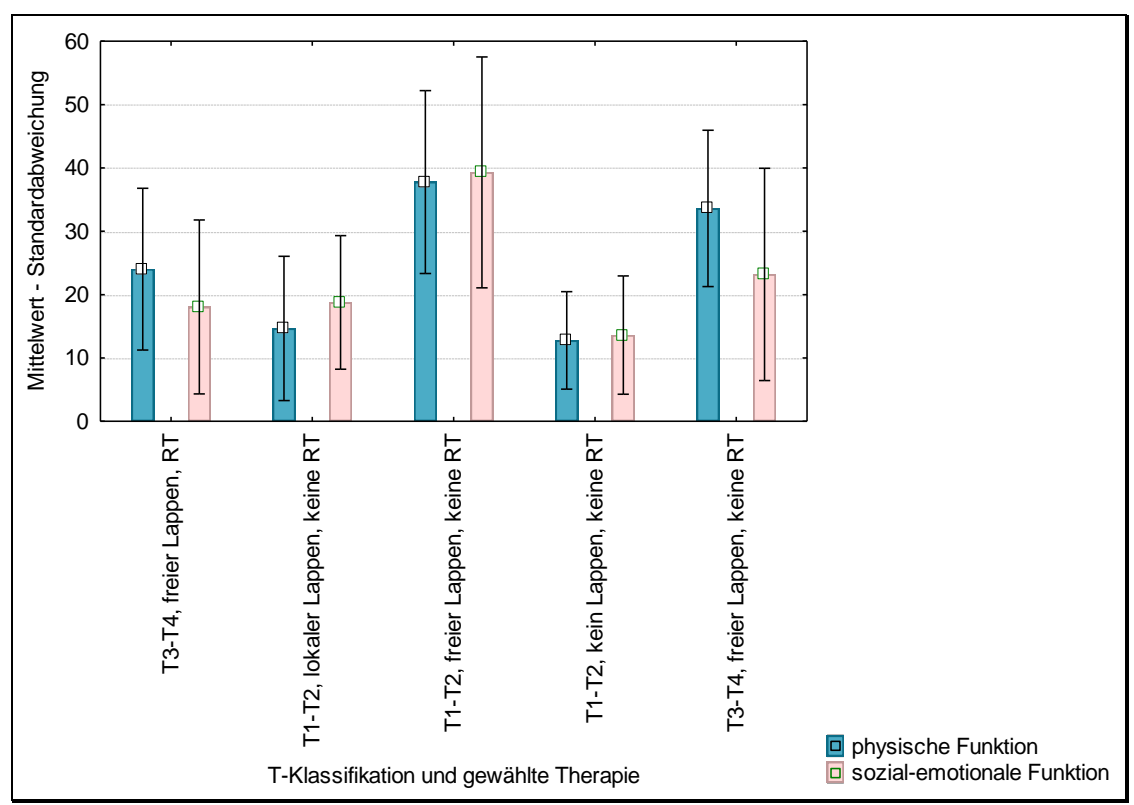

Abbildung 22: LQ / Tumorgröße, Operationsart und Radiotherapie Dargestellt sind die Mittelwerte und die Standardabweichungen von physischer und sozial-emotionaler Funktion des UW-QOL v4Fragebogens in Abhängigkeit von der Tumorgröße und der Operationsart und Radiotherapie. Von links nach rechts bestehen die Gruppen aus $\mathrm{N}=2,5,4,2,2$. Aufgrund der geringen Fallzahl und der ungleichen Verteilung wird hier auf eine statistische Auswertung verzichtet und die Ergebnisse nur als Übersicht dargestellt.

\subsubsection{Auswertung der Wichtigkeitsfragen}

Jedem Patienten wurde in dem Fragebogen zur Lebensqualität die Möglichkeit eröffnet, die drei wichtigsten Faktoren in der vergangenen Woche anzugeben.

Im Rahmen des einjährigen Monitorings wurde herausgefunden, dass Angst, Kauen und Stimmung von den Patienten am häufigsten angekreuzt wurden. 5 der 15 Patienten beurteilten präoperativ (33\%) den Faktor Kauen als am wichtigsten. Postoperativ kreuzten 6 Patienten den Faktor Kauen an. Für einen besseren Überblick wurden allerdings alle postoperativen Zeitpunkte bis 360 Tage aggregiert und zum Vergleich neben dem präoperativen Wert dargestellt (Abbildung 23). So ergibt sich im postoperativen Verlauf (33\%) keine 
Zunahme im Bereich Kauen. Präoperativ nahmen 6 Patienten (40\%) die Angst als besonders gravierend wahr, während diese postoperativ bis 360 Tage von den Patienten deutlich seltener angekreuzt wurde (14\%). Die Stimmung war für fünf Patienten (33\%) präoperativ ein wichtiger Faktor. Im postoperativen Verlauf nahm der Faktor Stimmung nicht ab (33\%). Angst und Stimmung sind Bestandteile der sozial-emotionalen Funktion, während Kauen zur physischen Funktion gehört. Bei der getrennten Auswertung der sozialemotionalen und physischen Funktion (S. 48 Abbildung 15) wurde bereits nachgewiesen, dass die sozial-emotionale Funktion von den Patienten im zeitlichen Verlauf im Durchschnitt schlechter bewertet wurde.

Die Funktion Schlucken wurde präoperativ von vier Patienten (27\%), postoperativ von fünf und nach 360 Tagen von drei als wichtig ausgewählt. Insgesamt bedeutet das eine Abnahme im postoperativen Verlauf (22\%). Erholung spielte für zwei der 15 Patienten (13\%) präoperativ eine wichtige Rolle. Im postoperativen Verlauf ist eine deutliche $\mathrm{Zu}$ nahme des Faktors Erholung erkennbar (33\%). Aktivität und Aussehen wurde präoperativ jeweils von drei Patienten (20\%) ausgewählt. Aktivität wurde postoperativ als wichtiger empfunden (28\%), während der Faktor Aussehen seltener ausgewählt wurde (19\%).

Der Faktor Schmerz wurde präoperativ von vier Patienten (27\%) als besonders wichtig erachtet. Im postoperativen Verlauf ist hier keine Veränderung zu erkennen (27\%). Schulter und Speichel wurden präoperativ von einem Patienten (7\%) ausgewählt. In beiden Fällen erfolgte postoperativ eine Zunahme (11\%).

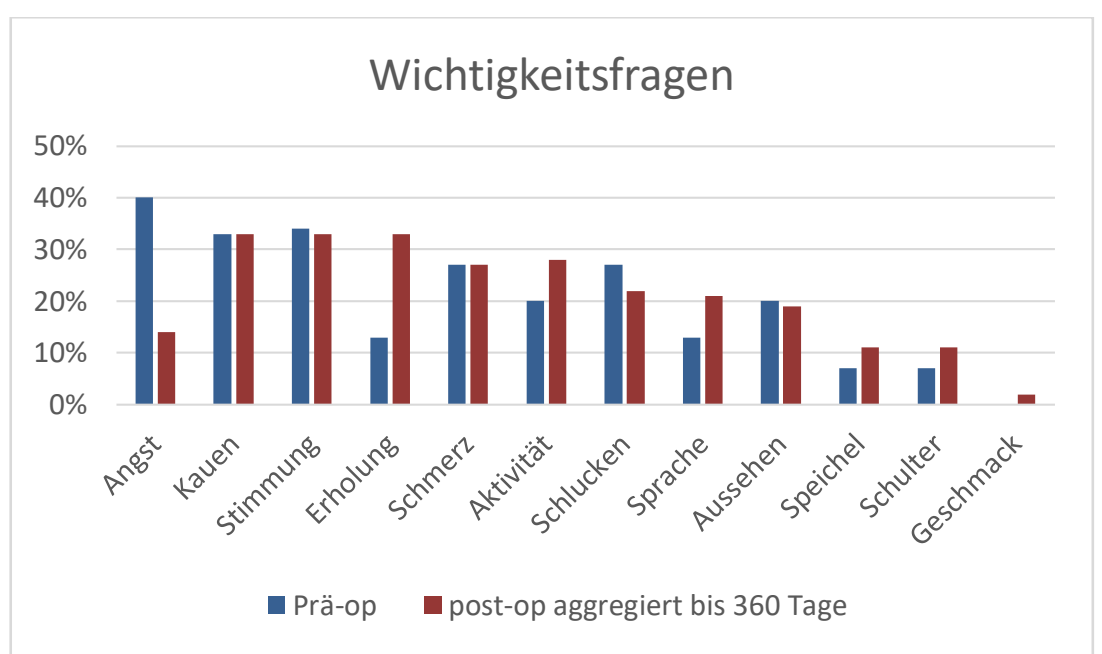

Abbildung 23: Wichtigkeitsfragen

Darstellung der Wichtigkeitsfragen in Prozent. Es erfolgte eine Gegenüberstellung präoperativ und postoperativ aggregiert bis 360 Tage. Die Patienten sollten bis zu drei Punkte ankreuzen, die für sie innerhalb der letzten Woche am wichtigsten erschienen. 


\section{Vergleich mit der Zeit vor der Krebserkrankung:}

Auf die Frage: „Wie geht es ihnen jetzt, verglichen mit den Monaten vor der Krebserkrankung?", gaben präoperativ $40 \%$ an, dass es ihnen viel schlechter geht.

Im postoperativen Verlauf wurde dies deutlich seltener empfunden (18\%).

Etwas schlechter beurteilten präoperativ $27 \%$ und postoperativ $36 \%$ ihre Lebensqualität. Als ungefähr gleich bewerteten präoperativ 33\% und postoperativ 32\% ihre Lebensqualität der letzten sieben Tage. Präoperativ empfand kein Patient seine Lebensqualität als viel oder etwas besser, postoperativ hingegen insgesamt $5 \%$.

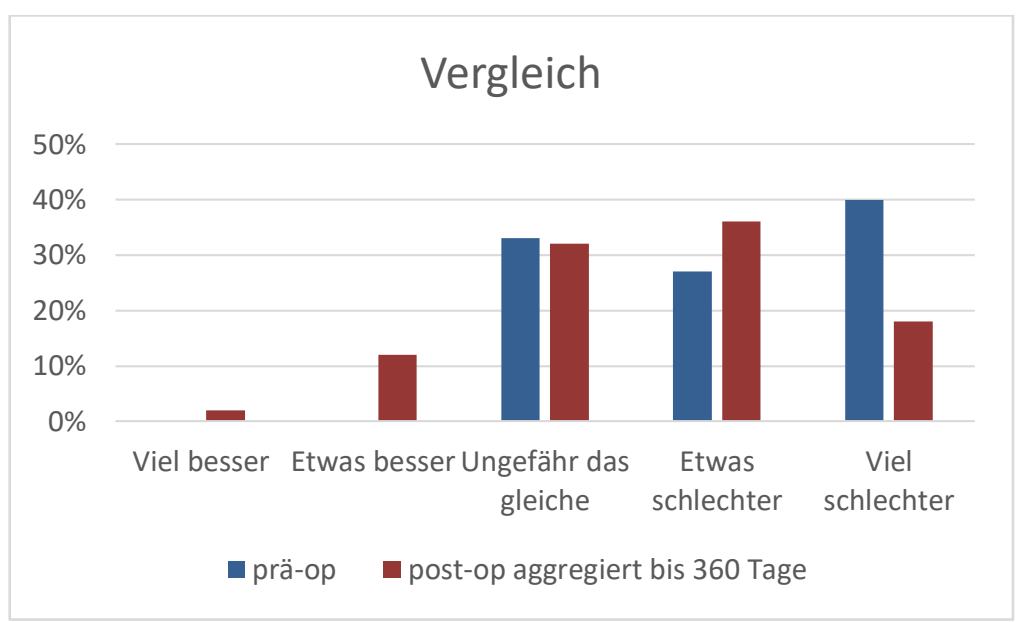

Abbildung 24: Vergleich

Darstellung der empfundenen Lebensqualität im Vergleich zu den Monaten vor der Krebserkrankung. Vorgegeben waren fünf Antwortmöglichkeiten. Abgebildet sind die Ergebnisse in Prozent von prä-op und post-op aggregiert bis 360 Tage.

Beurteilung gesundheitsbezogene Lebensqualität:

Die Beurteilung der „Gesundheitsbezogenen Lebensqualität“ in den letzten 7 Tagen wurde wie folgt bewertet. Fünf Patienten bewerteten diese präoperativ als gut (33\%) und fünf als zufriedenstellend (33\%). Drei der 15 Patienten empfanden sie als schlecht (20\%) und zwei als sehr gut (13\%). Postoperativ empfanden 38\% der Patienten ihre „Lebensqualität“ als gut, $36 \%$ als zufriedenstellend, $21 \%$ als schlecht, $3 \%$ als sehr schlecht und $2 \%$ als sehr gut. Hervorragend $(0 \%)$ wurde zu keinem Zeitpunkt ausgewählt. 


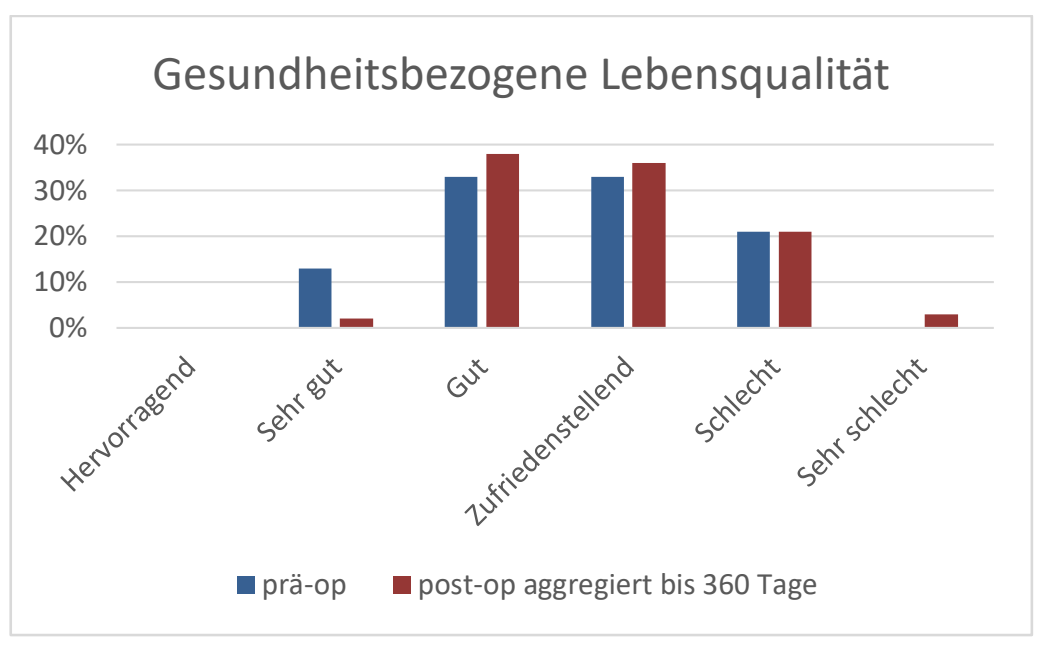

Abbildung 25: Gesundheitsbezogene Lebensqualität

Beurteilung der gesamten Lebensqualität innerhalb der letzten 7 Tage. Vorgegeben waren fünf Antwortmöglichkeiten. Abgebildet sind die Ergebnisse in Prozent von prä-op und post-op aggregiert bis 360 Tage.

Beurteilung der gesamten Lebensqualität:

Bei der Bewertung der gesamten Lebensqualität (Gesundheit, Familie, Beruf, Freunde) präoperativ nahmen fünf Patienten sie als gut wahr (33\%), vier als zufriedenstellend (27\%), drei als sehr gut (20\%), zwei als hervorragend (13\%), einer als schlecht (7\%) und keiner als sehr schlecht (0\%). Postoperativ bewerteten $40 \%$ ihre gesamte Lebensqualität als gut, $37 \%$ als gut, $18 \%$ als schlecht, $1 \%$ als hervorragend und kein Patient als sehr schlecht $(0 \%)$.

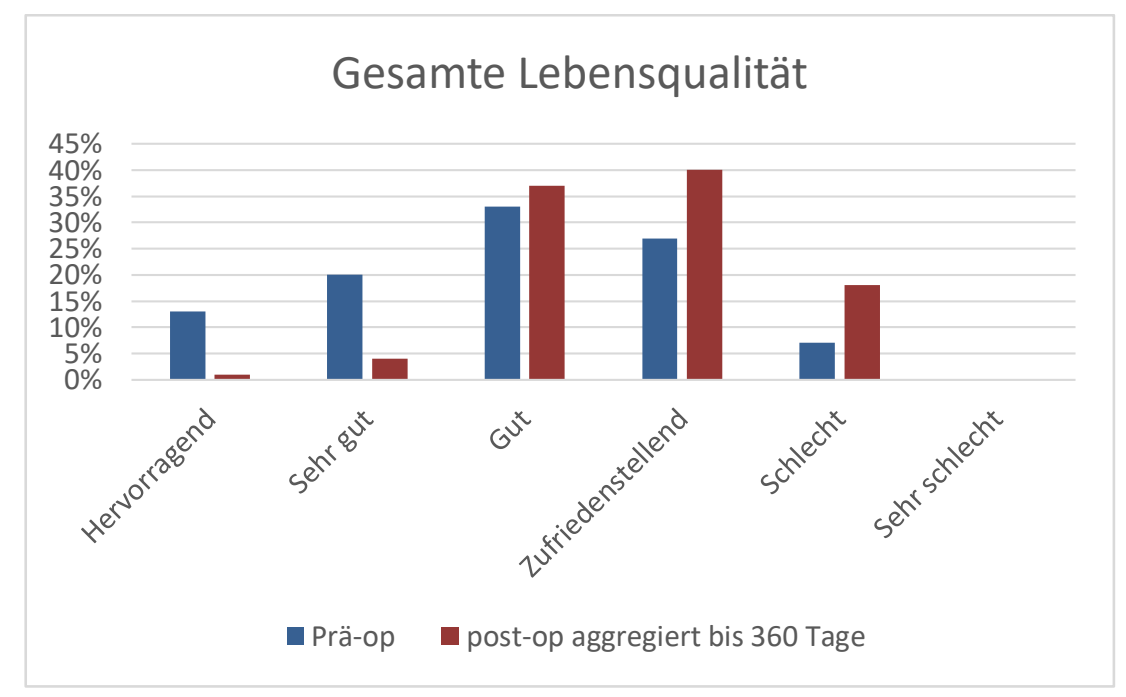

Abbildung 26: Gesamte Lebensqualität

Beurteilung der gesamten Lebensqualität innerhalb der letzten 7 Tage. Vorgegeben waren fünf Antwortmöglichkeiten. Abgebildet sind die Ergebnisse in Prozent von prä-op und post-op aggregiert bis 360 Tage. 


\subsection{Zusammenhang zwischen Posttraumatischer Reifung und Lebensqualität}

Im Folgenden soll eine Überprüfung auf einen möglichen Zusammenhang zwischen der Lebensqualität der Patienten und ihrer wahrgenommenen persönlichen Posttraumatischen Reifung vorgenommen werden. In dem angezeigten Korrelogramm, S. 64 Abbildung 27, sind die Ergebnisse veranschaulicht. Das Korrelogramm ist mit einer Farbskala dargestellt und zeigt einen Farbverlauf von Blau mit der Skalierung 1 über Weiß mit der Skalierung 0 bis zu Dunkelrot mit der Skalierung -1. Die Größe des Kreises soll zusätzlich Ausdruck der Korrelationsstärke sein.

Die physische und die sozial-emotionale Funktion wurden getrennt voneinander auf einen möglichen Zusammenhang zur Posttraumatischen Reifung untersucht. Es konnten negative Korrelationskoeffizienten festgestellt werden, womit, daraus folgend, auch ein negativer Zusammenhang für PTR und Lebensqualität resultiert. Bei hohen Werten im Bereich der Lebensqualität, die einer schlechten Lebensqualität entsprechen, nahmen die Werte im Bereich der PTR ab. Kurz zusammengefasst, bedeutet dies weniger PTR bei schlechter Lebensqualität. Allerdings ist die Stärke des Zusammenhangs als nur gering zu bewerten. Die Korrelationskoeffizienten sind der Tabelle 14, S. 64 zu entnehmen.

Bei der Überprüfung auf Signifikanz beim Zusammenhang zwischen der PTR und der Lebensqualität war nur für drei der fünf Subskalen ein $\mathrm{p}<0,05 \mathrm{zu}$ verzeichnen. Es ließen sich signifikante Zusammenhänge zwischen der physischen und der sozial-emotionalen Lebensqualität und den Skalen „Neue Möglichkeiten“, „Wertschätzung des Lebens“ und „Persönliche Stärke“ nachweisen (S. 64 Tabelle 13).

Von den fünf Subskalen der PTR sind diese drei genannten Subskalen durch die negativsten Korrelationskoeffizienten gekennzeichnet und daher sind sie mit einem größeren Kreis und einer stärkeren Färbung dargestellt.

Der p-Wert für die Korrelation von physischer Funktion und „Neue Möglichkeiten“ ist hochsignifikant. Für „Wertschätzung des Lebens“ und „Persönliche Stärke“ ist $p<0,05$ und somit ebenfalls signifikant. Bei „Religiöse Veränderungen“ und „Persönliche Beziehungen“ war kein signifikanter Zusammenhang ( $>>0,05)$ festzustellen. Die Überprüfung der Korrelation von emotionaler Funktion und den Subskalen „Wertschätzung des Lebens“, „Neue Möglichkeiten“ und „Persönliche Stärke“ ergab in allen drei Fällen ein $p<0,05$. Bei „Religiöse Veränderungen“ und „Persönliche Beziehungen“ ist kein signifikanter Zusammenhang $(p>0,05)$ zu konstatieren. 
Tabelle 13: p-Werte / Zusammenhang PTR und LQ

\begin{tabular}{|l|l|l|}
\hline & Sozial-emotionale Funktion & Physische Funktion \\
\hline Wertschätzung des Lebens & $\mathbf{0 , 0 2 0}$ & $<\mathbf{0 , 0 0 1}$ \\
\hline Neue Möglichkeiten & $\mathbf{0 , 0 3 1}$ & $\mathbf{0 , 0 2 6}$ \\
\hline Persönliche Stärke & $\mathbf{0 , 0 3 3}$ & $\mathbf{0 , 0 3 2}$ \\
\hline Persönliche Beziehungen & 0,062 & 0,057 \\
\hline Religiöse Veränderungen & 0,053 & 0,045 \\
\hline
\end{tabular}

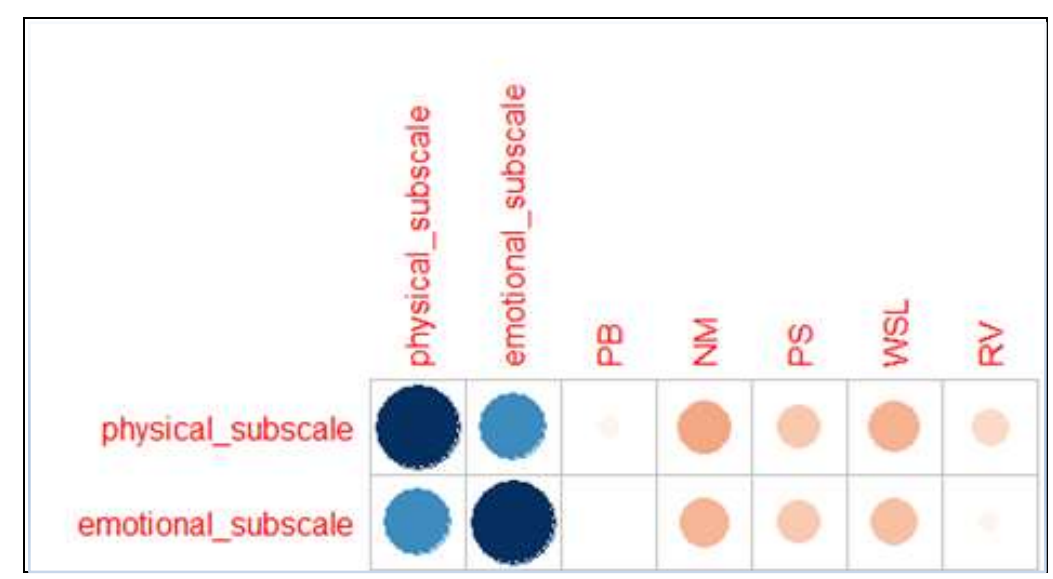

Abbildung 27: Korrelogramm

Korrelogramm zur Darstellung des Zusammenhangs zwischen Lebensqualität (sozial-emotional und physische Funktion) und der PTR. Veranschaulicht werden die Korrelationsstärken in Form von Größe und Farbintensität. Die genaue Beschreibung ist auf S. 67 zu entnehmen. $\mathrm{PB}=$ Persönliche Beziehungen, $\mathrm{NM}=$ Neue Möglichkeiten, $\mathrm{PS}=$ Persönliche Stärke, WSL = Wertschätzung des Lebens, $\mathrm{RV}=$ Religiöse Veränderungen

Tabelle 14: Korrelationskoeffizienten

\begin{tabular}{|l|l|l|}
\hline & Sozial-emotionale Funktion & Physische Funktion \\
\hline Wertschätzung des Lebens & $-0,30$ & $-0,35$ \\
\hline Neue Möglichkeiten & $-0,33$ & $-0,39$ \\
\hline Persönliche Stärke & $-0,25$ & $-0,26$ \\
\hline Persönliche Beziehungen & $-0,01$ & $-0,05$ \\
\hline Religiöse Veränderungen & $-0,05$ & $-0,19$ \\
\hline
\end{tabular}




\section{DISKUSSION}

Aktuell liegt eine Vielzahl von Studien zur posttraumatischen Entwicklung von Brustkrebspatientinnen vor. Dagegen fehlen vergleichbare Daten von Patienten mit Kopf-HalsMalignomen. Bei Studien zu dieser Tumorentität steht die postoperative Lebensqualität in Abhängigkeit von der gewählten Therapie im Vordergrund (Schliephake und Jamil 2002, Rabbels et al. 2005, Borggreven et al. 2007). Die Kopf-Hals-Malignome sind nach wie vor durch eine schlechte Überlebensrate gekennzeichnet und gehen fast immer mit einer stark verminderten Lebensqualität einher (Borggreven et al. 2007, Maschmeyer et al. 2011). Über die Ursachen für das fehlende wissenschaftliche Interesse an der PTR bei Patienten mit Kopf-Hals-Malignomen lässt sich nur spekulieren. Die Schwere der Erkrankung und die zum Teil starke Einschränkung der Lebensqualität sind jedoch ausreichende Gründe, die posttraumatische Entwicklung dieser Patienten stärker in den Fokus wissenschaftlicher Untersuchungen zu rücken. Aktuell ist prinzipiell ein zunehmendes Interesse an den psychologischen Prozessen zu konstatieren, was Grund zu der Annahme gibt, dass sich in naher Zukunft die Studienlage zu dieser Thematik verbessern wird.

Das Ziel der vorliegenden Analyse war die separate Auswertung der PTR und der Lebensqualität von Patienten mit Kopf-Hals-Malignomen nach Diagnose und chirurgischer Therapie.

Darüber hinaus sollten die möglichen soziodemografischen Einflussfaktoren und der potenzielle Zusammenhang zwischen der Lebensqualität und der PTR untersucht werden.

\subsection{Ergebnisse zur Posttraumatischen Reifung}

In der vorliegenden retrospektiven Untersuchung berichteten alle Patienten $(n=15)$ postoperativ von einer persönlichen PTR. Interessant war hier die Untersuchung des zeitlichen Verlaufs. Die wahrgenommene PTR war unmittelbar nach der Diagnose (maximal 30 Tage) stärker als ein Jahr postoperativ,h. der 360 Tage Wert war stets < als der 30 Tage Wert. Die Datenlage in der Literatur zu dieser Thematik kann nicht als einheitlich bezeichnet werden. Es gibt sowohl Studien, die eine geringere PTR bei einem größeren zeitlichen Abstand zur Diagnose detektierten (Fromm et al. 1996), als auch solche Studien, in denen die PTR im postoperativen Verlauf zunahm bzw. in denen kein Zusammenhang zwischen den beiden Parametern festgestellt werden konnte (Cordova et al. 2001b, Sears et al. 2003). 
Bei der unmittelbar nach der Diagnose subjektiv verstärkt wahrgenommenen PTR kann es sich möglicherweise um eine Illusion handeln und als Selbstberuhigung im Zuge des Verarbeitungsprozesses fungieren. Die Frage nach der Objektivierbarkeit der subjektiv wahrgenommenen PTR beschäftigte schon andere Autoren und wird immer noch kontrovers diskutiert.

Während Tedeschi und Calhoun (2004) von einer objektiv stattfindenden Entwicklung ausgehen, halten andere Autoren die Entwicklung eher für eine defensive Illusion. Nach Ansicht dieser Autoren versuchen Personen, die ein Trauma durchlebt haben, einen Sinn in dem Erlebten zu finden. Dieses Bestreben kann dazu führen, dass Überlebende versuchen, sich selbst davon zu überzeugen, dass das Trauma positive Folgen hat. Diese Art der Bewältigung wird als adaptiv und funktional palliativ bewertet (Maercker 2009). Das hierzu von Zoellner und Maercker entwickelte Janus-Modell beschreibt einen durch zwei unterschiedliche Phasen gekennzeichneten Bewältigungsprozess (Zoellner und Maercker 2006). Die genaue Erläuterung des Janus-Modells ist auf den Seiten 15 und 16 zu finden.

Das Modell dient aber nicht dazu, die unterschiedlichen Bewältigungsprozesse in richtig und falsch bzw. Illusion oder Realität zu differenzieren. Maercker macht deutlich, dass eine PTR auch dann von begrenzter Dauer sein kann, wenn sie keine Pseudo-Reifung darstellt und somit Teil der Realität ist (Maercker 2009). In der vorliegenden Studie nahmen alle Patienten eine PTR wahr und in allen Fällen war das Ausmaß der wahrgenommenen PTR kurz nach der Diagnose am größten. Obwohl die Patienten in der vorliegenden Studie den zeitlichen Verlauf der PTR als relativ homogen erlebten, erweist sich die Bewertung der Qualität der PTR als schwierig. Es lässt sich nicht mit Sicherheit sagen, ob die erlebte PTR real ist oder als eine defensive Illusion zu bewerten ist. Ob eine Unterscheidung überhaupt sinnvoll ist, scheint fraglich, da beide im Janus-Modell beschriebenen Aspekte einen positiven Bewältigungsprozess darstellen. Festzuhalten gilt die Tatsache, dass die Werte im postoperativen Verlauf nicht auf 0 abfallen, sondern selbst 360 Tage nach der Diagnose noch positive Veränderungen wahrgenommen werden. Interessant wäre natürlich eine Studie über einen noch längeren Zeitraum, um den weiteren Verlauf zu beobachten.

\section{Bereiche der wahrgenommenen Posttraumatischen Reifung:}

Die von den Patienten wahrgenommenen positiven Veränderungen betrafen alle von dem PPR-Fragebogen erfassten Bereiche „Intensivierung persönlicher Beziehungen“ (PPR1), „Entdeckung neuer Möglichkeiten“ (PPR2), „Bewusstwerden eigener Stärken“ (PPR3), 
„Wertschätzung des Lebens“ (PPR4), „Religiös- spirituelle Veränderungen“ (PPR5) (Tedeschi und Calhoun 1996).

Die Patienten stellten eine starke Verbesserung im Bereich „Wertschätzung des Lebens“ $(M=4,1, S D=0,8, \mathrm{p}<0,05)$ und „Persönliche Beziehungen“ $(M=3,9, S D=0,9, \mathrm{p}<0,05)$ fest. „Religiös-spirituelle Veränderungen“ $(M=2,2, S D=1,4, \mathrm{p}>0,05)$ spielten dagegen nur eine vergleichsweise geringe Rolle. „Persönliche Stärke“ $(M=3,3, S D=0,9, \mathrm{p}<0,05)$ und „Neue Möglichkeiten“ $(M=3, S D=0,9, \mathrm{p}<0,05)$ rangierten im Mittelfeld.

Diese Ergebnisse stehen im Einklang mit den Resultaten der longitudinalen Studie von Sears et al. 2003. Bei dieser wurde der Posttraumatic Growth Inventory-Fragebogen von Tedeschi und Calhoun verwendet. „, Wertschätzung des Lebens“ ( $M=3,5, S D=1,3)$ erzielte in dieser Studie den höchsten Mittelwert, gefolgt von der Subskala „Persönliche Beziehungen“ $(M=3, S D=1,2)$. „Persönliche Stärke“ $(M=2,8, S D=1,4)$ wurde ebenfalls am drittstärksten bewertet. Im Gegensatz zu unseren Ergebnissen nahmen „Religiöse Veränderungen“" $(M=2,7, S D=0,9)$ allerdings bei den Patientinnen hier eine stärkere Position ein. Das Gefühl, über „Neue Möglichkeiten“ $(M=2,1, S D=1,4)$ zu verfügen, wurde von den Brustkrebspatientinnen am wenigsten empfunden (Sears et al. 2003). Anhand der oben genannten Ergebnisse wird also deutlich, dass die Patienten in manchen Bereichen vermehrt PTR erfahren. „Wertschätzung des Lebens“ ist nicht ohne Grund deshalb einer der wichtigsten Bereiche, da die Patienten durch die Diagnose bewusst mit einer lebensbedrohlichen Krankheit konfrontiert werden, was wiederum Auslöser für eine veränderte Sichtweise sein kann (Janoff-Bulman 2004). Das Überstehen des Traumas und das Gefühl, eine zweite Chance zu erhalten, können Anlass für den Betroffenen sein, in seinem Leben neue Prioritäten zu setzen (Tedeschi und Calhoun 1996). Von einem Überstehen des Traumas lässt sich im Falle dieser Studie allerdings nur schwer sprechen, da die Erkrankung mit einer schlechten Prognose verbunden ist und die Studie nur über einen Zeitraum von einem Jahr durchgeführt wurde. Trotzdem ist davon auszugehen, dass die Patienten neue Prioritäten setzen und ihre Ansicht in Bezug auf ihr existentes Weltbild ändern. Im Freitext gaben einige Patienten an, dass sie große Angst verspüren und dankbar sind für die Unterstützung von Familie und Freunden, durch die sich unter anderem die Intensivierung des Bereiches „Persönliche Beziehungen“" erklärt.

Es lässt sich als eine Stärkung der zwischenmenschlichen Beziehungen konstatieren (Zoellner et al. 2006). 


\section{Diagnose Krebs als Auslöser für Posttraumatische Reifung:}

Lebensbedrohliche Erkrankungen, wie Krebs, sind eines von vielen möglichen Traumata, die eine PTR auslösen können (Collins et al. 1990, Cordova et al. 2001a).

Die Diagnose Krebs verursacht einerseits Angst und Unsicherheit im Hinblick auf die persönliche Zukunft aus, andererseits resultieren aus ihr aber auch physische Folgen, mit denen der Patient zu kämpfen hat. Für die meisten Menschen löst daher die lebensbedrohliche Diagnose Krebs eine emotionale Krise aus, die durch Depressionen und Unsicherheit gekennzeichnet ist. Bislang konnte noch nicht herausgefunden werden, warum die einen Menschen nach der Diagnose eine PTR erfahren, während andere dagegen eine PTBS ausbilden. Hinsichtlich der Genese einer PTR werden soziodemografische Daten als Einflussfaktoren diskutiert (Linley und Joseph 2004).

Ebenso wie auch in anderen Studien, die sich mit PTR beschäftigen, stellte sich die Frage, ob es mögliche Faktoren gibt, die eine PTR begünstigen. Aus diesem Grund wurde die Auswertung der PPR-Fragebögen in Abhängigkeit von den Patientencharakteristika (Alter, Geschlecht, Familienstand, Konfessionszugehörigkeit) durchgeführt. Aus der Analyse des Einflusses bezüglich des Alters der Patienten auf die PTR resultierte in drei von fünf Bereichen ein höherer Mittelwert für Patienten unter 60 Jahren. Hervorzuheben ist der signifikante Zusammenhang zwischen Patienten unter 60 Jahren und der verstärkten Wahrnehmung „Neuer Möglichkeiten“ ( $M W=3,4, \mathrm{SD}=1,8, \mathrm{p}<0,05)$. Klauer (1998) nimmt an, dass ältere Menschen aufgrund vorangegangener Erlebnisse über eine andere Wahrnehmung verfügen. Der Verlust von Freunden und Verwandten lässt die eigene Krankheit in einem anderen Licht erscheinen. Die Wahrnehmung von positiven Effekten im Zuge des Bewältigungsprozesses ist dadurch gemindert. Diese Erklärungsmöglichkeit wird durch die Befunde anderer Studien unterstützt (Tempelaar et al. 1989, Lechner et al. 2003, Sears et al. 2003, Linley und Joseph 2004, Widows et al. 2005). Auch in den Bereichen „Persönliche Beziehungen“ ( $M W=3,8, S D=1,7, \mathrm{p}>0,05)$, „Persönliche Stärke“ $(M W=3, S D=1$, p>0,05) und „Religiöse Veränderungen“ wurden tendenziell höhere Werte erzielt. Es lässt sich also eine Tendenz dahin gehend feststellen, dass jüngere Patienten mehr PTR erfahren.

Es konnte kein signifikanter Zusammenhang zwischen PTR und dem Geschlecht des Patienten, dem Familienstand oder der Religionszugehörigkeit konstatiert werden. Diese Wahrnehmung galt für alle Subskalen (1. Persönliche Beziehungen 2. Persönliche Stärke 3. Wertschätzung des Lebens 4. Neue Möglichkeiten 5. Religiöse Veränderungen). Ein vor- 
wiegend nicht signifikanter Zusammenhang zwischen diesen soziodemografischen Daten und der posttraumatischen Reifung konnte in der Literatur nicht bestätigt werden. In zahlreichen Studien wurde ein Einfluss soziodemografischer Parameter eruiert (Klauer 1998, Tempelaar et al. 1989, Lechner et al. 2003, Sears et al. 2003, Linley und Joseph 2004, Widows et al. 2005, Schmidt et al. 2012, Lepore et al. 1996). Die Ergebnisse der zuvor angeführten Studien werden im weiteren Verlauf unter den einzelnen Punkten präzisiert. Trotz des Fehlens des signifikanten Zusammenhangs zwischen dem Geschlecht und der PTR zeigten Frauen in allen fünf Subskalen dieser Untersuchung tendenziell höhere Werte (S. 42 Abbildung 11). Andere Autoren gelangten zu vergleichbaren Befunden (Linley und Joseph 2004; Swickert und Hittner 2009). Ein möglicher Grund für die verstärkte PTR bei Frauen können geschlechtsspezifische Bewältigungsstrategien sein. Frauen empfinden die soziale Unterstützung und die Möglichkeit, über die Erkrankung reden zu können, als überaus wichtig. Gleichzeitig berichten diejenigen Frauen, die ausführlich über ihre Krebserkrankung sprechen, seltener von Depressionen und von einem besseren Wohlbefinden (Cordova et al. 2001b).

Der Familienstand (verheiratet vs. alleinstehend) ergab keine signifikante Korrelation zu den verschiedenen Subskalen des PPR-Fragebogens. Die Mittelwerte in vier der fünf Subskalen differieren nicht in starkem Maße, lediglich im Bereich „Religiöse Veränderungen“ gaben alleinstehende Patienten eine größere Veränderung an (S. 45 Abbildung 13). Auch wenn kein signifikanter Zusammenhang festgestellt werden konnte, ist doch anzunehmen, dass die soziale Unterstützung einen entscheidenden Faktor für den Bewältigungsprozess darstellt. Die Menschen, welche die Möglichkeit haben, sich zu öffnen und über ihre Erkrankung und Ängste zu sprechen, scheinen eine bessere posttraumatische Entwicklung zu erleben (Lepore 1997, Lepore und Revenson 2006, Swickert und Hittner 2009). Es ist daher interessant, einen intensiveren Blick auf die Bewertung der einzelnen Fragen innerhalb der Subskala „Intensivierung persönlicher Beziehungen“ zu werfen. Die Erkenntnis zu wissen, dass man sich in schweren Zeiten auf andere Menschen verlassen kann (S. 32 PPRFragebogen Frage 6), schien für die Patienten besonders wichtig zu sein und erzielte daher innerhalb dieser Subskala den höchsten Mittelwert $(M W=4,4)$. Auch in den Freitexten betonten die Patienten immer wieder, wie wichtig es ihnen ist, dass sie sich auf jemanden verlassen können und soziale Unterstützung bekommen.

Als ungünstig ist hier die Differenzierung in verheiratet und alleinstehend zu betrachten, da alleinstehend nicht notwendigerweise das Fehlen von sozialer Unterstützung zu bedeuten 
hat. Eine spezifischere Unterteilung ist anzustreben, um eine detailliertere und genauere Auswertung zu ermöglichen.

Auch die Religiosität übte in der vorliegenden Studie keinen signifikanten Einfluss auf die Ergebnisse des PPR-Fragebogens aus. Die Patienten, die sich als konfessionslos bezeichneten, erzielten in drei der fünf untersuchten Bereiche höhere Mittelwerte (S. 46 Abbildung $14)$.

Dagegen belegten andere Untersuchungen, dass stark gläubige Menschen weniger Schwierigkeiten mit der Bewältigung von Traumata zu verzeichnen haben (Linley und Joseph 2004). Eine Studie fand heraus, dass Brustkrebspatientinnen ihren Glauben als unterstützend und kraftspendend in der Zeit nach der Diagnose beurteilten (Schreiber und Brockopp 2012). Die Beschäftigung mit religiösen und fundamentalen Thematiken kann an sich als PTR empfunden werden. Auch solche Personen, die sich selbst als Atheisten bezeichnen, können eine PTR in diesem Bereich erfahren. Eine lebensbedrohliche Erkrankung kann das Interesse an der Auseinandersetzung mit existenziellen Fragen verstärken (Tedeschi und Calhoun 2004). Hier sollte allerdings über eine andere Kategorisierung nachgedacht werden, da eine fehlende Konfession nicht zwangsläufig mit Atheismus einhergehen muss.

\subsection{Ergebnisse zur Lebensqualität}

Die Auswertung der Lebensqualität in Abhängigkeit von der Zeit ergab, dass sich sowohl die physische $(M W=22,5, S D=16, \mathrm{p}<0,05)$ als auch die sozial-emotionale Funktion $(M W$ $=24,8, S D=16,4, \mathrm{p}>0,05)$ bei allen Patienten postoperativ verschlechterten.

Diese Befunde stimmen mit einer Analyse zur gesundheitsbezogenen Lebensqualität bei Patienten mit Mundhöhlenkarzinom überein, die postoperativ eine vorübergehende Minderung der physischen sowie der sozial-emotionalen Funktion ergab (Schliephake und Jamil 2002). Vergleichbare Ergebnisse, ebenfalls mit dem UW-QOL v4-Fragebogen erfasst, wurden in einer einjährigen Befragung bei Patienten mit oropharyngealen Karzinomen herausgefunden. Die longitudinale Studie wies eine signifikant schlechtere postoperative Lebensqualität nach. Die stärkste Verschlechterung hinsichtlich der Funktion konnte bei dem Vergleich der Zeitpunkte präoperativ und ein Jahr postoperativ festgestellt werden (Biazevic et al. 2010). Eine Betrachtung des prä- und postoperativen Zeitpunkts erscheint im Blick auf die vorliegenden Ergebnisse deshalb am sinnvollsten, da hier die stärksten Veränderungen wahrgenommen wurden. Auffällig ist der starke Anstieg von prä- zu post- 
operativ, der bei allen Subfunktionen, mit Ausnahme der Angst und des Schmerzes, zu beobachten ist.

Die postoperativen Mittelwerte sind die höchsten innerhalb des Untersuchungszeitraums bis zu 360 Tagen. Vom Zeitpunkt post-op bis 360 Tagen kann dann eine Abnahme der Werte, also eine Verbesserung der Lebensqualität, vermerkt werden. Allerdings konnte für keine Funktion ein ähnlicher Ausgangswert gemessen werden, was gleichbedeutend damit ist, dass die Lebensqualität von keinem Patienten nach 360 Tagen als genauso gut empfunden wurde wie zum Zeitpunkt der präoperativen Befragung. Den Kurvenverlauf kann man in den Abbildungen 20 und 21 nachvollziehen. Die Mittelwerte und Standardabweichungen der Funktionen Kauen, Schlucken, Aussehen, Speichel, Sprache, Geschmack, Stimmung, Erholung, Aktivität und Schulter sind im Anhang zu finden (Tabellen 15 und 16, S. 79).

Im Bereich physische Funktion erwiesen sich Kauen $(M W=31,9, S D=26,9, \mathrm{p}<\mathbf{0 , 0 5})$ und Schlucken $(M W=24,9, S D=25,6, \mathbf{p}<\mathbf{0 , 0 5})$ als diejenigen Faktoren, die in maßgeblicher Weise für die postoperative Verschlechterung der Lebensqualität verantwortlich waren. Es ist davon auszugehen, dass diese Ergebnisse auf die Lokalisation des Plattenepithelkarzinoms in der Mundhöhle und die in der Regel notwendige invasive Therapie zurückzuführen sind. Signifikante Verläufe konnten in der hier vorliegenden Arbeit für Kauen, Sprache und Geschmack festgestellt werden. Diese Ergebnisse stimmen mit denen einer brasilianischen Studie überein, betreffend der QOL bei Patienten mit Mundhöhlen- und Oropharynxkarzinom (Villaret et al. 2008), in welcher die Funktion Kauen in einem einjährigen Verlauf die stärkste Verschlechterung und die Globalanalyse einen signifikanten Verlauf $(\mathrm{p}<0,05)$ zeigten. Ebenso fiel in Villarets Studie bei der Funktion Angst die Besonderheit im prä- und postoperativen Verlauf auf. Die Angst war präoperativ sehr groß und nahm im postoperativen Verlauf ab. Vergleicht man dies mit den Ergebnissen der hier vorliegenden Studie, so ist zu bemerken, dass der Faktor Angst $(M W=28,9, S D=27,3$, p>0,05) für die Patienten eine ebenso große Rolle spielte und der einzige Faktor ist, der bereits präoperativ stark erhöht war und eine Reduktion des Mittelwerts von prä- zu postoperativ zeigte. Allerdings kristallisierte sich hier keine signifikante Reduktion heraus.

In den Freitexten thematisierten die Patienten neben dem Gefühl der fehlenden oder unzureichenden Aufklärung und Zuwendung durch Ärzte und Arzthelferinnen häufig auch die Angst vor der Zukunft. Dafür ein Beispiel: „Meine Lebensqualität ist durch die Diagnose Krebs vor sieben Tagen massiv beeinträchtigt. Ich habe enorme Ängste vor der OP und 
den damit verbundenen Einschränkungen. Körperlich fühle ich mich aber nicht eingeschränkt.“ In Bezug auf die Funktion Angst ist allerdings kein signifikanter Unterschied zwischen den einzelnen Messzeitpunkten ( $p>0,05)$ zu konstatieren.

Die sozial-emotionale Funktion wird neben der Angst stark durch die Stimmung $(M W=$ $32,4, S D=25,2)$ der Patienten beeinflusst, welche im gesamten Verlauf sehr hohe Mittelwerte zeigt und lediglich einigen Schwankungen unterliegt. Es ließ sich kein signifikanter Verlauf feststellen ( $p>0,05)$. Stimmung und Angst verlaufen gegenläufig, eine Bewertung dieses Faktum erweist sich allerdings deshalb als schwierig, da unklar ist, inwieweit beide voneinander abhängen

Auffällig ist der Kurvenverlauf des Faktors Schulter, der präoperativ bei 0 liegt und dann signifikant ansteigt $(M W=17,3, S D=25,2, \mathrm{p}<\mathbf{0 , 0 5})$. Bei der OP der Lymphknoten $($ Neck Dissection) tritt eine mögliche Beeinträchtigung der Innervation der Schultermuskulatur ein, sodass die postoperativen Beschwerden hiermit erklärt werden können.

Die Patienten, die den Faktor Speichel im Zeitraum von postoperativ bis 360 Tage als besonders wichtig empfanden, erhielten eine adjuvante Strahlentherapie.

Dies ist deshalb kein unerwartetes Ergebnis, da es sich hierbei um eine bekannte Nebenwirkung der Strahlentherapie im Kopf-Bereich handelt (Memtsa et al. 2017).

Eine Xerostomie schränkt die Patienten in starkem Maße ein, da sie schlechter schlucken und essen können. Häufig werden zusätzliche Präparate als Speichelersatz angewendet. Nicht immer liegt eine Hyposalivation vor, sondern allein die veränderte Zusammensetzung löst das Gefühl der Xerostomie aus.

Wirft man einen Blick auf die Wichtigkeitsfragen in Kapitel 4.3.9, so erkennt man hier noch einmal, welch großen Stellenwert der Faktor Angst bei den Patienten einnahm. Für 40\% der Patienten spielte präoperativ die Angst eine sehr große Rolle, postoperativ waren dies nur noch $14 \%$. Dementsprechend beurteilten $40 \%$ präoperativ ihre Lebensqualität als viel schlechter, postoperativ waren es nur noch 18\%. Erholung (prä-op: 13\%, post-op: 33\%) und Aktivität (prä-op: 20\%, post-op: 28\%) gewannen im Gegensatz zur Angst postoperativ an Wichtigkeit, was durch die Schwere der Erkrankung und die Folgen der Operation erklärbar ist.

Bei der Analyse der Ergebnisse zur Bewertung der gesundheitsbezogenen und der gesamten Lebensqualität fällt das Faktum auf, dass mehr als $50 \%$ der Patienten diese als ,gut“ und ,zufriedenstellend“ bewerteten. Diese Ergebnisse lassen die Vermutung zu, dass trotz 
der Reduktion der physischen Funktion, welche von den Patienten postoperativ erlebt wurde, die gesamte Bewertung multifaktoriell beeinflusst wird.

Die soziodemografischen Faktoren zeigten in dieser Untersuchung keine signifikanten Zusammenhänge, was allerdings durch die geringe Fallzahl und ungleiche Verteilung nur bedingt aussagekräftig ist. Hinsichtlich des Geschlechts lässt sich eine Tendenz dahin gehend erkennen, dass die Männer ihre gesundheitsbezogene Lebensqualität im Vergleich zu den Frauen tendenziell schlechter bewerteten $(\mathrm{p}=0,058)$, wobei das Signifikanzniveau nur knapp verfehlt wurde.

Während Rogers et al. (1998) bei Frauen eine bessere Lebensqualität feststellten, wiesen andere Untersuchungen keinen signifikanten Unterschied zwischen den Geschlechtern nach (Schliephake und Jamil 2002). Bei der Untersuchung der soziodemografischen Daten, wie Alter, Religion und Familienstand des Patienten, konnte kein signifikanter Zusammenhang mit der Lebensqualität festgestellt werden.

Es wird vermutet, dass die Inanspruchnahme der sozialen Unterstützung einen wertvollen Beitrag zur Bewältigung des Traumas leistet. Die Möglichkeit, sich anderen anvertrauen zu können, und das Gefühl von Unterstützung, Verständnis und Akzeptanz werden als wichtige Voraussetzungen für die Genese einer PTR angesehen (Tedeschi und Calhoun 2004). Die soziale Unterstützung erleichtert den Bewältigungsprozess und verringert emotionales Unwohlsein (Lepore und Revenson 2006).

Erst die Möglichkeit, Gedanken und Ängste aussprechen zu können, eröffnet den Betroffenen die Gelegenheit, den Verarbeitungsprozess abzuschließen (Lepore et al. 1996).

Soziale Beschränkungen, wie z. B. ein Mangel an Personen mit einem offenen Ohr für die Betroffenen, verstärken dagegen das Leid und die Belastung.

Aufgrund des kleinen Patientenkollektivs $(n=15)$ und der ungleichmäßigen Verteilung hinsichtlich der Tumorcharakteristika war ein Vergleich der Lebensqualität in Abhängigkeit von der Therapie nicht aussagekräftig. Auf eine Gegenüberstellung der Patienten mit T1T2 und T3-T4 Tumoren wurde daher verzichtet. Der Vergleich der Patienten mit T1-T2 Tumoren wies eine bessere Lebensqualität bei Lokalrekonstruktionen im Gegensatz zur Rekonstruktion mit freier Lappenplastik nach (Free Flap). Auch in anderen Studien korrelierten wenige weit fortgeschrittene Mundhöhlenkarzinome, nicht notwendige Rekonstruktionen mit freiem Lappen und das Fehlen einer adjuvanten Radiotherapie mit besserer physischer und psychischer Lebensqualität (Rogers et al. 2010). 
Die sozial-emotionale Funktion bei Patienten mit T1-T2-Tumoren wird, und zwar unabhängig von der Art der Therapie, als schlechter bewertet als die physische Funktion (Kapitel 4.2.4).

Bei den Patienten, bei denen keine Lappenrekonstruktion und Radiotherapie erfolgten, war der Unterschied zwischen physischer und sozial-emotionaler Funktion eher gering. Die schlechtere Bewertung der physischen Funktion durch Patienten mit T3-T4-Tumoren kann auf die stärkere Beeinträchtigung der körperlichen Funktionen, wie Sprechen, Schlucken und Kauen, zurückgeführt werden, und zwar in Abhängigkeit von der Größe des Tumors.

\subsection{Zusammenhang zwischen Lebensqualität und Posttraumatischer Reifung}

Die Ergebnisse der Lebensqualität-Fragebögen und PPR-Fragebögen wurden nicht nur getrennt voneinander ausgewertet, sondern zudem auch auf einen möglichen Zusammenhang hin überprüft (Kapitel 4.3). Generell wird angenommen, dass ein größerer Leidensdruck mit einem höheren Maß an PTR korreliert (Tomich und Helgeson 2012).

Bei der Auswertung der Lebensqualitäts- und PPR-Daten der Patienten mit Kopf-HalsMalignomen kristallisierte sich ein negativer Zusammenhang heraus. Patienten mit schlechter Lebensqualität (hohe Werte) gaben eine geringere PTR (niedrige Werte) an.

Die Annahme, dass ein gesteigertes Maß an PTR mit einer reduzierten Lebensqualität korreliert, wird durch die Ergebnisse der vorliegenden Analyse somit nicht bestätigt. Fromm et al. (1996) gehen jedoch davon aus, dass das Ausmaß der positiven Traumafolgen mit der Ernsthaftigkeit der Krankheit korreliert.

Eine schlechtere Diagnose sei mit einer größeren Hürde verbunden, sodass es wahrscheinlicher ist, dass der betroffene Patient ein positives Ergebnis in stärkerem Maße würdigt (Fromm et al. 1996). Damit müssten Patienten mit einem prognostisch ungünstigen KopfHals-Malignom eher eine PTR durchleben als Patienten mit einer prognostisch günstigeren Entität. Die Studienlage zum Zusammenhang zwischen Lebensqualität und PTR ist nicht konform. Die Analysen ergaben keine einheitlichen Ergebnisse. Helgeson et al. (2006) stellten keinen signifikanten Zusammenhang fest. Zusätzlich zu den Korrelationskoeffizienten wurde eine statistische Überprüfung durchgeführt. Der Signifikanztest belegte, dass die Korrelation von physischer und sozial-emotionaler Funktion mit der Wahrnehmung „Neuer Möglichkeiten“, „Wertschätzung des Lebens“ und „Persönliche Stärke“ bedeutend ist. Die verstärkte Wahrnehmung dieser Bereiche steht in Korrelation mit einer 
guten Lebensqualität. Nicht signifikant ist hingegen der Zusammenhang mit „Religiöse Veränderungen“ und „Persönliche Stärke“.

\subsection{Limitationen der Studie}

Der retrospektive Charakter der vorliegenden Studie limitiert die Aussagekraft der Ergebnisse. Die anonymisierten Daten wurden über einen Zeitraum von 1,5 Jahren gesammelt. Zunächst waren weniger als $50 \%$ aller Tumorpatienten überhaupt bereit, die genannten Fragebögen auszufüllen. Unvollständige Fragebögen waren für eine deskriptive Auswertung unbrauchbar und stellten ein Ausschlusskriterium dar. Dieses Vorgehen führte dazu, dass sich das Patientenkollektiv um weitere 50\% reduzierte. Da die Fragebögen routinemäßig nach dem Einverständnis ausgehändigt wurden, war eine intensive Betreuung der Patienten im Hinblick auf das Ausfüllen der Fragebögen und der Datenerhebung nicht gegeben. Zudem ist anzunehmen, dass einige Fragen für die Patienten schwer verständlich waren und die Beantwortungen aus diesem Grund nicht vorgenommen wurden. Die Art der Datenerhebung stellte einen limitierenden Faktor dar, da nur weniger als 25\% aller Tumorpatienten die Fragebögen vollständig, in regelmäßigen Abständen und über einen ausreichend langen Zeitraum ausfüllten. Es kann nicht ausgeschlossen werden, dass durch dieses Vorgehen die Patienten auch hinsichtlich der zu untersuchenden Faktoren (Lebensqualität, PTR) vorselektioniert wurden. Um die genannte Fehlerquelle zu minimieren, wäre ein assistiertes Ausfüllen der Fragebögen im Rahmen einer prospektiven Studie empfehlenswert. Grenzen im Bereich der PTR-Forschung ergeben sich auch durch die Schwierigkeit des interpersonellen Vergleichs (Kapitel 3). Diese Schwierigkeiten treten sowohl bei der Auswertung einer Studie als auch bei einem Vergleich von zwei oder mehr Studien auf. Die in dieser Studie untersuchten Patienten waren hinsichtlich des Karzinoms sowie dessen Lokalisation sehr homogen. Folgestudien sollten mit einem größeren Patientenkollektiv unter Berücksichtigung weiterer potenzieller soziodemografischer Einflussfaktoren auf die PTR durchgeführt werden. Wichtige Einflussfaktoren für das Ausmaß der PTR sind soziokulturelle Parameter, wie das soziale Umfeld und die Religiosität des Patienten (Tedeschi und Calhoun 1996). Neben Alter, Geschlecht, Familienstand und Religion sollte man zusätzlich den Bildungsstand und die soziale Unterstützung inkludieren. Eine getrennte Analyse der sozialen Unterstützung als Einflussfaktor ist deshalb sinnvoll, da der Familienstand diesen Parameter nur in Teilen abdeckt. Das soziale Umfeld wird deshalb als potenzieller Einflussfaktor der PTR gewertet, da Patienten mit festen sozialen Bindungen eher die Möglichkeit zu einem interpersonellen Austausch besitzen. Sie können sich engen Vertrauten 
öffnen und mit ihnen über ihre Gedanken und Ängste sprechen (Swickert und Hittner 2009). Es wird davon ausgegangen, dass der Bewältigungsprozess durch die erfahrene Unterstützung erleichtert wird und die Betroffenen dadurch resilienter sind (Lepore und Revenson 2006). Der als besonders wichtig erachtete Faktor „Soziale Unterstützung“ kommt im PPR-Fragebogen nicht vor und war somit nicht Teil der untersuchten Einflussfaktoren in der vorliegenden Studie. In den Freitexten betonten die Patienten jedoch, dass sie die fehlende Kommunikation mit den Ärzten in starkem Maße belastet und sie dankbar für die Zuwendung von Familie und Freunden sind.

Neben der Datenerhebung resultierten auch Schwierigkeiten bei der Validierung von Ergebnissen mittels Fragebögen. Ein möglicherweise auftretendes Problem ist die Variabilität der Interpretation der Fragen. Die Verbesserung der interpersonellen Beziehungen wird möglicherweise nicht von allen Patienten als Gewinn wahrgenommen (Tedeschi und Calhoun 1996). Wortman (2004) stellt daher die Ergebnisse der empirischen Studien von Tedeschi und Calhoun (2004) infrage. Zu Recht weist sie auf die Fehlerquelle hin, die sich durch die unterschiedlichen Interpretationen des Wortes „Wachstum“ ergibt. Wenn Personen angeben, dass ein Ereignis sie stärker gemacht hat, ist es fraglich, ob dies als Nennwert für Wachstum qualifiziert werden kann, da kein allgemein anerkannten Messwert für psychologisches Wachstum existiert (Wortman 2004).

\subsection{Schlussfolgerungen}

$\mathrm{Zu}$ konstatieren bleibt die Tatsache, dass Traumata zwar eine mögliche Ursache für eine subjektiv empfundene Weiterentwicklung sein können, dass das Erleben von Traumata allein aber keine hinreichende Bedingung für eine PTR darstellt. Einige Menschen erfahren posttraumatisch keine persönliche Reifung. Gerade diese Menschen sind auf Maßnahmen zur Verbesserung ihrer Lebensqualität sowie auf professionelle und private Unterstützung in besonderem Maße angewiesen. Bei der PTR handelt sich nicht um einen normativen Wachstumsprozess, der von jedem Traumaüberlebenden erwartet werden kann (Maercker 2009).

Weiter belegen die Daten der vorliegenden Studie, dass eine kombinierte Untersuchung von PTR und Lebensqualität deshalb als sinnvoll erscheint, da der Verdacht der Kohärenz besteht. Allerdings sollten eine Fokussierung auf positive persönliche posttraumatische Entwicklungen und somit eine Pathologisierung abweichender Entwicklungsverläufe vermieden werden. 


\section{ZUSAMMENFASSUNG}

Resümierend lässt sich feststellen, dass schwerwiegende Traumata sowohl negative Effekte, wie eine posttraumatische Belastungsstörung, als auch subjektiv empfundene positive Effekte, wie eine Posttraumatische Reifung (PTR), auslösen können.

Ziel der retrospektiven Analyse war die Erforschung der PTR bei Patienten mit Plattenepithelkarzinomen im Kopf-Hals-Bereich.

Dazu gehörten die posttraumatische Verlaufsbetrachtung der PTR und der Lebensqualität, die Analyse potenzieller sozio-demografischer Einflussfaktoren sowie der mögliche Zusammenhang zwischen PTR und Lebensqualität.

Bei der Studie handelt es sich um eine retrospektive Analyse der vorliegenden Fragebögen. Die Erfassung der PTR erfolgte über den PPR-Fragebogen von Tedeschi und Calhoun. Die Lebensqualität wurde mit der deutschen Version des UW QOL-Fragebogens V4 der Universität Washington bestimmt. Zur Auswertung konnten lediglich $50 \%$ der vorliegenden Daten, also $n=15$ Patienten, mit einem primären Plattenepithelkarzinom im Kopf-HalsBereich, die im Zeitraum von 2011 bis 2012 im Universitätsklinikum Göttingen therapiert wurden, verwendet werden. Die Auswertung der PPR-Fragebögen ergab eine PTR, die am stärksten unmittelbar nach dem Trauma (maximal 30 Tage) wahrgenommen wurde und im Laufe des Untersuchungszeitraums (360 Tage) kontinuierlich und signifikant abnahm. Es ließ sich ein deutlicher Zusammenhang zwischen der PTR und dem Faktor Alter feststellen.

Jüngere Patienten beschrieben eine gesteigerte Wahrnehmung „Neuer Möglichkeiten“ $(p<0,05)$. „Persönliche Beziehungen“" wurden verstärkt von Atheisten erlebt $(p<0,05)$, und hinsichtlich des weiblichen Geschlechts und dem Erleben „Neuer Möglichkeiten“ ließ sich ein tendenzieller Zusammenhang erkennen $(\mathrm{p}=0,07)$. Hinsichtlich der Lebensqualität ist die Verschlechterung von prä- zu postoperativ aufgefallen und ist in den einzelnen Verläufen detailliert dargestellt worden. Zur besseren Übersicht und Validierung wurde die Aufteilung der Lebensqualität in die physische und die sozial-emotionale Funktion übernommen. Bei allen Patienten verschlechterten sich postoperativ sowohl die physische als auch die sozial-emotionale Funktion der Lebensqualität.

Die soziodemografischen Faktoren Alter, Religion und Familienstand nahmen keinen signifikanten Einfluss auf die Lebensqualität. Der Parameter Geschlecht verfehlte die Signifikanzgrenze nur ganz knapp, was auf einen potenziellen Einfluss hindeutet $(\mathrm{p}=0,058)$. Bei der Überprüfung des Zusammenhangs zwischen Lebensqualität und PTR ließ sich eine negative Korrelation feststellen, gleichbedeutend damit, dass Patienten mit einer schlech- 
ten $Q O L$ eine geringere PTR erleben. Eine bessere $Q O L$ zeigte einen signifikanten Zusammenhang mit „Neue Möglichkeiten“, „Wertschätzung des Lebens“ und „Persönliche Stärke“ $(\mathrm{p}<0,05)$.

Weitere Studien mit größeren Patientenkollektiven sind deshalb unumgänglich, um den zeitlichen Verlauf und die Einflussfaktoren für eine PTR gründlicher überprüfen zu können. Die Testung der Korrelation von $Q O L$ und PTR erscheint sinnvoll und kann durch ein größeres und einheitlicheres Patientenkollektiv zu aussagekräftigeren Ergebnissen führen. 


\section{ANLAGE}

\subsection{Tabellen}

Tabelle 15: MW und SD der physischen Funktionen nach Zeitpunkt

\begin{tabular}{|c|c|c|c|c|c|c|c|c|}
\hline & & & Kauen & Schlucken & Aussehen & Speichel & Sprache & Geschmack \\
\hline \multirow[t]{21}{*}{ Zeitpunkt } & prä-op & Mittelwert & 16 & 10,7 & 15,0 & 13,3 & 8,7 & 2,0 \\
\hline & & Standardabweichung & 24,4 & 20,5 & 22,8 & 30,6 & 20,0 & 7,7 \\
\hline & & Gültige $\mathrm{N}$ & 15 & 15 & 15 & 15 & 15 & 15 \\
\hline & post-op & Mittelwert & 43,3 & 30,7 & 30,0 & 15,3 & 18,7 & 13,3 \\
\hline & & Standardabweichung & 32,0 & 32,8 & 23,5 & 25,3 & 20,7 & 25,3 \\
\hline & & Gültige N & 15 & 15 & 15 & 15 & 15 & 15 \\
\hline & 30 Tage & Mittelwert & 36,7 & 26,0 & 23,3 & 17,3 & 21,3 & 17,3 \\
\hline & & Standardabweichung & 29,7 & 26,7 & 20,0 & 25,2 & 24,5 & 25,2 \\
\hline & & Gültige $N$ & 15 & 15 & 15 & 15 & 15 & 15 \\
\hline & 90 Tage & Mittelwert & 30,0 & 28,0 & 23,3 & 24,0 & 22,7 & 19,3 \\
\hline & & Standardabweichung & 25,4 & 25,7 & 20,0 & 27,5 & 19,4 & 24,9 \\
\hline & & Gültige N & 15 & 15 & 15 & 15 & 15 & 15 \\
\hline & 180 Tage & Mittelwert & 33,3 & 28,0 & 25,0 & 28,7 & 22,7 & 21,3 \\
\hline & & Standardabweichung & 24,4 & 25,7 & 23,1 & 29,0 & 19,4 & 24,5 \\
\hline & & Gültige N & 15 & 15 & 15 & 15 & 15 & 15 \\
\hline & 270 Tage & Mittelwert & 33,3 & 25,3 & 25,0 & 25,3 & 18,7 & 15,3 \\
\hline & & Standardabweichung & 24,4 & 22,9 & 25,0 & 22,9 & 20,7 & 25,3 \\
\hline & & Gültige N & 15 & 15 & 15 & 15 & 15 & 15 \\
\hline & 360 Tage & Mittelwert & 30,0 & 25,3 & 23,3 & 20,7 & 20,7 & 17,3 \\
\hline & & Standardabweichung & 25,4 & 22,9 & 25,8 & 20,2 & 20,2 & 25,2 \\
\hline & & Gültige $\mathrm{N}$ & 15 & 15 & 15 & 15 & 15 & 15 \\
\hline
\end{tabular}

Tabelle 16: MW und SD der sozial-emotionalen Funktionen nach Zeitpunkt

\begin{tabular}{|c|c|c|c|c|c|c|c|c|}
\hline & & & Stimmung & Angst & Erholung & Aktivität & Schmerz & Schulter \\
\hline \multirow{21}{*}{ Zeitpunkt } & 180 Tage & Mittelwert & 30,0 & 25,3 & 26,7 & 26,7 & 16,7 & 20,0 \\
\hline & & Standardabweichung & 21,5 & 22,9 & 33,4 & 25,8 & 15,4 & 28,5 \\
\hline & & Gültige N & 15 & 15 & 15 & 15 & 15 & 15 \\
\hline & 270 Tage & Mittelwert & 33,3 & 29,3 & 33,3 & 26,7 & 18,3 & 13,3 \\
\hline & & Standardabweichung & 26,2 & 26,9 & 32,3 & 29,1 & 22,1 & 25,3 \\
\hline & & Gültige N & 15 & 15 & 15 & 15 & 15 & 15 \\
\hline & 30 Tage & Mittelwert & 33,3 & 28,0 & 25,0 & 26,7 & 20,0 & 22,0 \\
\hline & & Standardabweichung & 24,4 & 25,7 & 25,0 & 20,0 & 16,9 & 28,1 \\
\hline & & Gültige $N$ & 15 & 15 & 15 & 15 & 15 & 15 \\
\hline & 360 Tage & Mittelwert & 28,3 & 29,3 & 26,7 & 21,7 & 13,3 & 13,3 \\
\hline & & Standardabweichung & 22,9 & 26,9 & 33,4 & 29,7 & 22,9 & 25,3 \\
\hline & & Gültige N & 15 & 15 & 15 & 15 & 15 & 15 \\
\hline & 90 Tage & Mittelwert & 35,0 & 26,0 & 30,0 & 30,0 & 20,0 & 20,0 \\
\hline & & Standardabweichung & 24,6 & 26,7 & 30,2 & 28,7 & 14,0 & 28,5 \\
\hline & & Gültige N & 15 & 15 & 15 & 15 & 15 & 15 \\
\hline & post-op & Mittelwert & 36,7 & 30,0 & 26,7 & 33,3 & 23,3 & 17,3 \\
\hline & & Standardabweichung & 29,7 & 30,0 & 29,1 & 22,5 & 20,0 & 25,2 \\
\hline & & Gültige N & 15 & 15 & 15 & 15 & 15 & 15 \\
\hline & prä-op & Mittelwert & 30,0 & 34,7 & 21,7 & 16,7 & 23,3 & ,0 \\
\hline & & Standardabweichung & 30,2 & 35,0 & 32,6 & 20,4 & 17,6 & ,0 \\
\hline & & Gültige N & 15 & 15 & 15 & 15 & 15 & 15 \\
\hline
\end{tabular}




\section{LITERATURVERZEICHNIS}

Almedom A (2005): Resilience, Hardiness, Sence of coherence, and posttraumatic growth: all paths leading to "light at the end of the tunnel"? J Loss and Trauma 10, 253-265

Altieri A, Bosetti C, Talamini S, Gallus S, Franceschi S, Levi F, Dal Maso L, Negri E, La Vecchia C (2002): Cessation of smoking and drinking and the risk of laryngeal cancer. Br J Cancer 87, 1227-1229

Antonovsky A: Salutogenese. Zur Entmystifizierung der Gesundheit. (Forum für Verhaltenstherapie und psychosoziale Praxis, Band 36) Übersetzt und hrsg. v. Franke A. dgvtVerlag, Tübingen 1997

Biazevic MGH, Antunes JLF, Togni J, de Andrade FP, de Carvalho MB, Wünsch-Filho V (2010): Survival and quality of life of patients with oral and oropharyngeal cancer at 1year follow-up of tumor resection. J Appl Oral Sci $\underline{18}$, 279-284

Borggreven PA, Verdonck-de Leeuw IM, Muller JM, Heiligers MLCH, de Bree R, Aaronson NK, Leemans CR (2007): Quality of life and functional status in patients with cancer of the oral cavity and oropharynx: pretreatment values of a prospective study. Eur Arch Otorhinolaryngol 264, 651-657

Bose P, Brockton NT, Dort JC (2013): Head and neck cancer: From anatomy to biology. Int J Cancer 133, 2013-2023

Bower JE, Meyerowitz BE, Desmond KA, Bernaards CA, Rowland JH, Ganz PA (2005): Perceptions of positive meaning and vulnerability following breast cancer: predictors and outcomes among long-term breast cancer survivors. Ann Behav Med 29, 236-245

Brewin CR, Andrews B, Valentine JD (2000): Meta-analysis of risk factors for posttraumatic stress disorder in trauma-exposed adults. J Consult Clin Psychol $\underline{68}$, 748-766

Brierley J, Gospodarowicz MK, Wittekind C (Hrsg.): TNM Classification of malignant tumors. 8. Auflage. Wiley Blackwell/John Wiley \& Sons, Chichester (UK), Hoboken (NJ) 2017

Brunet J, McDonough MH, Hadd V, Crocker PRE, Sabiston CM (2010): The Posttraumatic Growth Inventory: An examination of the factor structure and invariance among breast cancer survivors. Psychooncology $\underline{19}, 830-838$

Calhoun LG, Tedeschi RG: The foundations of posttraumatic growth: an expanded framework. In: Calhoun LG, Tedeshi RG (Hrsg.): Handbook of posttraumatic growth. Research and practice. Lawrence Erlbaum Associates, Mahwah (NJ) 2006, 1-23

Calhoun LG, Cann A, Tedeschi RG, McMillan J (2000): A correlational test of the relationship between posttraumatic growth, religion and cognitive processing. J Trauma Stress $\underline{13}, 521-527$

Cann A, Calhoun LG, Tedeschi RG, Kilmer RP, Gil-Rivas V, Vishnevsky T, Danhauer SC (2010): The Core Beliefs Inventory: a brief measure of disruption in the assumptive world. Anxiety Stress Coping 23, 19-34 
Collins RL, Taylor SE, Skokan LA (1990): A Better World or a Shattered Vision? In: Changes in Life Perspectives Following Victimization. Soc Cogn $\underline{8}$, 263-285

Connerty TJ, Knott V (2013): Promoting positive change in the face of adversity: experiences of cancer and post-traumatic growth. Eur J Cancer Care (Engl) 22, 334-344

Cordova MJ, Cunningham LLC, Carlson CR, Andrykowski MA (2001a): Social Constraints, Cognitive Processing, and Adjustment to Breast Cancer. J Consult Clin Psychol $\underline{69}, 706-711$

Cordova MJ, Lauren L, Cunningham C, Carlson CR, Andrykowski MA (2001b): Posttraumatic growth following breast cancer: A controlled comparison study. Health Psychol 20, 176-185

Ehrenfeld M, Prein J: Tumoren im Mund-Kiefer-Gesichtsbereich. In: Schwenzer N, Ehrenfeld M(Hrsg.): Spezielle Chirurgie. Lehrbuch zur Aus- und Weiterbildung. Georg Thieme, Stuttgart, New York 2002, 99-118

Fooken I: Resilienz und posttraumatische Reifung. In: Maercker A (Hrsg.): Posttraumatische Belastungsstörungen. 3. Auflage, Springer, Berlin Heidelberg 2009, 65-79

Frerich B (2010): Standardtherapie von Plattenepithelkarzinomen der Mundhöhle. Der Onkologe $\underline{16}, 527-538$

Fromm K, Andrykowski MA, Hunt J (1996): Positive and negative psychosocial sequelae of bone marrow transplantation: implications for quality of life assessment. J Behav Med $\underline{19}, 221-240$

Garden AS (2004): Preliminary Results of Radiation Therapy Oncology Group 97-03: A Randomized Phase II Trial of Concurrent Radiation and Chemotherapy for Advanced Squamous Cell Carcinomas of the Head and Neck. J Clin Oncol 22, 2856-2864

Helgeson VS, Reynolds KA, Tomich PL (2006): A meta-analytic review of benefit finding and growth. J Consult Clin Psychol 74, 797-816

ICD-10-GM Systematisches Verzeichnis 2018. Internationale statistische Klassifikation der Krankheiten und verwandter Gesundheitsprobleme. 10. Revision- German Modifikation. Bearb. V. Graubner B. Deutscher Ärzte-Verlag, Köln 2017

Janoff-Bulman R (2004): Posttraumatic Growth: Three Explanatory Models. Psychol Inquiry $\underline{15}, 30-34$

Klauer T (1998): "Still Stable after all This . . .?" Temporal Comparison in Coping with Severe and Chronic Disease. Int J Behav Dev 22, 339-355

Lang S, Wollenberg B, Dellian M, Steuer-Vogt MK, Schwenzer K, Sautier W, Chucholowski M, Eckel R, Faas I, Wilmes E, Ehrenfeld E, Arnold W, Kastenbauer E, Hölzel D (2002): Klinische und epidemiologische Daten zu Malignomen des Kopf-Hals-Bereichs. In: Laryngo-Rhino-Otologie $\underline{81}, 499-508$

Lazarus RS (2006): Emotions and Interpersonal Relationships: Toward a Person-Centered Conceptualization of Emotions and Coping. J Pers $\underline{74}$, 9-46 
Lechner SC, Carver CS, Antoni MH, Weaver KE, Phillips KM (2006): Curvilinear associations between benefit finding and psychosocial adjustment to breast cancer. J Consult Clin Psychol 74, 828-840

Lechner SC, Zakowski SG, Antoni MH, Greenhawt M, Block K, Block P (2003): Do sociodemographic and disease-related variables influence benefit-finding in cancer patients? Psychooncology 12, 491-499

Lefebvre JL, Coche-Dequeant B, Buisset E, Mirabel X, Van JT, Prevost B (1994): Management of early oral cavity cancer. Experience of Centre Oscar Lambret. Eur J Cancer B Oral Oncol $\underline{30}, 216-220$

Leitlinie Mundhöhlenkarzinom s. S3-Leitlinie 2.0. 2012

Lepore SJ (1997): Expressive writing moderates the relation between intrusive thoughts and depressive symptoms. J Pers Soc Psychol 73, 1030-1037

Lepore S, Revenson T: Relationship between posttraumatic growth and resilience: recovery, resistance and reconfiguration. In: Calhoun LG, Tedeschi RG (Hrsg.): Handbook of posttraumatic growth. Research and practice. Lawrence Erlbaum Associates, Mahwah (NJ) 2006, 24-46

Lepore SJ, Silver RC, Wortman CB, Wayment HA (1996): Social constraints, intrusive thoughts, and depressive symptoms among bereaved mothers. J Pers Soc Psychol $\underline{70}$, 271-282

Linley PA, Joseph S (2004): Positive change following trauma and adversity: a review. J Trauma Stress $\underline{17}, 11-21$

Maercker A: Symptomatik, Klassifikation und Epidemiologie. In: Maercker A (Hrsg.): Posttraumatische Belastungsstörungen. 3. Auflage, Springer, Berlin Heidelberg 2009, $14,33-38$

Maercker A, Langner R (2001): Persönliche Reifung (Personal Growth) durch Belastungen und Traumata. Diagnostica $\underline{47}, 153-162$

Maschmeyer G, Koch K, Jungehülsing M (2011): Kopf-Hals-Tumoren. Best practice onkologie $\underline{6}, 4-20$

Memtsa PT, Tolia M, Tzitzikas I, Bizakis J, Pistevou-Gombaki K, Charalambidou M, Kyrgias G (2017): Assessment of xerostomia and its impact on quality of life in head and neck cancer patients undergoing radiation therapy. Mol Clin Oncol $\underline{6}, 789-793$

Mohr C, Bohndorf W, Carstens J, Harle F, Hausamen JE, Hirche H, Kimmig H, Kutzner J, Muhling J, Reuther J (1994): Preoperative radiochemotherapy and radical surgery in comparison with radical surgery alone. A prospective, multicentric, randomized DOSAK study of advanced squamous cell carcinoma of the oral cavity and the oropharynx (a 3year follow-up). Int J Oral Maxillofac Surg 23, 140-148

Rabbels J, Wyzisk M, Siessegger M, Klesper B, Reuther T, Kubler AC (2005): Quality of life of patients with squamous cell carcinoma of the oral cavity. Mund Kiefer Gesichtschir $\underline{9}, 300-305$ 
Reichart PA, Hausamen JE, Becker J, Neukam FW, Schliephake H, Schmelzeisen R: Curriculum Zahn-, Mund- und Kieferkrankheiten, Chirurgie Band II, Quintessenz Verlags$\mathrm{GmbH}$, Berlin 2002, 419-420.

Rogers SN, Lowe D, Yueh B, Weymuller EA (2010): The physical function and socialemotional function subscales of the University of Washington Quality of Life Questionnaire. Arch Otolaryngol Head Neck Surg 136, 352-357

Rogers SN, Humphris G, Lowe D, Brown JS, Vaughan ED (1998): The impact of surgery for oral cancer on quality of life as measured by the Medical Outcomes Short Form 36. Oral Oncol 34, 171-179

Schliephake H, Jamil MU (2002): Prospective evaluation of quality of life after oncologic surgery for oral cancer. Int J Oral Maxillofac Surg 31, 427-433

Schmidt SD, Blank TO, Bellizzi KM, Park CL (2012): The relationship of coping strategies, social support, and attachment style with posttraumatic growth in cancer survivors. J Health Psychol 17, 1033-1040

Schreiber JA, Brockopp DY (2012): Twenty-five years later-what do we know about religion/spirituality and psychological well-being among breast cancer survivors? A systematic review. J Cancer Surviv $\underline{6}, 82-94$

Sears R, Stanton AL, Danoff-Burg S (2003): The yellow brick road and the emerald city: Benefit finding, positive reappraisal coping and posttraumatic growth in women with early-stage breast cancer. Health Psychol 22, 487-497

Shakespeare W: As you like it. Englisch-deutsche Studienausgabe. Dt. Prosafassung und Anm. von Ilse Leisi unter Mitw. Von Hugo Schwaller. Stauffenburg-Verl., Tübingen 2000

Swickert R, Hittner J (2009): Social Support, Coping Mediates, the Relationship between Gender and Posttraumatic Growth. J Health Psychol 14, 387-393

S3-Leitlinie Mundhöhlenkarzinom: Diagnostik und Therapie des Mundhöhlenkarzinoms. Version 2.0. 2012

Thurnher D, Heiduschka G (2011): Prävention bei Kopf- und Halskarzinomen? Ein Überblick. sportmed präventivmed $\underline{41}(3), 6-7$

Tedeschi RG, Calhoun LG (1996): The Posttraumatic Growth Inventory: Measuring the positive legacy of trauma. J Trauma Stress $\underline{13}, 455-471$

Tedeschi RG, Calhoun LG (2004): Posttraumatic Growth: Conceptual Foundations and Empirical Evidence. Psychol Inq 15, 1-18

Tempelaar R, de Haes JC, de Ruiter JH, Bakker D, van Den Heuvel WJ, van Nieuwenhuijzen MG (1989): The social experiences of cancer patients under treatment: a comparative study. Soc Sci Med 29, 635-642

Tomich PL, Helgeson VS (2004): Is finding something good in the bad always good? Benefit finding among women with breast cancer. Health Psychol 23, 16-23 
Tomich PL, Helgeson VS (2012): Posttraumatic growth following cancer: links to quality of life. J Trauma Stress 25, 567-573

Wicks L, Mitchell A (2010): The adolescent cancer experience: loss of control and benefit finding. Eur J Cancer Care (Engl) 19, 778-78

Widows MR, Jacobsen PB, Booth-Jones M, Fields KK (2005): Predictors of posttraumatic growth following bone marrow transplantation for cancer. Health Psychol 24, 266-273

Wittekind C, Weber A, Weidenbach H (2001): Pathologie und Prognosefaktoren von Plattenepithelkarzinomen des Kopf-Hals-Bereiches. Onkologe 7, 498-504

Wortman CB (2004): Posttraumatic Growth: Progress and Problems. Psychol Inq $\underline{15}$, 8190

Villaret AB, Cappiello J, Piazza C, Pedruzzi B, Nicolai P (2008): Quality of life in patients treated for cancer of the oral cavity requiring reconstruction: a prospective study. Acta Otorhinolaryngol Ital $\underline{28}, 120-125$

Zoellner T, Maercker A (2006): Posttraumatic growth in clinical psychology - a critical review and introduction of a two component model. Clin Psychol Rev 26, 626-653

Zoellner T, Calhoun LG, Tedeschi RG: Trauma und persönliches Wachstum. In: Maercker A, Rosner R (Hrsg.): Psychotherapie der posttraumatischen Belastungsstörungen, 1. Auflage; Georg Thieme Verlag, Stuttgart 2006, 37-38

Zoellner T, Rabe S, Karl A, Maercker A (2008): Posttraumatic growth in accident survivors: openness and optimism as predictors of its constructive or illusory sides. J Clin Psychol $\underline{64}, 120$ 


\section{DANKSAGUNG}

Mein herzlichstes Dankeschön geht an

- Herrn PD Dr. med. Dr. med. dent. Gruber für die Überlassung des Themas der vorliegenden Dissertation und die sehr gute Betreuung im Rahmen meiner Dissertation.

- David Ellenberger aus dem Institut für statistische Betreuung für die wundervolle statistische Beratung und Unterstützung. 


\section{LEBENSLAUF}

\section{Johanna Leonhard}

Am 17.02.1987 wurde ich als dritte Tochter von Hildegard Leonhard (geborene Wulf) und Dr. Dieter Leonhard in Lauterbach geboren. Von 1993 bis 1997 besuchte ich die EichbergGrundschule in Lauterbach. Die allgemeine Hochschulreife erlangte ich 2006 am Marianum in Fulda. Im Wintersemester 2006 begann ich in Würzburg das Studium für gymnasiales Lehramt in den Fächern Chemie und Physik. Während dieser Zeit verstärkte sich bei mir der Gedanke, ein Zahnmedizinstudium zu beginnen. Daraufhin wechselte ich zum Wintersemester 2007 nach Göttingen und begann mein Zahnmedizinstudium. Das Staatsexamen schloss ich im Jahr 2012 erfolgreich ab. Kurz darauf begann ich meine Assistenzzeit in einer zahnärztlichen Praxis in Berlin. Meine Assistenzzeit beendete ich im Juli 2014 und arbeite seitdem als angestellte Zahnärztin in Berlin. 
